\title{
Electroweak Measurements using Heavy Quarks at LEP
}

\author{
T. Behnke \\ Universität Hamburg/DESY, II Institut für Experimental Physik, Notkestrasse 85, \\ D-22607 Hamburg, Germany
}

\section{D.G. Charlton}

Royal Society University Research Fellow,

School of Physics and Space Research, University of Birmingham, Birmingham B15 2TT, United Kingdom, and

PPE Division, CERN, CH-1211 Geneva 23, Switzerland

\begin{abstract}
Electroweak measurements at the LEP electron-positron collider have been made both for different lepton and quark flavours. The measurements in the heavy quark sector, specifically of the partial widths for $Z^{0}$ decays to $b \bar{b}$ and $c \bar{c}$ and the forward-backward $\mathrm{b}$ and $\mathrm{c}$ quark production asymmetries, are reviewed. Combined values from the LEP measurements are derived of $\Gamma_{\mathrm{b}} / \Gamma_{\text {had }}=0.2207 \pm 0.0022, \Gamma_{\mathrm{c} \overline{\mathrm{c}}} / \Gamma_{\mathrm{had}}=0.153 \pm 0.011$, and for the asymmetries at $\sqrt{s}=91.26 \mathrm{GeV}, A_{\mathrm{FB}}^{\mathrm{b}}=0.0902 \pm 0.0045$ and $A_{\mathrm{FB}}^{\mathrm{c}}=0.070 \pm 0.012$. In the framework of the standard model, a value of $\Gamma_{\mathrm{b}} / \Gamma_{\text {had }}=0.2189 \pm 0.0020$ is obtained (for $\Gamma_{\mathrm{c} \overline{\mathrm{c}}} / \Gamma_{\mathrm{had}}=0.171$ ). The $\mathrm{b}$ asymmetry is used to measure the effective electroweak mixing angle $\sin ^{2} \theta_{\mathrm{W}}^{\text {eff }}=0.2328 \pm 0.0008$.
\end{abstract}




\section{Introduction}

The LEP electron-positron collider at CERN started operation in 1989 with the goal of studying the properties of the intermediate vector boson of the weak neutral current, the $\mathrm{Z}^{0}$. Precise measurements have been made of the $\mathrm{Z}^{0}$ mass, its width and leptonic couplings using leptonic and inclusive hadronic decays of the $\mathrm{Z}^{0}[1]$. With the large statistics now available from the first few years of LEP operation, the reach of high precision physics has been extended into the investigation of heavy quarks. In particular the couplings of individual quark flavours have become the subject of increasing interest. They are measured via the partial width for a $\mathrm{Z}^{0}$ decay into a pair of quarks of a specific flavour, and from forward-backward production asymmetries.

In this paper the current status of these measurements is reviewed. The motivation for the investigations are discussed in the next section, followed by a detailed presentation of the techniques currently in use. Particular emphasis is given in section 3 to methods of preparing samples of bottom, b, and charm, c, quarks. The results obtained by the LEP experiments are reviewed in sections 4-6, and combined and compared with standard model predictions in section 7. Measurements of light quark electroweak observables are relatively limited, but are briefly reviewed in section 8 . Future prospects for the heavy quark measurements are discussed in section 9 .

\section{Motivations}

One of the principal physics goals of the LEP collider is to measure accurately the couplings of the $\mathrm{Z}^{0}$ to quarks and leptons. Measurements of the $\mathrm{Z}^{0}$ cross-section as a function of energy, the lineshape, the total hadronic decay width, $\Gamma_{\text {had }}$, the leptonic partial widths, the forwardbackward asymmetries of decays to leptons, and of the polarisation of $\tau$ leptons produced in $\mathrm{Z}^{0}$ decays, have all contributed to the current extremely precise knowledge of the couplings of the $\mathrm{Z}^{0}$ to each flavour of lepton. In the quark sector measurements are harder to make because of the difficulty in distinguishing between the different primary quark flavours produced in $\mathrm{Z}^{0}$ decays. Heavy quarks, however, provide a powerful insight into this sector, because their large masses mean that they are only rarely produced in the hadronisation process, rather than directly from $\mathrm{Z}^{0}$ decay. The large masses and detectable lifetimes of hadrons containing heavy quarks further mean that it is possible to separate events containing them from light-quark backgrounds.

At LEP, the neutral current couplings of heavy quarks can be probed by measuring the partial $\mathrm{Z}^{0}$ decay widths to $\mathrm{b} \overline{\mathrm{b}}$ and $c \overline{\mathrm{c}}$ (denoted $\Gamma_{\mathrm{b} \overline{\mathrm{b}}}$ and $\Gamma_{\mathbf{c} \overline{\mathbf{c}}}$ respectively), and the angular distribution of the produced heavy quarks relative to the beam axis. These quantities are predicted by the standard model of the electroweak interaction. However, since only five of the six quark flavours are experimentally well established, and since no direct evidence for the Higgs boson exists as yet, numerical predictions can only be made as a function of the unknown masses of the top quark and the Higgs boson, denoted $m_{\text {top }}$ and $m_{\text {Higgs }}$, respectively. Most electroweak observables depend on these parameters through radiative corrections involving a virtual top quark or Higgs boson.

In the lowest-order Born approximation, the partial $\mathrm{Z}^{0}$ decay width to a fermion-antifermion 
pair $f \bar{f}, \Gamma_{f \bar{f}}$, is expressed as a function of the coupling constants:

$$
\Gamma_{\mathrm{f} \overline{\mathrm{f}}}=\frac{G_{\mu} m_{\mathrm{Z}}^{3}}{2 \pi \sqrt{2}}\left(\beta \frac{3-\beta^{2}}{2}\left(g_{\mathrm{V}}^{\mathrm{f}}\right)^{2}+\beta^{3}\left(g_{\mathrm{A}}^{\mathrm{f}}\right)^{2}\right) .
$$

Here $\beta$ is the velocity of the produced fermion $\mathrm{f}$ in the $\mathrm{Z}^{0}$ rest frame, $G_{\mu}$ the Fermi decay constant, $m_{\mathrm{Z}}$ the $\mathrm{Z}^{0}$ mass, and $g_{\mathrm{V}}^{\mathrm{f}}, g_{\mathrm{A}}^{\mathrm{f}}$ the vector and axial-vector electroweak neutral current coupling constants. These latter are expressible:

$$
\begin{aligned}
g_{\mathrm{V}}^{\mathrm{f}} & =I_{f}^{3, L}-2 Q^{\mathrm{f}} \sin ^{2} \theta_{\mathrm{W}} \\
g_{\mathrm{A}}^{\mathrm{f}} & =I_{f}^{3, L},
\end{aligned}
$$

where $I_{f}^{3, L}$ is the third component of weak isospin, taking values $I_{f}^{3, L}=\frac{1}{2},-\frac{1}{2}$, for $\mathrm{c}$ and b quarks, respectively, and $Q^{\mathrm{f}}$ is the fermion charge, $Q^{\mathrm{f}}=\frac{2}{3},-\frac{1}{3}$, for $\mathrm{c}$ and $\mathrm{b}$. The quantity $\sin ^{2} \theta_{\mathrm{W}}$, the electroweak mixing angle, is defined by

$$
\sin ^{2} \theta_{\mathrm{W}} \equiv 1-m_{\mathrm{W}}^{2} / m_{\mathrm{Z}}^{2} .
$$

This lowest-order formalism is, however, insufficiently accurate for precise electroweak measurements made at LEP: the radiative corrections have to be taken into account.

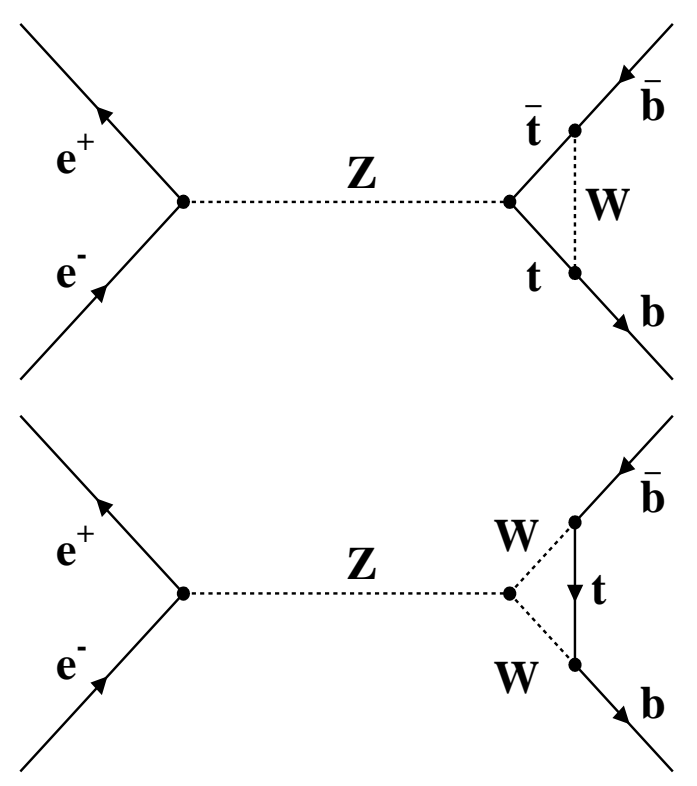

Figure 1: Two Feynman diagrams which contribute to the $\mathrm{Z}^{0}-\mathrm{b} \overline{\mathrm{b}}$-specific vertex corrections. Such diagrams are unimportant for final $d \bar{d}$ and $s \bar{s}$ flavours because the $d$ and $s$ quarks lie in different weak isospin doublets to the top quark.

The $\mathrm{Z}^{0}-\mathrm{b} \overline{\mathrm{b}}$ vertex is particularly interesting in this context. Radiative corrections affect this vertex differently to those of other lighter fermion flavours because the $b$ quark is in the same weak isospin doublet as the top quark. This means that loop diagrams involving top quarks, such as those shown in figure 1, contribute quite differently to this vertex than to the others. The other important graphs in this context are corrections to the $\mathrm{Z}^{0}$ propagator, which affect all 
five flavours equally. The top mass dependent propagator corrections happen largely to cancel the vertex corrections for b-quarks, and thus $\Gamma_{\mathrm{b}}$ is rather insensitive to the top quark mass. The total hadronic width $\Gamma_{\text {had }}$, on the other hand, depends on $m_{\mathrm{t}}$, so that the experimentally measurable ratio $\Gamma_{\mathrm{b}} / \Gamma_{\text {had }}$ is rather more sensitive to the value of the top quark mass than the corresponding ratio for other fermions. For all flavours this quantity is rather insensitive to the Higgs mass. This is illustrated in figure 2. The sizeable top mass dependence combined with a
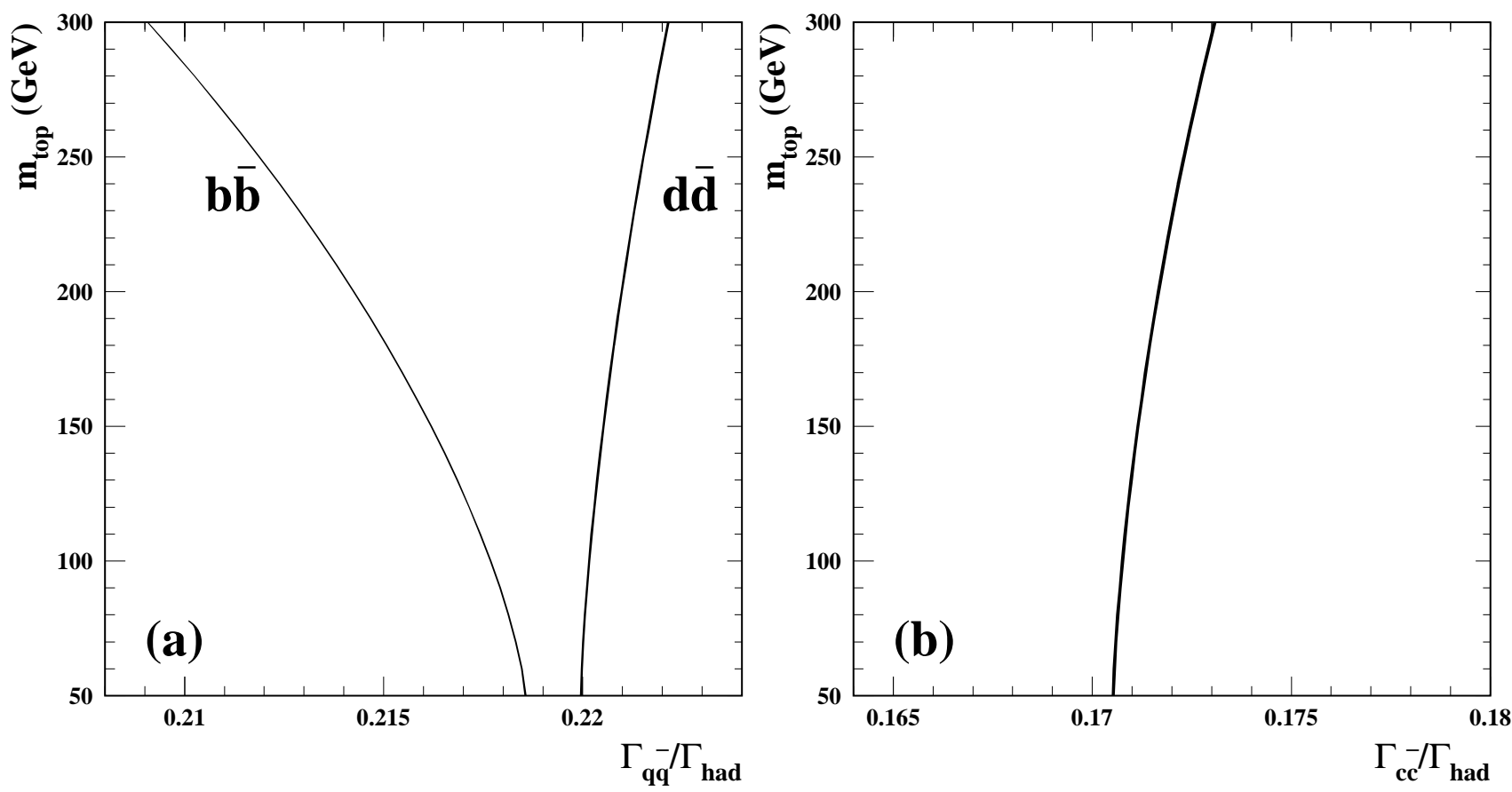

Figure 2: The standard model predictions, taken from ZFITTER [2], for the variation of (a) $\Gamma_{\mathrm{b}} / \Gamma_{\text {had }}$ and $\Gamma_{\mathrm{d}} / \Gamma_{\mathrm{d} a d}$, and (b) $\Gamma_{\mathrm{c} \bar{c}} / \Gamma_{\text {had }}$, with $m_{\mathrm{top}}$. The top mass variation of $\Gamma_{\mathrm{b}} / \Gamma_{\mathrm{had}}$ is greatest. The width of the curves indicates the variation obtained by varying the mass of the Higgs particle between 60 and $1000 \mathrm{GeV}$.

small Higgs mass dependence makes $\Gamma_{\mathrm{b}} / \Gamma_{\text {had }}$ rather special among the electroweak observables at $\mathrm{Z}^{0}$ energies. Other electroweak observables have rather more sensitivity to the top mass [3], but the Higgs mass dependence of them is also large. The top mass dependences of $\Gamma_{\mathrm{b}} / \Gamma_{\text {had }}$ and other electroweak measurements have been used together to put indirect limits on the top quark mass from LEP data $[1,3]$. The constraint resulting from measurements of $\Gamma_{\mathrm{b}} / \Gamma_{\text {had }}$ alone is discussed in section 7 .

The argument can be turned around for the next lighter quark, charm. The partial width ratio $\Gamma_{\mathbf{c} \overline{\mathbf{c}}} / \Gamma_{\text {had }}$ is rather insensitive to the assumed value of the top mass. Equally it is only weakly dependent on the Higgs mass, and so is predicted rather precisely in the standard model, even with today's knowledge. The standard model prediction is illustrated in figure 2(b). A precise measurement of $\Gamma_{\mathbf{c} \overline{\mathbf{c}}} / \Gamma_{\text {had }}$ can therefore be a rather good test of the standard model, independent of the unknown particle masses. Any deviations observed from the standard model predictions are difficult to explain without resorting to rather unorthodox scenarios.

Another way of directly accessing information about the coupling constants in the electroweak sector is the investigation of the angular distribution of the final state fermions. On 
the pole of the $\mathrm{Z}^{0}$ resonance the vector and the axial-vector components of the neutral current interfere and result in an asymmetry relative to the direction of the initial state fermion. Interference also occurs between the electromagnetic and neutral weak currents. On the peak this interference vanishes, but it becomes more and more important at energies away from the $\mathrm{Z}^{0}$ pole.

The forward-backward asymmetry, $A_{\mathrm{FB}}^{\mathrm{f}}$, for the process $\mathrm{e}^{+} \mathrm{e}^{-} \rightarrow \mathrm{Z}^{0} \rightarrow \mathrm{f} \overline{\mathrm{f}}$, is defined:

$$
A_{\mathrm{FB}}^{\mathrm{f}} \equiv \frac{\sigma_{\mathrm{F}}-\sigma_{\mathrm{B}}}{\sigma_{\mathrm{F}}+\sigma_{\mathrm{B}}}
$$

where $\sigma_{\mathrm{F}} / \sigma_{\mathrm{B}}$ are the cross-sections for the fermion $\mathrm{f}$ to be produced in the forward/backward hemisphere, respectively, and where the forward hemisphere refers to $\cos \theta>0$, where $\theta$ is the polar angle at which the outgoing fermion is produced relative to the $\mathrm{e}^{-}$beam direction. In the standard model to lowest order, for centre-of-mass energies close to the $\mathrm{Z}^{0}$ mass, the distribution in $\cos \theta$ can be written:

$$
\frac{\mathrm{d} \sigma}{\mathrm{d} \cos \theta} \propto 1+\cos ^{2} \theta+\frac{8}{3} A_{\mathrm{FB}}^{\mathrm{f}} \cos \theta
$$

On the pole of the $\mathrm{Z}^{0}$ resonance for heavy quark flavour $\mathrm{Q}, A_{\mathrm{FB}}^{\mathrm{Q}, 0}$, the forward-backward asymmetry, can again be expressed as a function of the electroweak coupling constants $g_{\mathrm{V}}^{\mathrm{f}}$ and $g_{\mathrm{A}}^{\mathrm{f}}$ :

$$
A_{\mathrm{FB}}^{\mathrm{Q}, 0}=\frac{3}{4} \mathcal{A}_{\mathrm{e}} \mathcal{A}_{\mathrm{Q}}
$$

where $\mathcal{A}_{\mathrm{e}}$ and $\mathcal{A}_{\mathrm{Q}}$ are given by:

$$
\mathcal{A}_{\mathrm{f}}=\frac{2 g_{\mathrm{V}}^{\mathrm{f}} g_{\mathrm{A}}^{\mathrm{f}}}{\left(g_{\mathrm{V}}^{\mathrm{f}}\right)^{2}+\left(g_{\mathrm{A}}^{\mathrm{f}}\right)^{2}}=\frac{2\left(1-4\left|Q^{\mathrm{f}}\right| \sin ^{2} \theta_{\mathrm{W}}\right)}{1+\left(1-4\left|Q^{\mathrm{f}}\right| \sin ^{2} \theta_{\mathrm{W}}\right)^{2}} .
$$

Note that heavy quark asymmetry measurements at LEP can only measure the product $\mathcal{A}_{\mathrm{e}} \mathcal{A}_{\mathrm{Q}}$. However, $\mathcal{A}_{\mathrm{Q}}$ can be measured directly if significant longitudinal polarization is present in the electron or positron beams. In this case $\mathcal{A}_{Q}$ can be derived by measuring the left-right forwardbackward asymmetry. This is currently only possible at the SLC electron-positron collider at SLAC. The SLC results are briefly presented in section 7 .

Electroweak radiative corrections modify these formulae only slightly. The corrections are conventionally absorbed by modifying equation (2) to contain an effective mixing angle $\sin ^{2} \theta_{\mathrm{W}}^{\mathrm{eff}}$, instead of the $\sin ^{2} \theta_{\mathrm{W}}$ defined by equation (4).

Measuring the asymmetry on the pole of the $\mathrm{Z}^{0}$ therefore probes quite directly the weak neutral current couplings or, alternatively, $\sin ^{2} \theta_{\mathrm{W}}$. Repeating the asymmetry measurements at energies away from the pole, even within the small centre-of-mass energy range investigated at LEP-I, gives sensitivity to $\gamma-Z^{0}$ interference and tests the combined electroweak prediction of the standard model.

In practice the situation is modified by the effects of photon radiation and photon-exchange diagrams, which mean that the measured $A_{\mathrm{FB}}^{\mathrm{f}}$ differs from the pole asymmetry, $A_{\mathrm{FB}}^{\mathrm{f}, 0}$, that would be obtained with pure on-mass-shell $\mathrm{Z}^{0}$ decays. This and other small corrections needed to correct from the measured to the pole asymmetry are discussed in section 6.4. 
Measurements of the $\mathrm{Z}^{0}$ lineshape together with lower energy neutrino-scattering measurements indicate that $\sin ^{2} \theta_{\mathrm{W}} \approx 0.23$, predicting:

$$
\begin{aligned}
& \mathcal{A}_{\mathrm{e}} \approx 0.14 \\
& \mathcal{A}_{\mathrm{b}} \approx 0.94 ; A_{\mathrm{FB}}^{\mathrm{b}, 0} \approx 0.10 \\
& \mathcal{A}_{\mathrm{c}} \approx 0.67 ; A_{\mathrm{FB}}^{\mathrm{c}, 0} \approx 0.07
\end{aligned}
$$

The different fermion asymmetries have different sensitivities to $\sin ^{2} \theta_{\mathrm{W}}$ :

$$
\begin{aligned}
& \Delta\left(\sin ^{2} \theta_{\mathrm{W}}\right) \approx-\frac{1}{6} \Delta\left(A_{\mathrm{FB}}^{\mathrm{b}, 0}\right) \\
& \Delta\left(\sin ^{2} \theta_{\mathrm{W}}\right) \approx-\frac{1}{4} \Delta\left(A_{\mathrm{FB}}^{\mathrm{c}, 0}\right)
\end{aligned}
$$

These sensitivities are greater than for $\mathrm{Z}^{0}$ decays to charged lepton pairs $\ell^{+} \ell^{-}$, where:

$$
\Delta\left(\sin ^{2} \theta_{\mathrm{W}}\right) \approx-\frac{1}{2} \Delta\left(A_{\mathrm{FB}}^{\ell, 0}\right)
$$

To understand the properties of the data, it is often necessary to use Monte Carlo simulation programs. The JETSET Monte Carlo program [4], tuned to agree with LEP and lower energy data, is usually employed to model the $\mathrm{Z}^{0}$ decay and the hadronisation process. Each of the LEP experiments has its own special detector simulation program through which Monte Carlo events are passed. These detector simulation packages produce event structures similar to those of the real data, so that the same analysis programs can be used for either type of event.

\section{$3 \quad$ Heavy Quark Tagging Techniques}

At LEP, $\mathrm{Z}^{0}$ 's are produced essentially at rest in the four detectors, ALEPH, DELPHI, L3 and OPAL. All four have a similar construction, with charged particle tracking chambers, calorimetry, and muon detection over almost the entire solid angle [5]. The detectors are built with approximately cylindrical geometries, with a uniform magnetic field parallel to the beam inside the tracking volumes. Particularly important for heavy flavour physics are the high precision silicon microvertex detectors of the experiments - these have been added by all four collaborations since LEP started. Results using these precise vertex detectors have been published by ALEPH, DELPHI and OPAL, and analyses from L3, whose microvertex detector was installed last, are in progress.

The experimental method of measuring heavy quark partial widths and asymmetries proceeds by first isolating a pure sample of hadronic $\mathrm{Z}^{0}$ decays. Events containing heavy quarks are selected using various tagging techniques. A good understanding of these tagging methods is essential for precise electroweak measurements, and the techniques are therefore discussed in detail in this section. The partial width ratios $\Gamma_{Q} \bar{Q} / \Gamma_{\text {had }}$, where $Q$ is $b$ or c, can be derived straightforwardly from the number of tagged events and the total number of hadronic events, if the efficiencies are known. A more sophisticated technique, "double-tagging", allows the heavy quark tagging efficiency also to be derived from the data, and is described in section 4 . Forward-backward asymmetry measurements, on the other hand, also require that the axis of the $\mathrm{Z}^{0}$ decay to quark-antiquark pair be identified, and that the quark and antiquark be distinguished, in practice achieved using a charge measure. 
Hadronic $\mathrm{Z}^{0}$ decays can easily be identified at LEP. Backgrounds from $\mathrm{Z}^{0}$ decays to lepton pairs are rejected by cutting on the charged track or electromagnetic cluster multiplicities. Backgrounds, from cosmic rays, detector noise, beam-gas interactions, and two-photon processes, can be rejected by requiring a high visible energy in the event as expected from $\mathrm{Z}^{0}$ decay, that several tracks come from the interaction point, and that the momentum flow in the event is approximately balanced. The selection efficiencies for hadronic $\mathrm{Z}^{0}$ decays are typically between $95 \%$ and $99 \%$, and are known to the $0.5 \%$ level or better. Residual background comes mostly from $\mathrm{Z}^{0}$ decays to $\tau^{+} \tau^{-}$, and is typically at the level of $0.1-0.5 \%$. The quality of the hadronic event selection procedure is sufficient to ensure that consequent errors on the heavy quark electroweak measurements are negligible.

The four heavy quark tagging techniques employed are discussed in the following subsections. At present, lifetime and lepton tags are most powerful for $\mathrm{Z}^{0} \rightarrow \mathrm{b} \overline{\mathrm{b}}$ events, lepton and reconstructed particle tags for $\mathrm{Z}^{0} \rightarrow \mathrm{c} \overline{\mathrm{c}}$ events. The decaying quark charge can be estimated from the charges of leptons and reconstructed particles, when they are used as tags. For other tag types, such as vertex tags, a special technique is needed. This is provided by "hemisphere charge" estimators, as discussed in section 3.5.

The flight direction of a heavy quark needs to be estimated for two purposes. For the asymmetry measurement, the axis of the $\mathrm{Z}^{0} \rightarrow \mathrm{Q} \overline{\mathrm{Q}}$ decay must be estimated - this is customarily taken to be the event thrust axis. Most of the tagging techniques use the kinematic properties of heavy hadron decays, such as their large mass, to identify them. These approaches benefit greatly from a good estimate of the flight direction of the heavy hadron being tagged. This second type of direction estimate, of the decaying heavy hadron rather than the $Z^{0}$ decay axis, is usually obtained using a jet-finding algorithm, identifying jet directions with quark flight directions. The details of jet reconstruction vary from analysis to analysis, but a similar algorithm is used throughout.

The jet-finding algorithm takes as input a set of energy-momentum four-vectors, and clusters them into jets. The input four-vectors supplied approximate the individual final-state particles. ALEPH, DELPHI and OPAL use calorimeter energy deposits and reconstructed tracks to give the input four-vectors, removing energy matched with tracks to avoid double counting. L3 use calorimeter energy alone, as that performs just as well. These input "particles" are then clustered according to the jet-finding algorithm. Practically all analyses use an algorithm first proposed by the JADE collaboration [6]. This proceeds by considering all pairs of particles, $i$ and $j$, and calculating the invariant-mass-squared of each pair, $M_{i j}^{2}$, defined by:

$$
M_{i j}^{2}=2 E_{i} E_{j}\left(1-\cos \Theta_{i j}\right)
$$

where $E_{i}, E_{j}$ are the energies of the two particles, and $\Theta_{i j}$ is the angle between them. The pair with the lowest mass are merged into a single "pseudo-particle" with four-momentum equal to the sum of the four-momenta of the two constituents. The procedure is repeated until the masses of all particle and pseudo-particle pairs are greater than a cut-off threshold. The two most common variants of the scheme either apply a fixed mass-squared cut-off, $x_{\min }$, or a fixed cut-off, $y_{\text {cut }}$, on the mass-squared scaled by the visible energy in the event. These two schemes give similar performance, and the typical cut-offs used for heavy quark measurements are $x_{\min }=36-49 \mathrm{GeV}^{2}$, or alternatively $y_{\text {cut }}=0.01-0.02$. In $\mathrm{Z}^{0} \rightarrow \mathrm{b} \overline{\mathrm{b}}$ events, such jet-finding schemes give estimates of the $\mathrm{b}$ hadron direction with a typical resolution of about $70 \mathrm{mrad}$, improving to about $50 \mathrm{mrad}$ for jet energies above $10 \mathrm{GeV}$. 
In the discussions that follow in this section, it should be understood that the various heavy flavour tags can be applied to complete events, event hemispheres, or other restricted parts of events. For simplicity the tags are frequently discussed as applying to events - this should not be taken to imply any loss of generality. In practice, tags are often applied to event thrust hemispheres, defined as being separated by the plane through the interaction point perpendicular to the event thrust axis.

\subsection{Lifetime Tagging Techniques}

The weak decays of hadrons containing b or c quarks result in long lifetimes for these particles relative to strongly or electromagnetically decaying particles. The lifetimes are, in turn, much shorter than those of weakly decaying light hadrons. As indicated in table $1, \mathrm{~b}$ hadrons generally have longer lifetimes than $\mathrm{c}$ hadrons, giving correspondingly larger typical impact parameters. However, the average energies carried by first-rank $\mathrm{b}$ or $\mathrm{c}$ hadrons in $\mathrm{Z}^{0}$ decays do not differ much, being approximately $70 \%$ and $50 \%$ of the beam energy, respectively. Since c hadrons are lighter than $b$ hadrons, this results in rather similar typical decay lengths for both types of hadrons, of a couple of millimetres. Also important for separation of lifetime tags from $b$ and $c$ quarks are the masses of the decaying hadrons and their charged decay multiplicities. For $\mathrm{b}$ hadrons, the mean charged multiplicity has been measured, for example by OPAL to be $5.03 \pm 0.04 \pm 0.49[7]$, excluding $\mathrm{K}^{0}$ and $\Lambda$ decay products. The mean charged decay multiplicities have been measured by Mark-III for the $\mathrm{D}^{0}, \mathrm{D}^{+}$and $\mathrm{D}_{\mathrm{s}}^{+}$to be about $2.2[8]$. A substantial amount of separation can thus be achieved using information on the charged multiplicity in the heavy hadron decay.

\begin{tabular}{|c|c|c|c|c|}
\hline & $\begin{array}{c}\text { Lifetime } \\
\tau(\mathrm{ps})\end{array}$ & $\begin{array}{c}\text { Typical impact parameter } \\
c \tau(\mathrm{mm})\end{array}$ & $\begin{array}{c}\text { Typical boost } \\
\beta \gamma\end{array}$ & $\begin{array}{c}\text { Typical decay length } \\
\beta \gamma c \tau(\mathrm{mm})\end{array}$ \\
\hline \hline $\mathrm{B}^{+}$ & $1.54 \pm \mathbf{0 . 1 1}$ & $\mathbf{0 . 4 6}$ & 7 & 3.2 \\
$\mathrm{~B}^{0}$ & $1.50 \pm \mathbf{0 . 1 1}$ & $\mathbf{0 . 4 5}$ & 7 & 3.1 \\
$\mathrm{~B}_{\mathrm{s}}^{0}$ & $1.34_{-0.27}^{+0.32}$ & $\mathbf{0 . 4 0}$ & 7 & 2.8 \\
$\Lambda_{\mathbf{c}}$ & $1.07_{-0.16}^{+0.19}$ & $\mathbf{0 . 3 2}$ & 7 & 2.2 \\
\hline $\mathrm{D}^{+}$ & $1.057 \pm \mathbf{0 . 0 1 5}$ & $\mathbf{0 . 3 2}$ & 12 & 3.8 \\
$\mathrm{D}^{0}$ & $\mathbf{0 . 4 1 5 \pm 0 . 0 0 4}$ & $\mathbf{0 . 1 2}$ & 12 & 1.5 \\
$\mathrm{D}_{\mathrm{s}}^{+}$ & $\mathbf{0 . 4 6 7 \pm 0 . 0 1 7}$ & $\mathbf{0 . 1 4}$ & 12 & 1.7 \\
$\Lambda_{\mathbf{c}}$ & $\mathbf{0 . 2 0 0 _ { - 0 . 0 1 0 } ^ { + 0 . 0 1 1 }}$ & $\mathbf{0 . 1 4}$ & 12 & 0.7 \\
\hline
\end{tabular}

Table 1: Lifetimes, typical impact parameters, and typical decay lengths of $\mathrm{b}$ and $\mathrm{c}$ flavoured hadrons. Lifetimes are taken from the 1994 Review of Particle Properties [9]. Typical boosts correspond to those of $b$ or $c$ hadrons with the mean energy expected after fragmentation at LEP (hadron energy 0.7 or 0.5 times the beam energy, respectively).

Two rather different approaches are employed to make best use of lifetime information. In one, events are tagged if they have several tracks with significant impact parameters. In the other, an attempt is made to reconstruct explicitly the secondary vertex from the decaying heavy hadron, and to measure its decay length.

The high precision silicon microvertex detectors of the LEP experiments are able to resolve impact parameters and decay lengths on this scale. They have also been upgraded over time. 
In some analyses high precision microvertex information is available in three dimensions, in others only in the plane perpendicular to the beam axis. When this is the case, lifetime tag algorithms use impact parameters (or decay lengths) projected into this plane. This twodimensional problem is relatively straightforward, the full three-dimensional problem is rather more complex.

A common requirement in all the lifetime tag methods is for a precise estimate of the primary vertex position. This is obtained event by event by fitting reconstructed tracks to a common primary vertex point, discarding those which are inconsistent with coming from the primary. Tracks from heavy flavour decays will often be included in this primary vertex determination, leading to a mis-determined primary vertex. Such effects have to be considered in lifetime tag analyses, either explicitly, or by modifying the primary vertex position fit to make it insensitive to such tracks. The position and size of the LEP beam spot are usually included as constraints in the event-by-event primary vertex fit. The size and position of the beam spot are themselves determined by averaging over many events the primary vertex position found without the beamspot constraint. The spot position varies with time, and the size changes if the LEP machine optics are changed. The position is measured with a typical precision of $10 \mu \mathrm{m}$ in the plane transverse to the beam. The beam spot shape is highly elliptical in this plane, being around $5-10 \mu \mathrm{m}$ vertically and $100 \mu \mathrm{m}$ horizontally. The beam spot is typically $1 \mathrm{~cm}$ long.

In many of the analyses, it is necessary to use Monte Carlo simulated data to evaluate the performance of tagging algorithms. Much effort has been invested to ensure that the uncertainties so introduced do not dominate errors on results. For lifetime tags, use of simulated data is particularly important. Corrections are commonly applied to the simulated data to improve agreement with data, and it is customary to vary the size of these corrections to estimate detector resolution uncertainties.

\subsubsection{Impact Parameter Lifetime Tags}

The simplest lifetime tagging method is to require that there be some minimum number of tracks with impact parameter above a threshold. However, impact parameter resolution errors are sometimes comparable with the impact parameters being measured, so it is preferable to cut on the "impact parameter significance", $\mathcal{S}$, defined as the impact parameter divided by its measurement error.

In the two-dimensional case, the impact parameter, $b$, is defined as the distance of the track from the primary vertex at the point of closest approach of the track to the vertex, as shown on the left of figure 3 . The impact parameter is signed respectively positive, or negative, if the tangent to the track at the point of closest approach to the primary vertex crosses the axis in front of, or behind, the vertex. In the three-dimensional case, shown on the right in figure 3, the track curvature has been exaggerated to emphasise that tracks are approximately helical in three dimensions. In the ALEPH definition of the three-dimensional impact parameter [10], the tangent of the track helix is found at its point of closest approach, S, to the jet axis, and the impact parameter is defined as the perpendicular distance of this tangent from the primary vertex. The impact parameter is signed positive or negative according to whether this point of closest approach, $\mathrm{S}$, of the track helix to the axis is in front of, or behind, the primary vertex relative to the jet axis.

In both cases tracks with real lifetime information would usually be expected to have positive impact parameters. Signing errors can occur, however, because the axis chosen does not quite 


\section{2-d impact parameter}

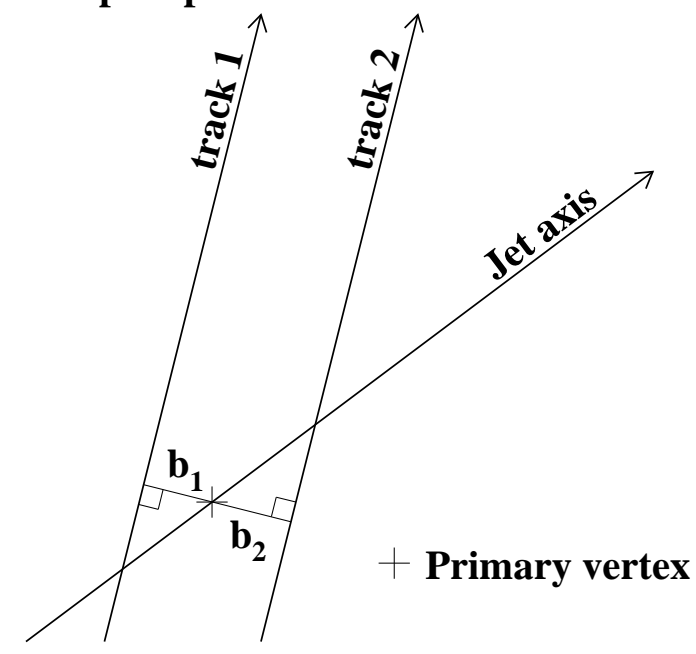

\section{3-d impact parameter}

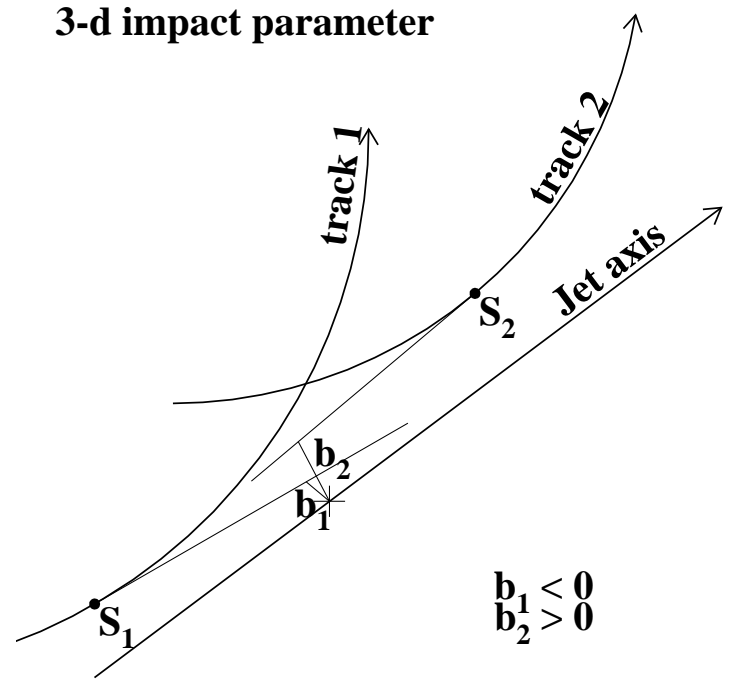

Figure 3: Impact parameter signing conventions. In the two-dimensional case the tracks are represented by their tangents at the point of closest approach to the vertex. In both cases shown, track 1 has a negative signed impact parameter, $b_{1}<0$, and track 2 a positive value, $b_{2}>0$.

correspond to the true decaying particle direction. Such signing errors have the undesirable feature of introducing an exponential lifetime component into the negative impact parameter significance distribution.

The typical impact parameter resolution obtained for tracks with microvertex detector information is $15-25 \mu \mathrm{m}$ for $45 \mathrm{GeV}$ tracks. At lower momentum the resolution decreases because of multiple scattering in material around the beam-pipe, typically to around $70 \mu \mathrm{m}$ at $2 \mathrm{GeV}$.

The number of tracks with signed impact parameter significance, $\mathcal{S}$, greater than some given threshold is termed the "forward multiplicity". Forward multiplicity was first used for b tagging in $\mathrm{Z}^{0}$ decays by Mark-II [11], and has also been used by OPAL to measure $\Gamma_{\mathrm{bb}} / \Gamma_{\text {had }}[12]$.

A more sophisticated way of using impact parameter information has been devised by ALEPH [10]. This proceeds by estimating the probability, $\mathcal{P}_{T}$, that each track with a positive signed impact parameter significance, $\mathcal{S}>0$, is actually a track from the primary vertex. The measure $\mathcal{P}_{T}$ is defined by

$$
\mathcal{P}_{T}=\int_{\mathcal{S}}^{\infty} \mathcal{R}(x) \mathrm{d} x
$$

where $\mathcal{R}$ is the probability density function for $\mathcal{S}$, resulting entirely from resolution effects. By construction $\mathcal{P}_{T}$ should have a flat probability distribution between 0 and 1 for tracks genuinely produced at the primary vertex. The $\mathcal{P}_{T}$ values of all selected tracks in the event, event hemisphere, or jet (as appropriate for the analysis considered) are then combined to form a joint probability, $\mathcal{P}_{N}$, for that group of tracks. The quantity $\mathcal{P}_{N}$ is defined as

$$
\mathcal{P}_{N} \equiv \Pi \sum_{j=0}^{N-1} \frac{(-\ln \Pi)^{j}}{j !}
$$


where

$$
\Pi \equiv \prod_{i=1}^{N}\left(\mathcal{P}_{T}\right)_{i}
$$

The quantity $\Pi$ is just the product over the $N$ tracks of the probabilities that each originates from the primary vertex. This quantity itself contains the information required about the nolifetime likelihood of the particular set of impact parameter significances observed, but it is not a very useful variable as its distribution depends strongly on the number of tracks $N$ contributing. To avoid such problems, $\mathcal{P}_{N}$ is constructed as the probability of finding the particular set of impact parameter significances obtained, or any equally or less likely configuration, for this particular value of $N$. In this way a variable is constructed which, in the case of no lifetime, is flat between 0 and 1 for each value of $N$ separately. For events with lifetime information $\mathcal{P}_{N}$ is close to zero. Of course, there is lifetime information even in light quark events from $\mathrm{K}^{0}$ and $\Lambda$ decays. This is reduced by rejecting identified photon conversion, $\mathrm{K}^{0}$ and $\Lambda$ decay candidates.

For ALEPH, this technique is found to provide a very powerful tagging algorithm. The power of the technique lies in the low probability that large impact parameter significances can come from tracks actually originating from the primary vertex - so it is critical that the tails of the resolution function for the impact parameter significance are kept small, and are well understood. Since the product of probabilities is taken over several tracks, and the value of $\mathcal{P}_{T}$ for a given track varies directly with the size of these tails, small reductions in the size of the tails can give significant improvements in the tagging performance. Careful study is therefore necessary to reduce as far as possible poorly-measured or badly reconstructed tracks. The size of the resolution tails can be measured from the data using negative signed impact parameter tracks, allowing this source of systematic uncertainty to be reasonably well controlled. The effects of impact parameter signing errors mean that care has to be taken to ensure the real lifetime contamination in this backward distribution is not too high. The performance of this type of algorithm varies significantly from one LEP detector to another. DELPHI have also adopted this impact parameter tagging algorithm $[13,14]$.

\subsubsection{Reconstructed Secondary Vertex Tags}

OPAL have used a different type of lifetime tagging algorithm, based on secondary vertex reconstruction [15]. This algorithm is relatively insensitive to resolution tails in impact parameter distributions, as several tracks are needed to form the secondary vertex, and they must all be reasonably consistent with coming from that common secondary vertex. Published analyses have so far been restricted to vertex reconstruction in the plane perpendicular to the beam.

The algorithm used by OPAL is a "tear-down" vertex finder, operating on tracks which have been clustered into jets. Track quality cuts are applied to reduce contamination from poorly measured tracks, and tracks from $\mathrm{K}^{0}$ and $\Lambda$ decays. Selected tracks in the jet are fitted to a common vertex point, and the $\chi^{2}$ of the vertex fit is evaluated. The change in this $\chi^{2}, \Delta \chi^{2}$, is evaluated when each track in the vertex is dropped in turn. If any track contributes $\Delta \chi^{2}>4$ the track giving the largest $\Delta \chi^{2}$ is dropped, and the fit repeated. The process is iterated until no tracks contribute $\Delta \chi^{2}>4$, or until fewer than four charged tracks remain, in which case no vertex is found.

The decay length, $L$, is defined as the distance from the reconstructed secondary vertex position to the primary vertex, constrained by the direction of the jet (or by the direction of 


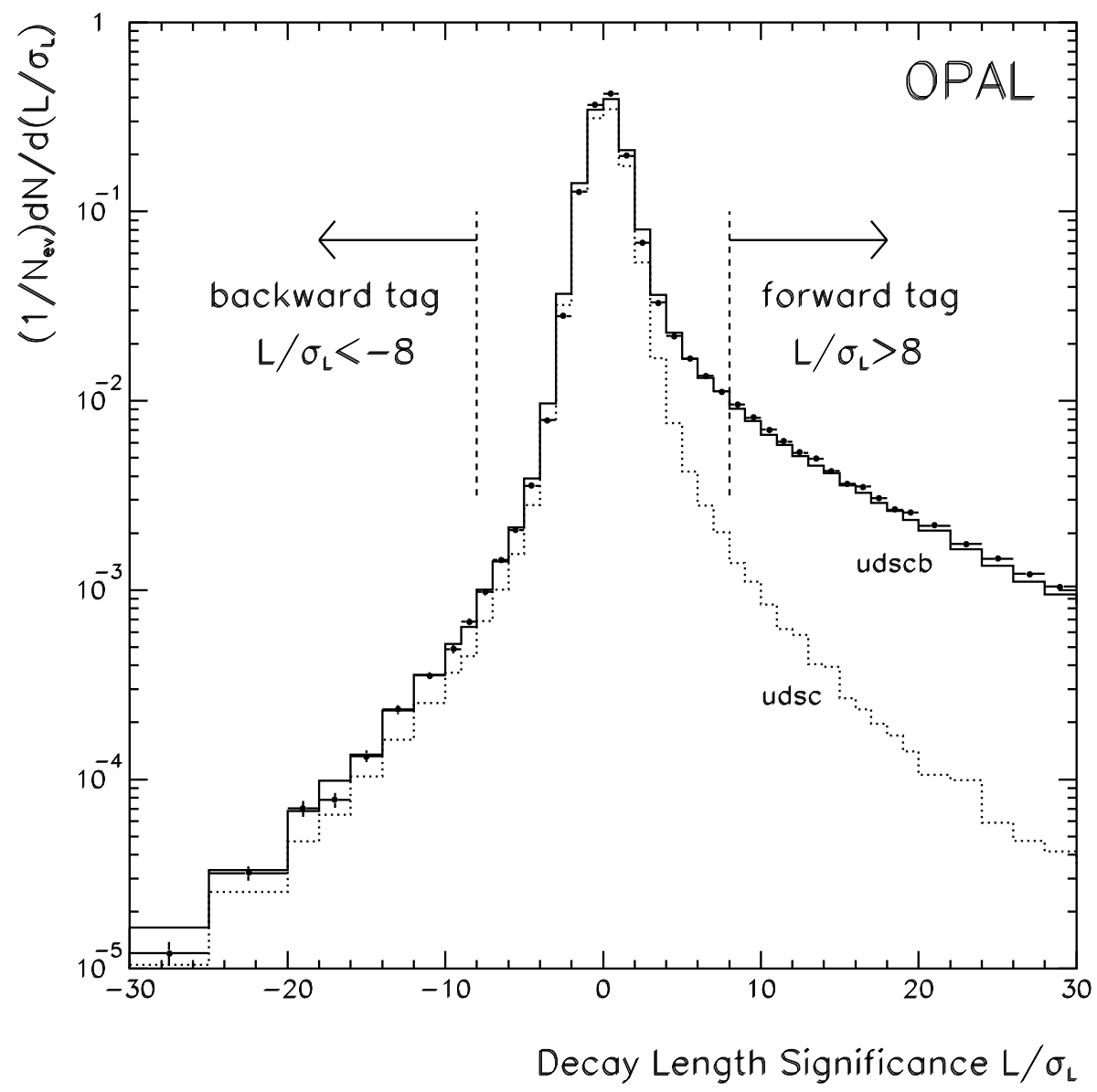

Figure 4: Decay length significance, $L / \sigma_{L}$, distribution for OPAL data and Monte Carlo simulation.

summed momentum vectors of the particles associated with the secondary vertex). The decay length is signed positive if the secondary vertex is displaced in front of the primary vertex relative to the constraining direction, negative otherwise. The error, $\sigma_{L}$, on the decay length includes the errors from both the primary and secondary vertex reconstruction uncertainties. To reduce the effect of poorly measured vertices, the "decay length significance", $L / \sigma_{L}$, is used as the lifetime tag discriminant, typically requiring $L / \sigma_{L}>8$ for a b tag.

This secondary-vertex reconstruction algorithm has the desirable property that it gives a symmetric decay length significance distribution for events with no lifetime information. A second useful feature is that small shifts in the direction constraint used to sign the decay length cannot give rise to decay length signing errors - rather they just degrade the decay length significance resolution somewhat.

The distribution of $L / \sigma_{L}$ obtained by OPAL is shown in figure 4 . The lifetime information in the forward direction coming from b decays is clearly visible. The agreement between simulation and data in the backward direction, dominated by resolution effects, is seen to be reasonable for decay length significances $L / \sigma_{L}<-5$. A simple way of reducing the effects of resolution uncertainties coming from background with no lifetime component is to subtract the backward tags observed in the data from the forward tags at the same value of $\left|L / \sigma_{L}\right|$. This works well, but is only appropriate in a subset of analyses, due to its statistical nature. 


\subsubsection{Lifetime Tag Performance}

\begin{tabular}{|l|c|c|}
\hline & b efficiency $\varepsilon^{\mathrm{b}}(\%)$ & b purity $(\%)$ \\
\hline \hline ALEPH [10] & 26 & 96 \\
DELPHI [13] & 24 & 89 \\
OPAL [15] & 19 & 95 \\
\hline
\end{tabular}

Table 2: Efficiencies and purities of $b$ quark hemisphere tags using lifetime information. The efficiencies quoted are for a hemisphere of the event - the efficiency for tagging either hemisphere of an event is higher, being approximately $2 \varepsilon^{\mathrm{b}}-\left(\varepsilon^{\mathrm{b}}\right)^{2}$.

The performance of the tagging algorithms described is shown in table 2. The $\mathrm{b}$ tagging efficiencies, $\varepsilon^{\mathrm{b}}$, shown in the table are all derived from the data themselves, using the doubletagging method described in section 4 below. The double-tag technique essentially removes uncertainties on $\varepsilon^{\mathrm{b}}$ from $\mathrm{b}$ decay modelling, substantially reducing systematic errors. Because $\mathrm{c}$ hadrons have similar lifetimes to $\mathrm{b}$ hadrons (table 1 ), the impurities in $\mathrm{b}$ tagged samples arise predominantly, typically about $70 \%$, from $\mathrm{Z}^{0} \rightarrow \mathrm{c} \overline{\mathrm{c}}$ events. The systematic errors on the $\mathrm{b}$ efficiency and purity are dominated by uncertainties on the modelling of $\mathrm{c}$ hadron decays. Systematic errors from uncertainties on the resolution modelling are quite effectively reduced by the techniques described above, but are still significant.

In summary, table 2 shows that lifetime tags are very powerful b hadron indicators. Purities of $95 \%$ are attainable with hemisphere efficiencies of $20-25 \%$ - this is substantially better than any other tagging technique available.

\subsection{Lepton Tagging}

The semileptonic decays of $\mathrm{b}$ and $\mathrm{c}$ hadrons to electrons and muons provide a long-established technique for heavy flavour tagging. The large mass and hard fragmentation of heavy hadrons at LEP result in high momentum leptons from their decays. In the case of b decays, the leptons typically also have significant momentum component relative to the decaying hadron direction, approximated in practice by the direction of the jet containing the lepton. Events containing $\mathrm{b}$ hadrons can therefore be tagged by requiring that there be an identified electron or muon with high momentum, $p$, and high transverse momentum, $p_{t}$, relative to the containing jet. At LEP, most leptons with $p>2 \mathrm{GeV}$ come from decays of $\mathrm{b}$ or $\mathrm{c}$ hadrons, but these give different lepton spectra, allowing a statistical separation over the full $p_{t}$ range. This type of analysis is quite powerful, as systematic uncertainties can be reduced by fitting for the different lepton source components, and is discussed at more length below. Semileptonic decays to $\tau$ leptons are not usable as lepton tags because of the difficulty in identifying their decay products in the hadronic event environment, although inclusive $\mathrm{b}$ decays to $\tau$ have now been measured from the missing momentum spectrum [16]. In the following, "lepton", $\ell$, therefore refers to electrons and muons only.

Several channels contribute to the inclusive lepton yield:

- Semileptonic b decays, $\mathrm{b} \rightarrow \ell^{1}$

\footnotetext{
${ }^{1}$ In this paper discussions of specific particle decays and reconstructed particles should be taken also to refer
} 
- "Cascade" b decays, $\mathrm{b} \rightarrow \mathrm{c} \rightarrow \ell$ and $\mathrm{b} \rightarrow \overline{\mathrm{c}} \rightarrow \ell$

- Decays of $\mathrm{b}$ hadrons to leptonically decaying $\mathrm{J} / \psi$ mesons, $\mathrm{b} \rightarrow \mathrm{J} / \psi \rightarrow \ell$

- Decays of $\mathrm{b}$ hadrons to a leptonically decaying $\tau, \mathrm{b} \rightarrow \tau \rightarrow \ell$

- Semileptonic c decays, $\mathrm{c} \rightarrow \ell$

- "Instrumental" backgrounds, from photon conversions to $\mathrm{e}^{+} \mathrm{e}^{-}$, decays in flight of $\pi$ and $\mathrm{K}$ to muons, hadrons faking lepton signatures, and so on.

The first five sources are together known as "prompt" lepton sources. Semileptonic b and c decays together with cascade decays produce the large majority of prompt leptons in the sample. Instrumental background sources are mostly concentrated at low $p$ and $p_{t}$.

(a) $\mathbf{b} \rightarrow \mathbf{l}$

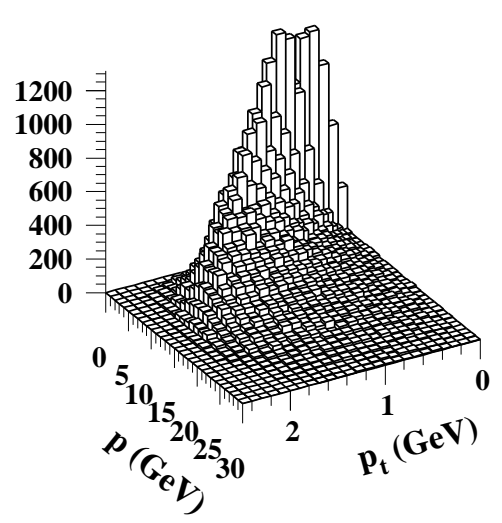

(b) $\mathbf{b} \rightarrow \mathbf{c} \rightarrow \mathbf{l}$

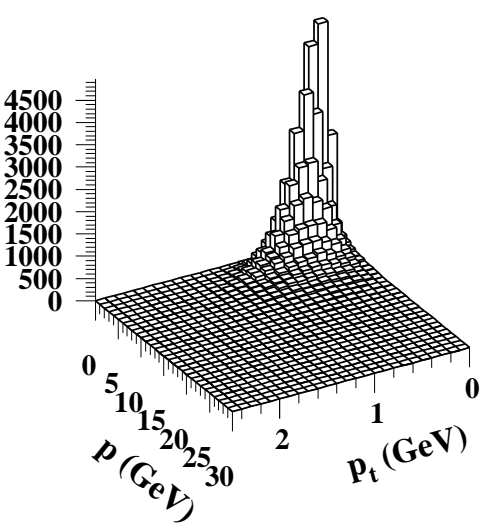

(c) $\mathbf{c} \rightarrow \mathbf{l}$

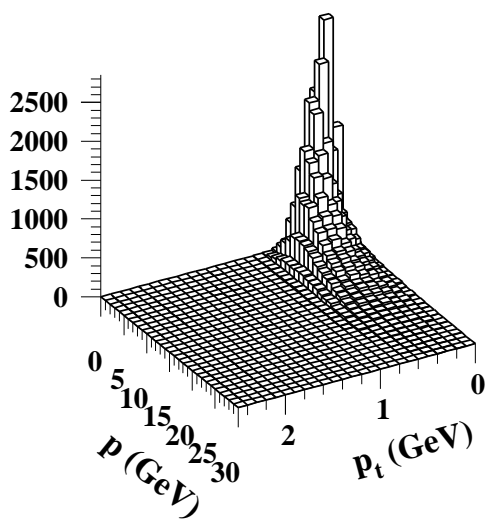

Figure 5: Distributions of $p$ vs. $p_{t}$, predicted by the JETSET Monte Carlo [4], for leptons from (a) semileptonic b decays; (b) cascade decays; and (c) semileptonic $\mathrm{c}$ decays in $\mathrm{Z}^{0}$ to co decay events. The momenta are defined in the experimental frame, and $p_{t}$ is measured relative to the decaying $\mathrm{b}$ hadron direction (for (a) and (b)) or relative to the decaying $\mathrm{c}$ hadron direction (for (c)). The vertical scale is arbitrary.

The possibility of flavour separation using lepton $p$ and $p_{t}$ distributions is illustrated in figure 5. Leptons directly from semileptonic $\mathrm{b}$ decays have harder $p$ and $p_{t}$ distributions than those from cascade and semileptonic $\mathrm{c}$ decays. In turn, leptons from semileptonic $\mathrm{c}$ decays in $\mathrm{Z}^{0} \rightarrow \mathrm{c} \overline{\mathrm{c}}$ events have a harder $p$ spectrum than those from cascade decays. The reconstructed $p$ and $p_{t}$ distributions for identified muons in the L3 detector are shown in figure 6 . Background leptons are only a small component for $p_{t}>1 \mathrm{GeV}$. For this L3 analysis, the $p_{t}$ of the lepton is calculated relative to its containing jet, excluding the lepton momentum when calculating the jet direction.

Fitting the inclusive lepton spectrum can provide some information about electroweak parameters, but much can be gained if events with two identified leptons ("dileptons") are also to the corresponding charge-conjugated process or particles. 

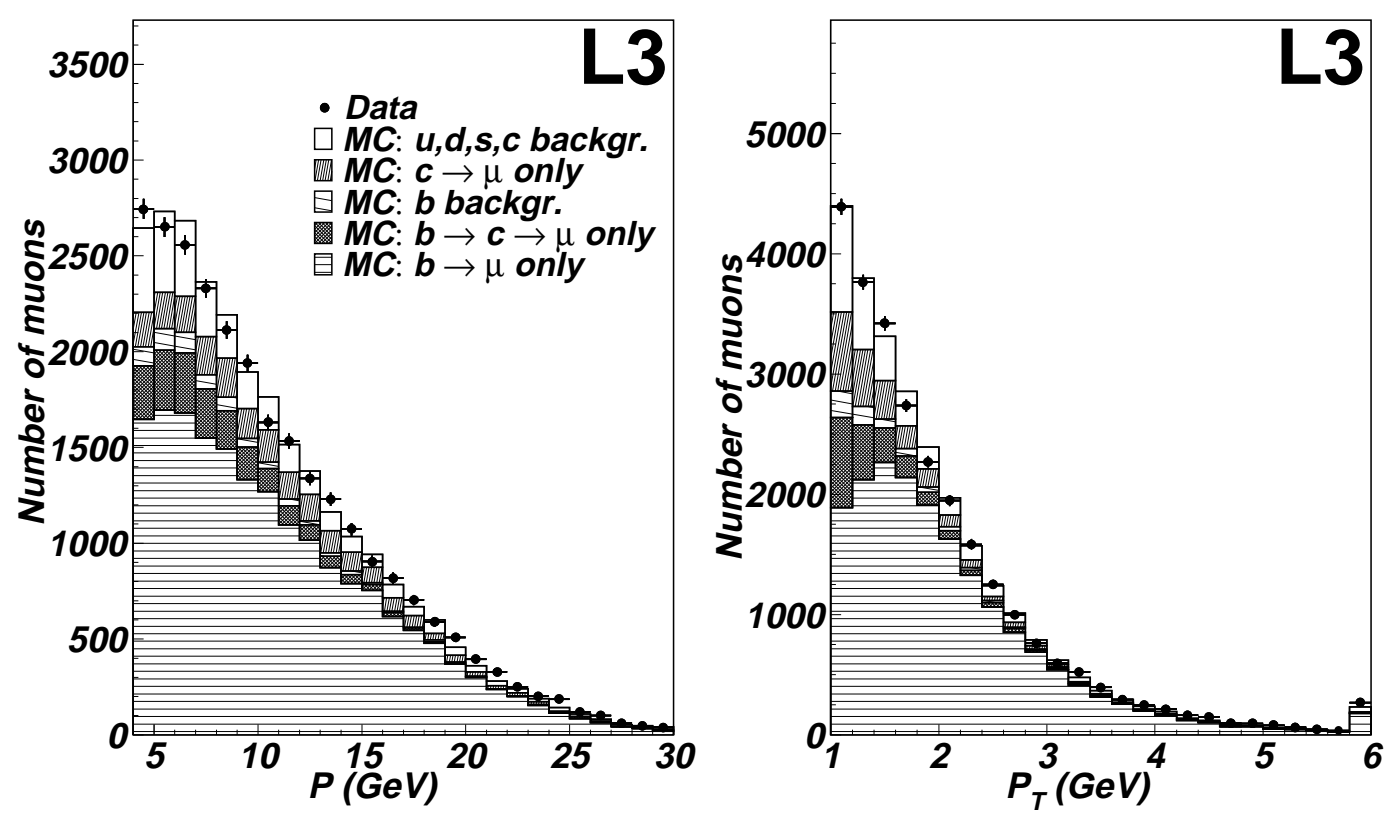

Figure 6: Distributions of $p$ and $p_{t}$ for muons reconstructed in the L3 detector [17], compared to Monte Carlo (MC) predictions. Overflow entries are shown in the rightmost bins. Predicted background lepton candidates are shown separately for $b \bar{b}$ and non- $b \bar{b}$ events.

used. By fitting both the lepton and dilepton spectra together, more quantities can be measured simultaneously. If instrumental backgrounds are neglected (or first subtracted from the data), the inclusive differential cross-section in $p$ and $p_{t}$ for prompt leptons has the form:

$$
\frac{\mathrm{d}^{2} \sigma_{\ell}}{\mathrm{d} p \mathrm{~d} p_{t}} \propto \sum_{i} B(i) \varepsilon_{i}\left(p, p_{t}\right) \frac{\Gamma_{\mathrm{b} \overline{\mathrm{b}}}}{\Gamma_{\mathrm{had}}} f_{i}\left(p, p_{t}\right)+B(\mathrm{c} \rightarrow \ell) \varepsilon_{\mathbf{c} \rightarrow \ell}\left(p, p_{t}\right) \frac{\Gamma_{\mathbf{c} \overline{\mathbf{c}}}}{\Gamma_{\mathrm{had}}} f_{\mathrm{c} \rightarrow \ell}\left(p, p_{t}\right)
$$

where the sum over $i$ runs over the first four lepton source channels listed above, $B(i)$ represents the decay branching ratio for source $i, \varepsilon_{i}\left(p, p_{t}\right)$ refers to the efficiency of detecting a lepton from source $i$, and $f_{i}\left(p, p_{t}\right)$ represents the $p, p_{t}$ distribution of the leptons from source $i$. For dileptons, if it is assumed that the two leptons originate in different heavy quark decays, and the efficiency correlation between the two leptons is neglected, the differential cross-section takes the form:

$$
\begin{aligned}
\frac{\mathrm{d}^{4} \sigma_{\ell}}{\mathrm{d} p_{1} \mathrm{~d} p_{t, 1} \mathrm{~d} p_{2} \mathrm{~d} p_{t, 2}} \propto & \sum_{i, j} B(i) B(j) \varepsilon_{i}\left(p_{1}, p_{t, 1}\right) \varepsilon_{j}\left(p_{2}, p_{t, 2}\right) \frac{\Gamma_{\mathrm{b} \overline{\mathrm{b}}}}{\Gamma_{\mathrm{had}}} f_{i}\left(p_{1}, p_{t, 1}\right) f_{j}\left(p_{2}, p_{t, 2}\right)+ \\
& B(\mathrm{c} \rightarrow \ell)^{2} \varepsilon_{\mathbf{c} \rightarrow \ell}\left(p_{1}, p_{t, 1}\right) \varepsilon_{\mathbf{c} \rightarrow \ell}\left(p_{2}, p_{t, 2}\right) \frac{\Gamma_{\mathbf{c} \overline{\mathbf{c}}}}{\Gamma_{\mathrm{had}}} f_{\mathbf{c} \rightarrow \ell}\left(p_{1}, p_{t, 1}\right) f_{\mathbf{c} \rightarrow \ell}\left(p_{2}, p_{t, 2}\right)
\end{aligned}
$$

where now the $p_{1 / 2}$ and $p_{t, 1 / 2}$ of both leptons (1/2) appear. The identification efficiencies $\varepsilon$ are known from simulation, and are checked with control samples of data. The momentum distributions $f$ are also known from a combination of lower energy experimental measurements and Monte Carlo simulation. Using the inclusive lepton distribution (equation (13)) alone, it is not possible to measure both the branching ratios and $\mathrm{Z}^{0}$ decay widths - a measurement of $\Gamma_{\mathrm{b}} / \Gamma_{\text {had }}$ requires that the branching ratio $B(\mathrm{~b} \rightarrow \ell)$ is known. This branching ratio is not known very precisely from lower energy data, so the systematic error on $\Gamma_{\mathrm{b}} / \Gamma_{\text {had }}$ from such an approach would be too big to be interesting. If, however, the single lepton and dilepton spectra 
are fitted together, the most important branching ratios can be determined from the data while simultaneously determining $\Gamma_{\mathrm{b}} / \Gamma_{\text {had }}$ and $\Gamma_{\mathbf{c} \overline{\mathrm{c}}} / \Gamma_{\text {had }}$.

Such fits to the single lepton and dilepton spectra are performed by all four LEP collaborations [14,17-19]. By extending the fit to include the $\cos \theta$ variation of the cross-section, the four electroweak heavy quark flavour parameters $\Gamma_{\mathrm{b}} / \Gamma_{\text {had }}, \Gamma_{\mathbf{c} \overline{\mathbf{c}}} / \Gamma_{\text {had }}, A_{\mathrm{FB}}^{\mathrm{b}}$ and $A_{\mathrm{FB}}^{\mathrm{c}}$ can, in principle, all be measured simultaneously. In practice only ALEPH have published such a measurement $[19]$ to date. The average $\mathrm{b}$ mixing parameter $\chi$, and the $\mathrm{b}$ fragmentation, may also be determined in these fits. The choice of exactly which parameters to measure, and which to take from external sources, such as lower energy measurements, is a balance which depends on the statistics used in the fit and the details of the approach used. The approaches adopted by the four collaborations differ - ALEPH prefer a combined fit of all parameters, whereas the other experiments have separate fits for smaller sets of parameters.

The major sources of systematic errors in lepton analyses come from semileptonic decay properties. The large uncertainties which would arise from knowledge of the $b$ semileptonic branching ratio are removed by fitting for it, but the momentum spectrum of the lepton from the decay must still be assumed. Phenomenological models [20,21] are used to predict the spectrum. The models have been tuned to reproduce the momentum spectrum of leptons from decays of slow B mesons, produced in $\Upsilon(4 \mathrm{~S})$ decays observed by the CLEO detector [22]. Additional systematic errors accrue because of the admixture of unmeasured semileptonic $B_{s}^{0}$ and $\Lambda_{c}$ decays. Errors arise in a similar way for semileptonic c decays, where the most useful lower energy measurements come from DELCO and MARK-III [23, 24].

Experimental systematic errors arise from knowledge of the lepton identification efficiencies, and the contamination by instrumental backgrounds. Lepton identification efficiencies are generally very well known at LEP, typically at the level of $\pm 2 \%$, and do not give rise to significant uncertainties. Instrumental backgrounds, on the other hand, are pervasive and relatively difficult to estimate. Conversion background can be measured, and partially excluded, by reconstruction of the other electron track from the converting photon. Control samples of hadron tracks can be used to measure the modelling of hadronic backgrounds, but relative uncertainties remain at the level of $\pm 10 \%$.

The overall performance of lepton tags is limited by the low semileptonic branching ratios, of approximately $10 \%$ for both $\mathrm{b}$ and $\mathrm{c}$ semileptonic decays. Clean identification means that only leptons with $p>2$ or $3 \mathrm{GeV}$ can be used - a cut at $3 \mathrm{GeV}$ removes approximately $25 \%$ and $40 \%$ of leptons from primary $\mathrm{b}$ and $\mathrm{c}$ semileptonic decay, respectively. In this momentum range lepton identification efficiencies lie typically in the range $70-80 \%$. A close to $90 \%$ pure $\mathrm{b}$ tag requires typically an additional $p_{t}$ cut of about $1.2 \mathrm{GeV}$, which imposes a further efficiency loss of around $50 \%$, giving overall hemisphere tagging efficiencies of around $6 \%$, for a combined electron and muon tag.

\subsection{Flavour Separation using Event Shapes}

The high mass and hard fragmentation of the $b$ quark is exploited by another separation technique. Very little energy is lost by gluon radiation in the hadronisation process of b quarks, so that the $b$ hadron and, subsequently, its decay products, carry a large fraction of the primary quark energy. In contrast, in light quark events most particles are produced in gluon frag-

mentation with a rather soft momentum spectrum. Similarly, the b decay products can have 
a relatively large transverse momentum with respect to the $b$ quark direction, since the large mass of the $b$ hadron increases the available transverse phase-space significantly. This results in broader jets in $\mathrm{b} \overline{\mathrm{b}}$ events compared to light quark events.

Even without completely reconstructing the b decay products, quantities can be devised which carry some of this information. The hard $b$ fragmentation means that most information is carried by a small number of tracks with high momentum and high transverse momentum, measured relative to the jet direction. Many of the separating variables constructed are therefore restricted to a few of the most energetic tracks in a jet. ALEPH $[25,26]$ and OPAL [27] use very similar variables, constructed mostly from the momenta of tracks. The L3 collaboration [28] perform an equivalent analysis based entirely on calorimeter information. Typical quantities used are:

- $\mathcal{B}_{\text {jet }}$ : The boosted sphericity of the jet. The tracks of a jet are boosted into a new frame, such that the sphericity of $b$ decay products is isotropically distributed. Non-b jets, boosted into an equivalent frame, show different distributions.

- Quantities constructed from the momenta (or energies) of all tracks and of the most energetic ones, measured both transverse and longitudinally to the jet direction.

- $D_{123}, M_{123}$, the directed sphericity and invariant mass of the three most energetic particles in the jet;

- L3 includes the energy difference between the highest and the fourth highest energetic cluster, $E_{\text {gap }}$.

- ALEPH has constructed two additional variables which use all tracks in a hemisphere, rather than in a jet. They are constructed from the product of momenta of different tracks, and from the invariant masses assuming the pion mass for each track, calculated in the rest frame of all particles in the hemisphere.

Distributions of some of these quantities are shown in figure 7. The measured distributions are compared to the predicted behaviour of $b \bar{b}$ and $c \bar{c}$ events, with appropriate normalisation. None of these quantities by itself provides a very good separation between $b \bar{b}$ and non- $b \bar{b}$ events. In addition the variables are highly correlated, further complicating the analysis. To exploit fully the information available, and still take the correlations properly into account, an artificial neural net [29] is used to combine the variables into a single discriminating quantity. This is constructed such that, given perfect separation between flavours, it would be one for $b \bar{b}$ events, zero for c $\bar{c}$ events. The output distribution of such a network is shown in figure 8(a), and compared with simulated $b \bar{b}, c \bar{c}$ and light flavour events. The performance possible with such a net is illustrated in figure $8(\mathrm{~b})$, showing the efficiency versus purity curve obtained by L3 with a net using 11 variables per event.

DELPHI [30] have extended this method to attempt to separate b, c and uds events into three categories. To improve the separation power of the network, lifetime information is included in the form of impact parameters of leading tracks in the jet. The sensitivity to $c \bar{c}$ events is improved by using information typical for the decay of a charged $\mathrm{D}^{*+}$ meson into a $\mathrm{D}^{0}$ and a pion, of which the transverse momentum relative to the jet direction is expected to be very small. 

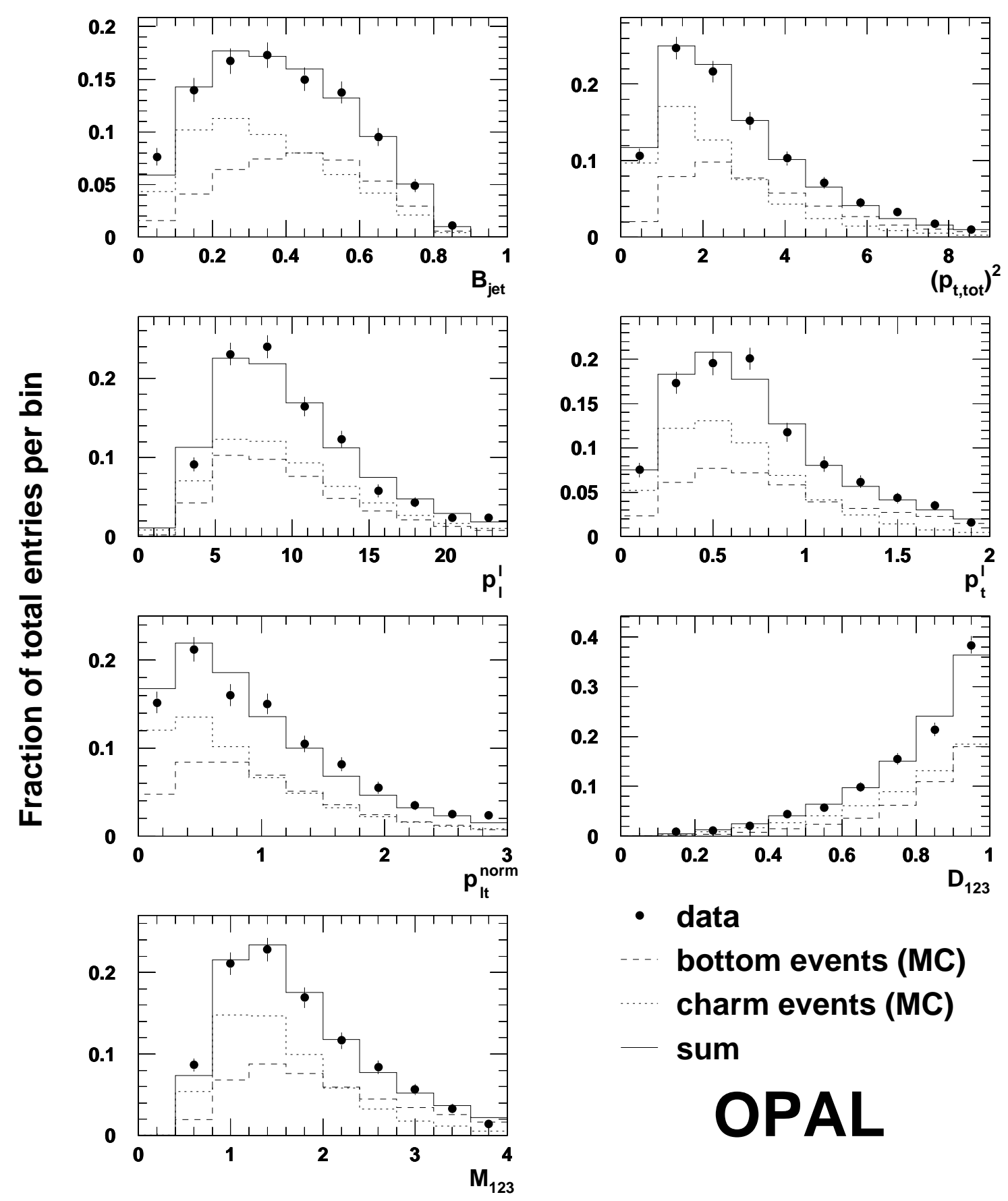

- data bottom events (MC) charm events (MC) sum OPAL

Figure 7: Distributions of the seven jet shape variables used by OPAL, for events with an exclusively reconstructed $\mathrm{D}^{*+}$ in the other hemisphere. Shown are data (points with error bars), Monte Carlo $\mathrm{b} \overline{\mathrm{b}}$ events (dashed line) and c $\bar{c}$ events (dotted line). Light flavour background has already been subtracted. 

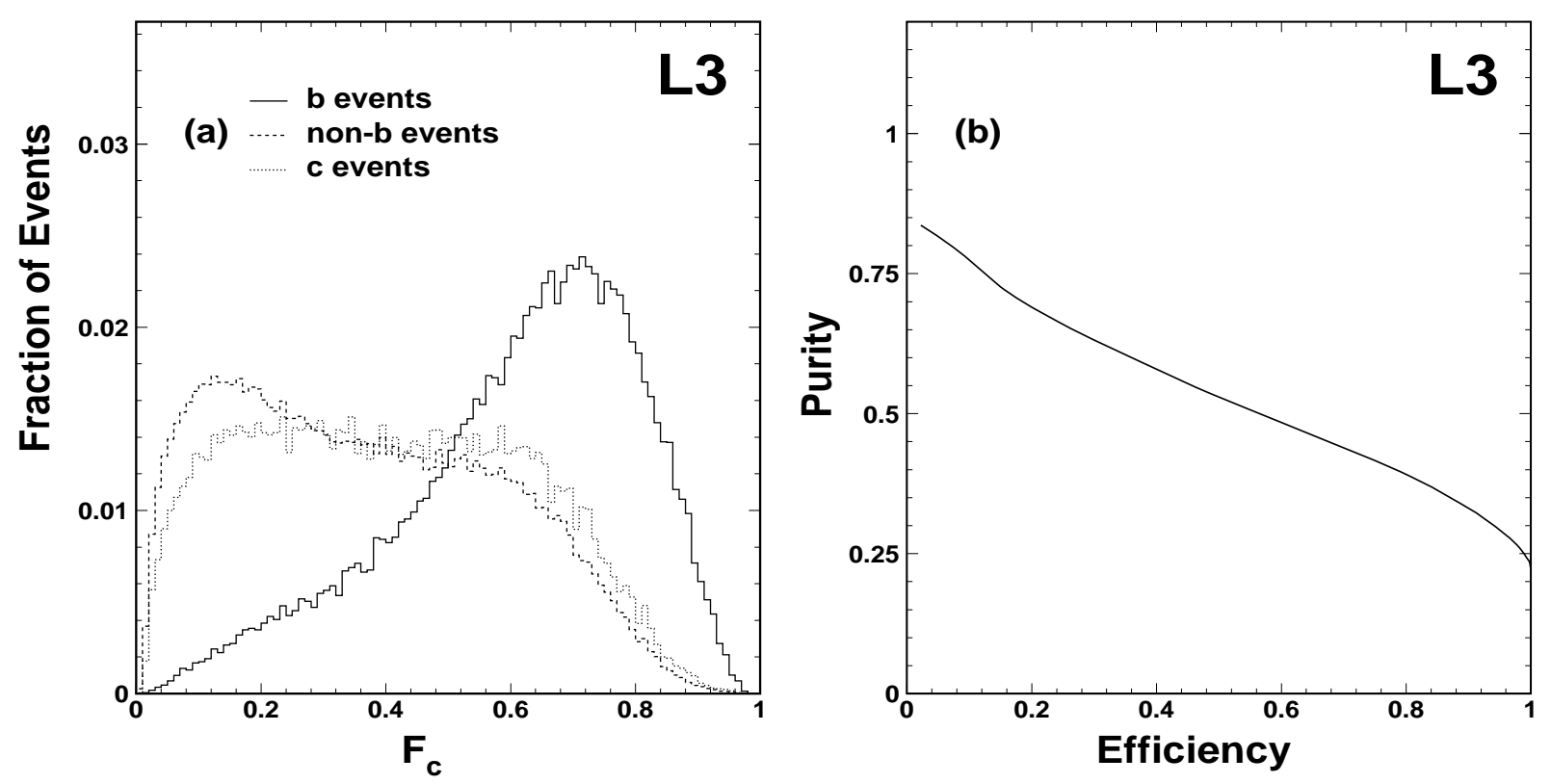

Figure 8: (a) Output distribution of the neural net used by the L3 collaboration [28] to identify $b \bar{b}$ events. The solid line is the distribution of $\mathrm{b} \overline{\mathrm{b}}$ events predicted by Monte Carlo, the dashed line that of non-b events, and the dotted line the that of c $\bar{c}$ events. (b) Efficiency vs. purity curve for the same net.

The network identification probabilities need to be well known for this approach to be useful. To minimise dependence on Monte Carlo modelling, attempts are made to measure these probabilities from the data, or to verify the probabilities found in the Monte Carlo. The response to $\mathrm{b} \overline{\mathrm{b}}$ events can be tested with lepton tagged samples. Large lepton samples exist with high purities and small systematic uncertainties, and are used to check the net response (ALEPH, DELPHI, L3) or to train the network (ALEPH, OPAL). ALEPH have developed a method of determining the network identification probabilities from the simultaneous detection of leptons in the opposite hemisphere [26].

Estimation of the network tagging rates is considerably more difficult for other flavours, since no unbiased pure data samples of charm or light flavour events exist. Therefore Monte Carlo has to be used to calculate the network response. The dependence of the results on the simulation is potentially one of the more important sources of systematic errors.

It is seen from figure $8(\mathrm{~b})$ that it is not possible to prepare very pure samples of a single flavour with an event shape tag. For $\mathrm{b} \overline{\mathrm{b}}$ events, purities of around $70 \%$ can be obtained with an event efficiency of about $20 \%$. The strength of the method lies in the relatively high efficiency obtainable with this type of tag, and, potentially, in its extension to include flavours other than bottom. 


\subsection{Reconstructed Particle Tags}

Reconstructed particles are a powerful tool for tagging the flavour of an event. In principle such tags could be designed for all five flavours. The idea is to use mesons or baryons ${ }^{2}$ which contain the flavour investigated, or which are the immediate decay products of particles containing this flavour. It is comparatively easy to tag $c \bar{c}$ and $b \bar{b}$ events because of the low production rate of these quarks in the fragmentation process. In many cases in such events the mesons sought contain the primary quark flavour, and so tag it. Although similar tags could be devised for the lighter flavours, interpretation of the measured particle rates in terms of the primary event flavours is difficult because a substantial fraction of such mesons would be produced in the hadronisation process.

Signals from fully reconstructed decays are usually fairly clean, so providing reasonably pure tags. However, in most cases analyses are limited by the small branching ratios into the identifiable channels, typically of the order of one percent. This, together with finite reconstruction efficiencies, restricts the precision obtainable in analyses based on fully reconstructed particles.

At LEP reconstructed particle tags are most important for charm tagging, although, since b hadrons nearly always decay to $\mathrm{c}$ hadrons, reconstructed c-flavoured particles can also be used to tag $\mathrm{b} \overline{\mathrm{b}}$ events. However, lifetime and lepton tags are much more powerful $\mathrm{b}$ tagging tools than reconstructed particles. When used as a charm tag, reconstructed particles from $b \bar{b}$ events are a background source which must be understood. Separation of the $b \bar{b}$ and $c \bar{c}$ components in the samples is discussed in more detail below.

The main decay channels used for electroweak analyses at LEP are listed in table 3. The branching ratios of each decay, and the expected number of mesons per hadronic $\mathrm{Z}^{0}$ decay in each channel, are also shown. Analyses using $\mathrm{D}^{*+}$ mesons are most advanced of those exploiting exclusive decay reconstruction, and so $\mathrm{D}^{*+}$ reconstruction techniques are emphasised in the following discussion.

\begin{tabular}{|c|c|c|}
\hline decay mode & branching ratio $(\%)$ & decays per $\mathrm{Z}^{0}$ \\
\hline $\mathrm{D}^{*++} \rightarrow \mathrm{D}^{0} \pi^{+}$ & $68.1 \pm 1.3$ & 0.1496 \\
\hline$\hookrightarrow \mathrm{K}^{-} \pi^{+}$ & $2.73 \pm 0.11$ & 0.0032 \\
\hline$\hookrightarrow \mathrm{K}^{-} \pi^{+} \pi^{0}$ & $9.40 \pm 0.70$ & 0.0111 \\
\hline$\hookrightarrow \mathrm{K}^{-} \pi^{+} \pi^{-} \pi^{+}$ & $5.52 \pm 0.36$ & 0.0065 \\
\hline $\mathrm{D}^{0} \rightarrow \mathrm{K}^{-} \pi^{+}$ & $4.01 \pm 0.14$ & 0.0040 \\
\hline $\mathrm{D}^{+} \rightarrow \mathrm{K}^{-} \pi^{+} \pi^{+}$ & $9.1 \pm 0.6$ & 0.0091 \\
\hline
\end{tabular}

Table 3: Table of $\mathrm{D}$ meson decays most commonly used as flavour tags at LEP. All branching ratios are taken from the 1994 Review of Particle Properties [9]. For the number of decays per hadronic $\mathrm{Z}^{0}$, the predictions of the JETSET Monte Carlo program [4] are used for the partial widths and for the hadronisation fraction for a $\mathrm{D}$ to be produced, together with the branching ratios given.

\footnotetext{
${ }^{2}$ The following discussion is restricted to mesons, but is equally well applicable to baryons.
} 


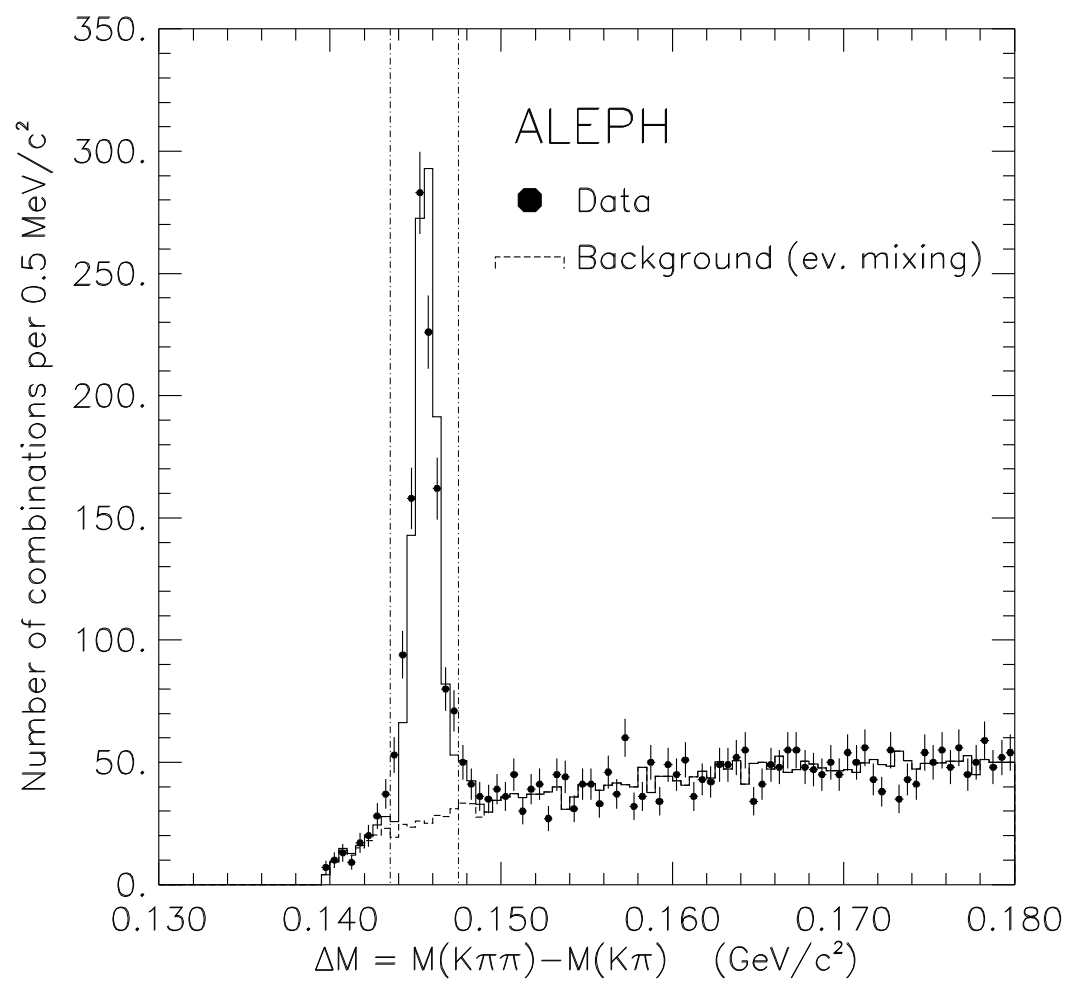

Figure 9: Distribution of the difference in invariant mass between a $\mathrm{D}^{*+}$ and a $\mathrm{D}^{0}$ candidate as reconstructed with the ALEPH detector [25]. Only candidates with a scaled energy larger than $25 \%$ of the beam energy are included in the plot.

\subsubsection{Reconstruction of D Mesons}

A large number of different $D$ states have been observed at LEP. The only ones currently interesting for use as tags for primary flavours are the $\mathrm{D}^{*+}$, and, to a lesser extent, the $\mathrm{D}$ meson ground states $\mathrm{D}^{+}$and $\mathrm{D}^{0}$. Analyses involving the $\mathrm{D}^{*+}$ meson have been published by ALEPH [25], OPAL [27] and DELPHI [31] . The $\mathrm{D}^{*+}$ is particularly noteworthy, because it always decays into a ground state $\mathrm{D}$ meson, $\mathrm{D}^{+}$or $\mathrm{D}^{0}$, with emission of either a pion or a photon. The masses of the $\mathrm{D}^{*+}$ and $\mathrm{D}^{0}$ differ only by $145.5 \mathrm{MeV}$, severely limiting the phasespace available to the decay products; in the $\mathrm{D}^{*+}$ rest-frame $40 \mathrm{MeV}$ are available in the decay $\mathrm{D}^{*+} \rightarrow \mathrm{D}^{0} \pi^{+}$. A very clean way of reconstructing this decay is to look at the mass difference between the $\mathrm{D}^{*+}$ and the $\mathrm{D}^{0}$ candidates. True $\mathrm{D}^{*+}$ decays show up in this plot as a narrow peak at $145.5 \mathrm{MeV}$, very close to the kinematic limit of $139.6 \mathrm{MeV}$, the pion mass. Such a mass difference plot is shown in figure 9, as reconstructed in the ALEPH detector. Clearly visible is the peak at $\Delta M=145.5 \mathrm{MeV}$, over very little background.

Other D meson decays do not profit from such a kinematical coincidence. Background levels, for example in the direct reconstruction of $\mathrm{D}^{+}$or $\mathrm{D}^{0}$ mesons, are therefore significantly higher.

The technical aspects of reconstructing a $\mathrm{D}^{*+}$ meson are well established, and are very similar amongst the different collaborations. After selecting hadronic events and good quality charged tracks, the $\mathrm{D}^{*+}$ reconstruction proceeds by first identifying a $\mathrm{D}^{0}$ candidate, then combining this candidate with a pion candidate to form the final $\mathrm{D}^{*+}$ candidate. Since all $\mathrm{D}^{0}$ decays 
contain at least one kaon, the particle identification power of the detectors is useful for $\mathrm{K}-\pi$ separation. OPAL and DELPHI rely on the measurement of the specific energy loss, $\mathrm{d} E / \mathrm{d} x$, in the central tracking chambers, for $\mathrm{K}$ identification. DELPHI also makes use of its ring imaging Cerenkov ("RICH") detector to clean up the sample in selected momentum ranges. Overall reconstruction efficiencies lie in the range 30 to $50 \%$ for $\mathrm{D}^{*+}$ which decay in this mode.

Samples of reconstructed $\mathrm{D}^{0}$ and $\mathrm{D}^{+}$mesons, selected without requiring that a $\mathrm{D}^{*+}$ be found, are much less clean and therefore more difficult to use as flavour tags. While for the $\mathrm{D}^{*+}$ mesons signal to background ratios of better than unity are achieved routinely, this reduces to 0.25 or worse for the more inclusive decays. Nevertheless some measurements can be made with these samples. The reconstruction proceeds as described above, with usually slightly tighter particle identification cuts, and some loose lifetime requirements. Analyses of the production properties of these D meson ground states have been published by ALEPH [25] and DELPHI [31].

\subsubsection{Flavour Separation in Reconstructed Particle Events}

Reconstructed D mesons need to be separated into $c \bar{c}$ and $b \bar{b}$ sources to make best use of them as $\mathrm{c}$ tags. Before attempting this separation, the light flavour background and bogus candidates in $\mathrm{b} \overline{\mathrm{b}}$ and $c \bar{c}$ events are subtracted statistically using the background to the specific D meson being investigated. Since the backgrounds are well known, this does not present any particular problems. The remaining sample is composed nearly exclusively of $c \bar{c}$ and $b \bar{b}$ events, which are separated in turn using $b$ tagging techniques. The $b$ tags were described in detail earlier in this review. The aspects special to analyses using reconstructed particles are discussed further here.

The separation usually proceeds by examining separately the two thrust hemispheres of the event, one of which contains the meson candidate. Flavour separation information can be obtained from either hemisphere. Use of the hemisphere opposite the meson tag provides a means of making a nearly unbiased separation, independent of the characteristics of the meson in the other hemisphere. Using the same hemisphere, on the other hand, although possibly biased towards the particular decay investigated, does allow an improvement of the separation power.

A number of techniques have been employed at LEP to separate the $c \bar{c}$ and $b \bar{b}$ contributions to D tagged events. Methods used rely on the lifetime of B mesons both in the same hemisphere in which the $\mathrm{D}$ meson has been reconstructed, and in the opposite hemisphere; on jet shape variables; on high momentum leptons; and on combinations of the previous techniques. ALEPH [25] have made the separation using event shapes, DELPHI [31] have used the D hemisphere lifetime, and OPAL have published an analysis combining all the methods [27].

Tagging techniques for charm differ in a number of important ways from those for bottom. Firstly, the number of c tagged events available from exclusive reconstruction is substantially smaller than the number selected with $b$ tags, because typical exclusive branching ratios are at most a few percent. At present electroweak measurements with $\mathrm{c}$ tags therefore suffer from very limited statistics. Secondly, since c tagged events are not positively tagged, but only statistically separated from b events, no individually $\mathrm{c}$ tagged events are available. No method yet allows a highly enriched $c \bar{c}$ sample to be prepared at LEP. 


\subsection{Hemisphere Charge Techniques for Estimating the Heavy Quark Charge}

While $\mathrm{D}^{*+}$ and lepton tags yield rather directly charge information about the decaying heavy quark from the charges of the identified particles, lifetime tags do not. In principle, the charges of the tracks associated to a reconstructed secondary vertex could be used to estimate the decaying hadron charge. Some progress has recently been reported on such a technique [32], but a problem remains that it is relatively rarely that exactly the correct tracks are associated with the secondary vertex. The impact parameter probability method of lifetime tagging also does not itself give any information about the decaying hadron charge.

To try to circumvent these difficulties, "jet charge" measures are used to estimate the primary quark charge from a weighted sum of the charges of several tracks in, for example, a thrust hemisphere. Jet charge definitions vary slightly, but the most commonly employed is:

$$
Q_{\text {hem }}=\frac{\Sigma_{i} q_{i}\left|p_{i}^{l}\right|^{\kappa}}{\Sigma_{i}\left|p_{i}^{l}\right|^{\kappa}}
$$

where the sum over $i$ runs over the tracks in the hemisphere, $q_{i}$ is the charge of track $i$, and $p_{i}^{l}$ is the component of its momentum along the thrust axis direction. The parameter $\kappa$ is chosen to give optimal charge sensitivity, typical values being $\kappa=0.5$ or $\kappa=0.7$. This type of hemisphere charge measure has been used in several analyses at LEP. So far the application to measurements of heavy flavour electroweak observables has been limited to the forward-backward asymmetry for lifetime-tagged $\mathrm{Z}^{0} \rightarrow \mathrm{b} \overline{\mathrm{b}}$ events (section 6 ) $[14,33]$.

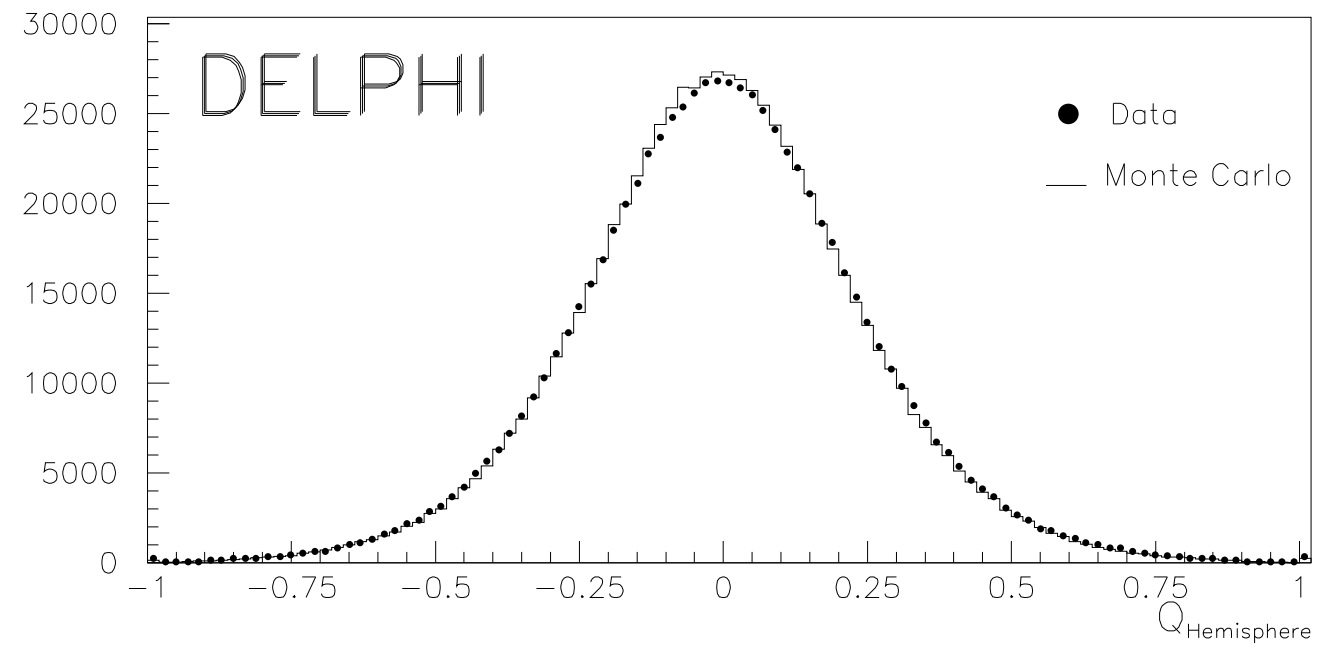

Figure 10: Hemisphere charge distribution observed by DELPHI [14]

The $Q_{\text {hem }}$ distribution observed by DELPHI [14] is shown in figure 10 . The shape of the distribution observed in the data is predicted quite well by the Monte Carlo. The measurements are rendered insensitive to the quality of this modelling, however, by measuring as far as possible the reliability of the jet charge estimator directly from the data.

ALEPH [33] use the mean difference of the charges measured in the thrust hemispheres to probe the forward-backward b asymmetry. The charge flow, $Q_{F B}$, is defined by:

$$
Q_{F B} \equiv Q_{F}-Q_{B}
$$


where $Q_{F}$ and $Q_{B}$ are the jet charges of the forward and backward thrust hemispheres, respectively. The distribution of the charge sum, $Q$, is also used:

$$
Q \equiv Q_{F}+Q_{B}
$$

These are illustrated schematically in figure 11 . The charge flow and charge sum distributions
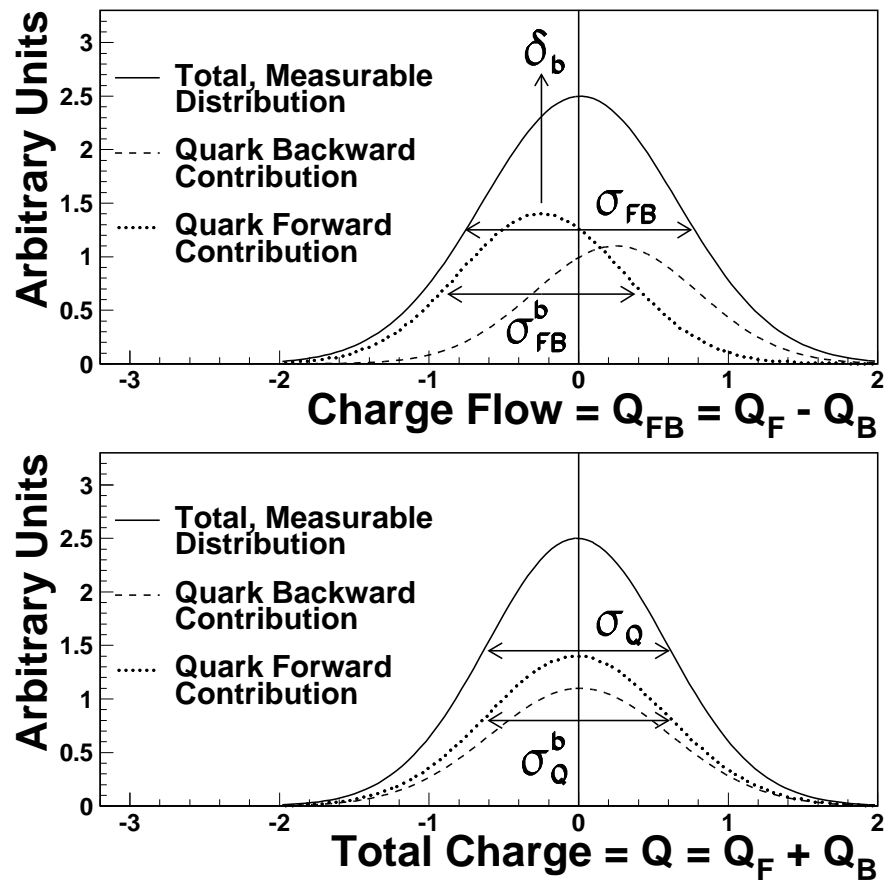

Figure 11: Schematic illustration [33] of the charge flow and total charge distributions for $b \bar{b}$ events.

are the sums of the two cases where the b quark was really produced in the forward or backward direction. In the absence of contamination by lighter quarks, the mean value of $Q_{F B}$ is simply related to the forward-backward asymmetry by:

$$
<Q_{F B}>=A_{\mathrm{FB}}^{\mathrm{b}} \delta
$$

where $\delta \equiv<Q_{\mathrm{b}}-Q_{\overline{\mathrm{b}}}>$, the charge separation, is the mean difference of the charges of the $\mathrm{b}$ and $\bar{b}$ hemispheres in $b \bar{b}$ events. The charge separation is not directly measurable, but can be inferred from the width of the charge sum and charge difference distributions with the addition of small corrections from Monte Carlo. The charge separations of light and c quark events are taken from Monte Carlo, but have little effect as they are heavily suppressed by the lifetime tag. The measurement of $\delta$ can be performed on the lifetime-tagged data sample itself, so that charge dilution from such effects as the time-dependence of $\mathrm{B}-\overline{\mathrm{B}}$ mixing are automatically taken into account.

DELPHI [14] use a simpler "event-by-event" approach to estimating the charge: the b quark hemisphere is taken to be the one with the lower jet charge. The probability of this assignment of hemispheres being incorrect is measured from samples of identified leptons. Selection of lepton samples with different $b$ purities allows cross-checks, as does separate use of electron and muon tags. The effect of mixing on the lepton side of the event is taken into account, as 
is the possible effect of time-dependent mixing on the lifetime-tagged side. The probability of correctly assigning the $\mathrm{b}$ quark and antiquark hemispheres is found to be $(67.3 \pm 1.2) \%$ [14], where the error includes a $\pm 0.5 \%$ contribution from the mixing uncertainty. As for the ALEPH method, the charge separations of light and c quark events are taken from Monte Carlo.

\section{Measurements of $\Gamma_{\mathrm{b} \overline{\mathrm{b}}} / \Gamma_{\mathrm{had}}$}

The first measurements of $\Gamma_{\mathrm{b}} / \Gamma_{\text {had }}$ from LEP primarily used "single-tag" techniques: i.e. events were examined, and if any tag were found in the event, the event was classified as tagged. The number of tagged events, $N_{\text {tag }}$, is then given by:

$$
N_{\text {tag }}=N_{\text {had }}\left(\varepsilon^{\mathrm{b}} \frac{\Gamma_{\mathrm{b} \overline{\mathrm{b}}}}{\Gamma_{\mathrm{had}}}+\varepsilon^{\mathrm{c}} \frac{\Gamma_{\mathrm{c} \overline{\mathbf{c}}}}{\Gamma_{\text {had }}}+\varepsilon^{\mathrm{u}} \frac{\Gamma_{\mathrm{u} \overline{\mathrm{u}}}}{\Gamma_{\text {had }}}+\varepsilon^{\mathrm{d}} \frac{\Gamma_{\mathrm{d} \overline{\mathrm{d}}}}{\Gamma_{\mathrm{had}}}+\varepsilon^{\mathrm{s}} \frac{\Gamma_{\mathrm{s} \overline{\mathrm{s}}}}{\Gamma_{\mathrm{had}}}\right)
$$

where $\varepsilon^{\mathrm{q}}$ is the probability of tagging an event where the $Z^{0}$ has decayed to $q \bar{q}$. For the tags used to date, the tagging efficiencies for $u \bar{u}$, $d \bar{d}$, and $s \bar{s}$ events are almost identical. Defining $\varepsilon^{\mathrm{uds}}$ to be the average tagging efficiency for these liqht quark events, and using the constraint

$$
\frac{\Gamma_{\mathrm{b} \overline{\mathrm{b}}}}{\Gamma_{\mathrm{had}}}+\frac{\Gamma_{\mathrm{c} \overline{\mathrm{c}}}}{\Gamma_{\mathrm{had}}}+\frac{\Gamma_{\mathrm{u} \overline{\mathrm{u}}}}{\Gamma_{\mathrm{had}}}+\frac{\Gamma_{\mathrm{d} \overline{\mathrm{d}}}}{\Gamma_{\text {had }}}+\frac{\Gamma_{\mathrm{s} \overline{\mathrm{s}}}}{\Gamma_{\mathrm{had}}}=1,
$$

equation (16) can be reformulated:

$$
N_{\mathrm{tag}}=N_{\text {had }}\left(\varepsilon^{\mathrm{b}} \frac{\Gamma_{\mathrm{b} \overline{\mathrm{b}}}}{\Gamma_{\text {had }}}+\varepsilon^{\mathbf{c}} \frac{\Gamma_{\overline{\mathbf{c}} \mathbf{c}}}{\Gamma_{\text {had }}}+\varepsilon^{\mathrm{uds}}\left(1-\frac{\Gamma_{\mathrm{b} \overline{\mathrm{b}}}}{\Gamma_{\text {had }}}-\frac{\Gamma_{\mathbf{c} \overline{\mathbf{c}}}}{\Gamma_{\text {had }}}\right)\right),
$$

giving for $\Gamma_{\mathrm{b} \overline{\mathrm{b}}} / \Gamma_{\mathrm{had}}$ :

$$
\frac{\Gamma_{\mathrm{b} \overline{\mathrm{b}}}}{\Gamma_{\mathrm{had}}}=\frac{\frac{N_{\mathrm{tag}}}{N_{\mathrm{had}}}-\left(\varepsilon^{\mathrm{c}}-\varepsilon^{\mathrm{uds}}\right) \frac{\Gamma_{\mathrm{c} \overline{\mathrm{c}}}}{\Gamma_{\mathrm{had}}}-\varepsilon^{\mathrm{uds}}}{\varepsilon^{\mathrm{b}}-\varepsilon^{\mathrm{uds}}} .
$$

Since $\varepsilon^{\mathrm{uds}}$ and $\varepsilon^{\mathrm{c}}$ are arranged to be much smaller than $\varepsilon^{\mathrm{b}}$, this reduces approximately to:

$$
\frac{\Gamma_{\mathrm{b} \overline{\mathrm{b}}}}{\Gamma_{\text {had }}} \approx \frac{N_{\text {tag }}}{\varepsilon^{\mathrm{b}} N_{\text {had }}} .
$$

In the single-tagging methods, $\varepsilon^{\mathrm{b}}$ is taken from a priori knowledge, via Monte Carlo and detector simulation programs. The statistical accuracy of this approach is limited by $N_{\text {tag }}$, and the systematic precision by how well $\varepsilon^{\mathrm{b}}$ is known. For typical event tagging efficiencies, $\varepsilon^{\mathrm{b}}$, of order $10 \%$, with order $\pm 10 \%$ relative uncertainty, this approach becomes systematics limited with only about 10000 hadronic $\mathrm{Z}^{0}$ decays.

Recent measurements of $\Gamma_{\mathrm{b}} / \Gamma_{\text {had }}$ have, therefore, used a different approach which does not require a priori knowledge of the b tagging efficiency - instead it is determined from the data themselves. This is achieved with the "double-tagging" approach. In this technique, the event is divided into two thrust hemispheres, and tagging algorithms are applied independently to each hemisphere in turn. The number of tagged hemispheres and the number of double-tagged events together allow both the $b$ tagging efficiency and $\Gamma_{\mathrm{b}} / \Gamma_{\text {had }}$ to be measured from the data. 
The number of tagged hemispheres, $N_{\mathrm{t}}$, can be written:

$$
N_{\mathrm{t}}=2 N_{\text {had }}\left(\varepsilon^{\mathrm{b}} \frac{\Gamma_{\mathrm{b} \overline{\mathrm{b}}}}{\Gamma_{\text {had }}}+\varepsilon^{\mathrm{c}} \frac{\Gamma_{\mathbf{c} \overline{\mathbf{c}}}}{\Gamma_{\text {had }}}+\varepsilon^{\mathrm{uds}}\left(1-\frac{\Gamma_{\mathrm{b} \overline{\mathrm{b}}}}{\Gamma_{\text {had }}}-\frac{\Gamma_{\mathbf{c} \overline{\mathbf{c}}}}{\Gamma_{\text {had }}}\right)\right)
$$

where it is assumed, as in equation (18), that the $\mathrm{u} \overline{\mathrm{u}}, \mathrm{d} \overline{\mathrm{d}}$ and $\mathrm{s} \overline{\mathrm{s}}$ efficiencies are the same, and that the $\mathrm{Z}^{0}$ hadronic decays are saturated by decays to the usual five quark flavours. The tagging efficiencies $\varepsilon^{\mathrm{b}}, \varepsilon^{\mathrm{c}}$ and $\varepsilon^{\mathrm{uds}}$ are here the hemispheric tagging probabilities. The number of double-tagged events, $N_{\mathrm{tt}}$, is then

$$
N_{\mathrm{tt}}=N_{\mathrm{had}}\left(C_{\mathrm{b}}\left(\varepsilon^{\mathrm{b}}\right)^{2} \frac{\Gamma_{\mathrm{b} \overline{\mathrm{b}}}}{\Gamma_{\mathrm{had}}}+C_{\mathrm{c}}\left(\varepsilon^{\mathrm{c}}\right)^{2} \frac{\Gamma_{\mathrm{c} \overline{\mathbf{c}}}}{\Gamma_{\mathrm{had}}}+C_{\mathrm{uds}}\left(\varepsilon^{\mathrm{uds}}\right)^{2}\left(1-\frac{\Gamma_{\mathrm{b} \overline{\mathrm{b}}}}{\Gamma_{\mathrm{had}}}-\frac{\Gamma_{\mathrm{c} \overline{\mathbf{c}}}}{\Gamma_{\mathrm{had}}}\right)\right)
$$

where the correlation parameters, $C_{\mathbf{q}}$, are introduced to take into account possible efficiency correlations between the two hemispheres. Equations (21) and (22) can be solved algebraically for $\Gamma_{\mathrm{b}} / \Gamma_{\text {had }}$ and $\varepsilon^{\mathrm{b}}$ if the other quantities are known from the data and simulation studies. Neglecting for clarity the background terms involving $\varepsilon^{\mathrm{c}}$ and $\varepsilon^{\mathrm{uds}}$, the solutions:

$$
\begin{aligned}
\frac{\Gamma_{\mathrm{b} \overline{\mathrm{b}}}}{\Gamma_{\mathrm{had}}} & \approx \frac{C_{\mathrm{b}} N_{\mathrm{t}}^{2}}{4 N_{\mathrm{tt}} N_{\mathrm{had}}} \\
\varepsilon^{\mathrm{b}} & \approx \frac{2 N_{\mathrm{tt}}}{C_{\mathrm{b}} N_{\mathrm{t}}}
\end{aligned}
$$

are obtained. The statistical disadvantage of the double-tagging method can be seen in these formulae: the statistical error on $\Gamma_{\mathrm{b}} / \Gamma_{\text {had }}$ is dominated by $N_{\mathrm{tt}}$, the number of double-tagged events. The main systematic uncertainties in the double tag approach come from knowledge of the correlation coefficient $C_{\mathrm{b}}$ and the background tagging efficiencies $\varepsilon^{\mathrm{c}}$ and $\varepsilon^{\text {uds }}$.

The correlation coefficient uncertainty is potentially rather serious, given the form of equation (23). The correlations arise from both physical and detector-related effects. Hard gluon radiation can give rise to correlations. In some cases, the gluon is very hard and recoils against the $b$ and $\bar{b}$ jets, which are found in the same thrust hemisphere. If the gluon is softer it can still give rise to $b$ and $\bar{b}$ momenta which are both lower than usual, giving lower tagging efficiency in the two hemispheres. On the detector side, tagging efficiencies which are not uniform over the geometrical acceptance of the detector can give rise to correlations in tagging efficiency. For lifetime tags, hemispheric correlations can also arise from the primary vertex position information, which is shared between the two hemispheres. In general, the effect of the correlation uncertainties is kept small by arranging the event selection so as to keep the correlating effects themselves small. Nonetheless, they give rise to a significant source of systematic uncertainty, as discussed below.

The background tagging efficiency uncertainties are the largest source of systematic error in the most precise $\Gamma_{\mathrm{b}} / \Gamma_{\text {had }}$ measurements available. Charmed hadron decay properties are most important, since $\mathrm{c}$ decays form the main background to b decays in all the tagging techniques. Detector resolution uncertainties also affect $\varepsilon^{\mathrm{c}}$ (and $\varepsilon^{\mathrm{uds}}$ ) and have to be treated carefully. In double lifetime tag measurements, the dominant background systematic errors come from the uncertainties on the $\mathrm{c}$ hadron mixtures, and on the mean $\mathrm{c}$ hadron charged decay multiplicity. A large uncertainty comes also from the relatively poorly-measured $\Gamma_{\mathbf{c} \overline{\mathbf{c}}} / \Gamma_{\mathrm{had}}$, unless the framework of the standard model is assumed, when the uncertainty is negligible since $\Gamma_{\mathbf{c} \overline{\mathbf{c}}} / \Gamma_{\text {had }}$ is quite precisely predicted. 
The measured value of $\Gamma_{\mathrm{b}} / \Gamma_{\text {had }}$ depends on the amount of gluon splitting to $\mathrm{b} \overline{\mathrm{b}}$ and $\mathrm{c} \overline{\mathrm{c}}$ pairs in the hadronisation process, because it can lead to b tags in light quark events. The level of gluon splitting has been calculated in perturbative QCD [34], with an estimated accuracy of $\pm 25-30 \%$. The analyses presented here take these heavy quarks from gluon splitting into account using the level predicted by the JETSET Monte Carlo, which is found to agree within $30 \%$ with the calculations. The errors quoted in table 4 correspond to varying the heavy quark production rate from gluon splitting by $\pm 50 \%$.

Published measurements of $\Gamma_{\mathrm{b}} / \Gamma_{\text {had }}$ are summarised in figure 12 . The average shown includes the results shown in the figure, taking into account common systematic errors between experiments [35]. Results shown in the figure are the quoted results from the original references $[10,13,15,18,19,26,28,36]$, which can assume different values of $\Gamma_{\mathbf{c} \bar{c}} / \Gamma_{\text {had }}$. Where the $\Gamma_{\mathbf{c} \overline{\mathbf{c}}} / \Gamma_{\text {had }}$ error was quoted as a separate error source, it has been excluded from the results shown. For the average, all results have been corrected to a common value of $\Gamma_{\mathrm{c}} / \Gamma_{\mathrm{had}}=0.171$, and no $\Gamma_{\mathbf{c} \overline{\mathbf{c}}} / \Gamma_{\text {had }}$ uncertainty included. Analyses from ALEPH and OPAL using lifetime information dominate the average. The OPAL "lifetime+lepton" measurement is a double-tag measurement which allows hemispheres to be tagged by either a secondary vertex tag or a lepton. An improved measurement using lifetime tags on a larger data sample has been performed by DELPHI [37], but has been finalised too late to be included in this review.

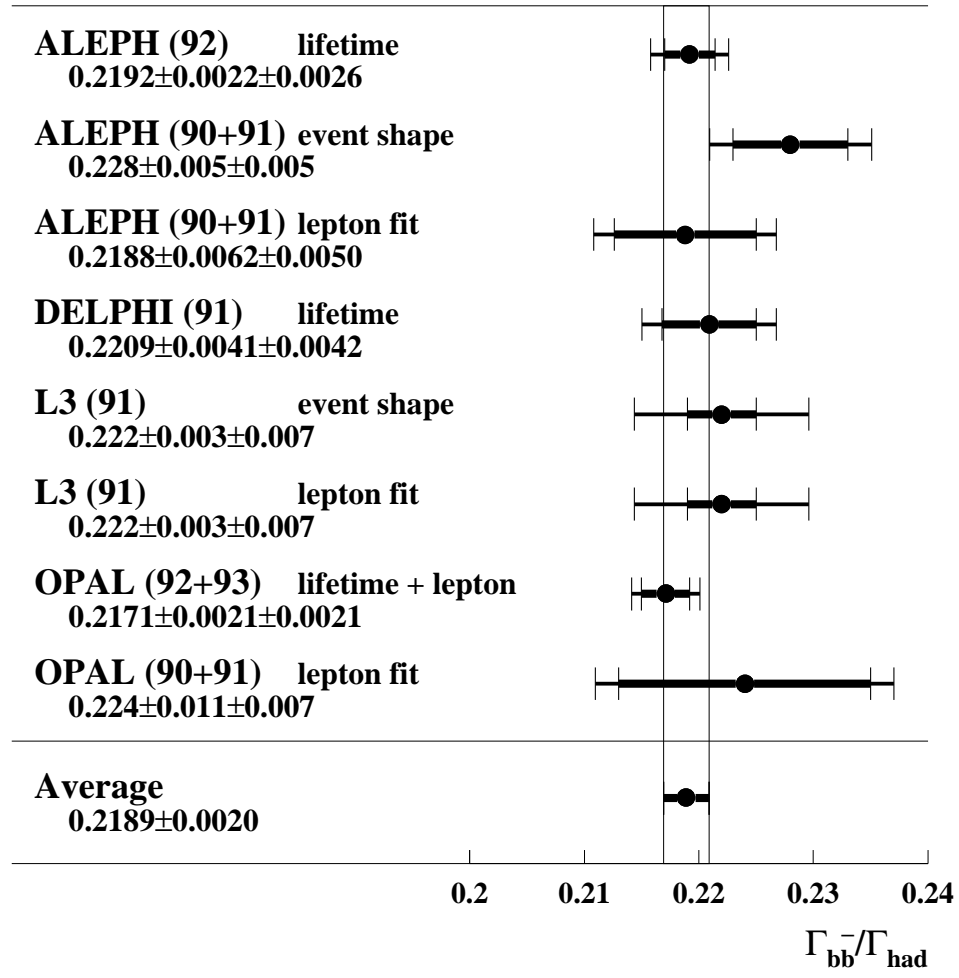

Figure 12: Published measurements of $\Gamma_{\mathrm{b} b} / \Gamma_{\text {had }}$ from LEP $[10,13,15,18,19,26,28,36]$. Numbers in brackets after collaboration names indicate the years in which the data analysed were collected. In this and subsequent plots of this type, the inner error bar indicates the statistical error, the outer error bar extends to cover the total error, including both statistical and systematic uncertainties.

The improvement in accuracy in measurements of $\Gamma_{\mathrm{b} \overline{\mathrm{b}}} / \Gamma_{\text {had }}$ is illustrated in figure 13. Earlier 


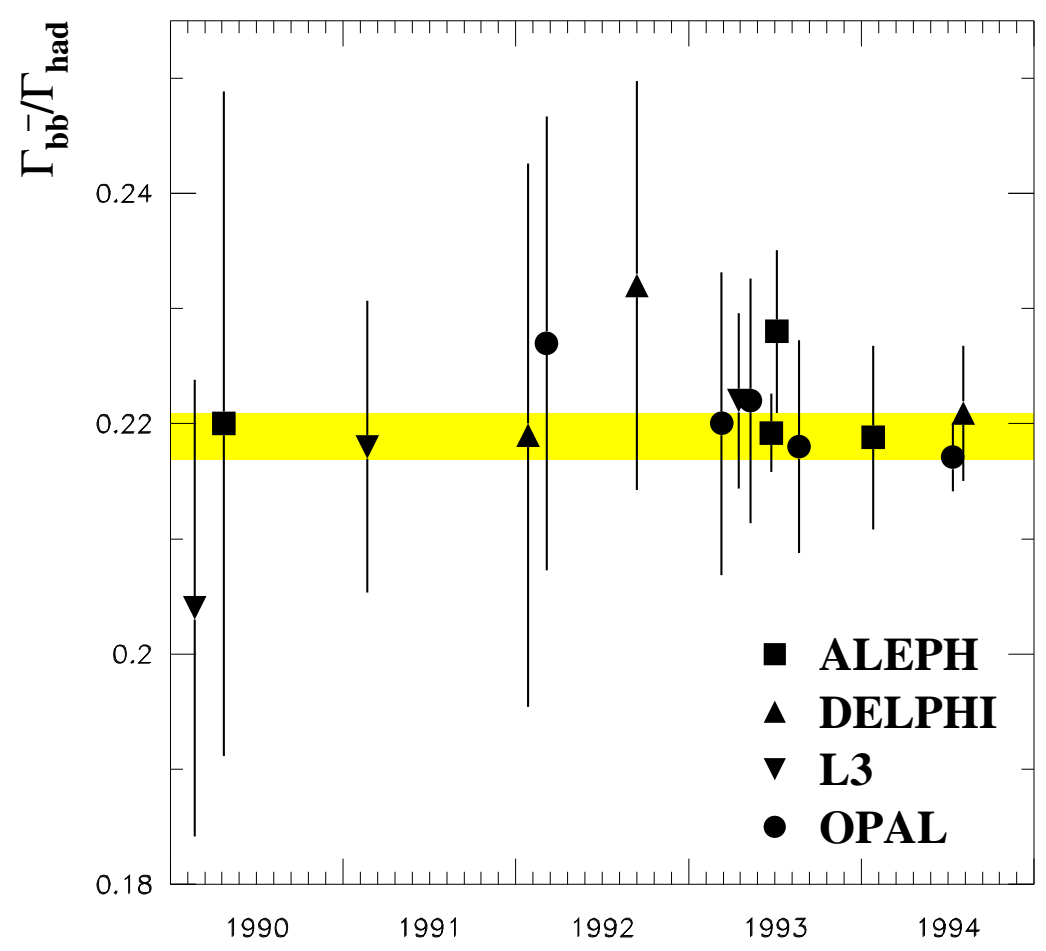

Date

Figure 13: The evolution of measurements of $\Gamma_{\mathrm{b}} / \Gamma_{\mathrm{h} a d}$ with time. The date indicated is that of submission to the journal.

measurements were primarily of the single-tag type, while later, more accurate, measurements are all of the double-tag type. All measurements included in figure 12 use either double tagging as described here, or lepton fits which also use doubly lepton-tagged events, as described in section 3.2.

Figure 12 indicates that the systematic errors in the measurement of $\Gamma_{\mathrm{b}} \overline{\mathrm{b}} / \Gamma_{\text {had }}$ are of a similar size to the statistical errors. A more detailed error breakdown is shown in table 4 for the precise ALEPH and OPAL measurements. The main sources of systematic errors are those due to the charm background contamination and the tracking resolution with a significant contribution from tagging efficiency correlations between the two event hemispheres. Comparing the two analyses, it is noticeable that the charm background errors are larger for the OPAL analysis, the tracking resolution errors for the ALEPH analysis. The former effect arises from the lower purity of the $b$ tag used by OPAL compared to that of ALEPH (see table 2 above), and because addition of lepton tags by OPAL also increases the charm contamination a little. The OPAL tracking resolution error is relatively low because of the use of a new technique, "folded double tagging".

In the folded double tag approach [15], the number of hemispheres with a reconstructed secondary vertex with a negative decay length significance is subtracted from the number with a positive significance. The "forward" tagging requirement used in the analysis, $L / \sigma>8$, is thus mirrored by a "backward tag" of hemispheres with a vertex with $L / \sigma<-8$ (see figure 4 ). The algebra of the double-tagging equations 21 and 22 is not modified if the substitutions:

$$
N_{\mathrm{t}}=N_{\mathrm{v}}-N_{\overline{\mathrm{v}}}
$$




\begin{tabular}{|l|c|c|}
\hline Error Source & ALEPH $[10]$ & OPAL $[15]$ \\
\hline \hline Detector/Simulation Effects & & \\
Tracking resolution & 0.0014 & 0.0007 \\
Lepton efficiency and backgrounds & - & 0.0006 \\
Monte Carlo statistics & 0.0014 & 0.0005 \\
Event selection flavour bias & 0.0003 & 0.0003 \\
Charm Background & & \\
c hadron production fractions & 0.0009 & 0.0009 \\
c hadron lifetimes & 0.0005 & 0.0004 \\
c hadron decay properties & 0.0006 & 0.0010 \\
c fragmentation & 0.0001 & 0.0007 \\
Light Quark Background & & \\
$g \rightarrow$ c/ $/$ b & 0.0004 & 0.0005 \\
K, hyperon production rates & 0.0003 & 0.0003 \\
Hemisphere Correlations & 0.0009 & 0.0007 \\
\hline Total systematical & 0.0026 & 0.0021 \\
Statistical & 0.0022 & 0.0021 \\
\hline
\end{tabular}

Table 4: Breakdown of error sources for the two currently most precise measurements of $\Gamma_{\mathrm{b}} / \Gamma_{\mathrm{had}}$.

$$
N_{\mathrm{tt}}=N_{\mathrm{vv}}-N_{\mathrm{v} \overline{\mathrm{v}}}+N_{\overline{\mathrm{vv}}}
$$

are made, where $N_{\mathrm{v}}$ is the number of forward tagged hemispheres, $N_{\overline{\mathrm{v}}}$ the number of backward tagged hemispheres, $N_{\mathrm{vv}}$ the number of events with both hemispheres forward tagged, $N_{\mathrm{v} \overline{\mathrm{v}}}$ the number of events with one forward and one backward tagged hemisphere, and $N_{\overline{\mathrm{vv}}}$ the number with both hemispheres backward tagged. This statistical subtraction reduces significantly the sensitivity of the result to resolution uncertainties, at the cost of an increase in statistical error. In practice, the number of backward tagged hemispheres is approximately $4 \%$ of the number of forward tagged hemispheres, so the loss in statistical precision is unimportant.

The fraction of hadronic $\mathrm{Z}^{0}$ decays to $\mathrm{b} \overline{\mathrm{b}}$ pairs is not expected to vary by more than $1 \%$ over the centre-of-mass energy range probed so far at LEP (88-95 GeV). Since the majority of the data are collected at the peak energy and very little are collected at the extreme energies, the effect on the measurements can be expected to be very small. Several $\Gamma_{\mathrm{b}} / \Gamma_{\text {had }}$ measurements tacitly assume this, since they average data from the different energy points, while others use only data taken at the peak energy. OPAL [15] and DELPHI [13] have explicitly checked that no variation is observed, although the precision of the test is at the $3-5 \%$ level.

Combining the individual $\Gamma_{\mathrm{b}} / \Gamma_{\text {had }}$ measurements in figure 12, taking into account common systematic errors between the different measurements as discussed in section 7 below, an average LEP value of

$$
\frac{\Gamma_{\mathrm{b} \overline{\mathrm{b}}}}{\Gamma_{\mathrm{had}}}=0.2189 \pm 0.0020
$$

is obtained, for $\Gamma_{\mathbf{c} \overline{\mathbf{c}}} / \Gamma_{\text {had }}=0.171$, where the total error quoted includes both statistical and systematic errors. The dependence of this result on $\Gamma_{\overline{\mathbf{c}}} / \Gamma_{\text {had }}$, and its consistency with the standard model, is discussed in section 7. The prospects for improving the measurement in future are discussed in section 9 . The ratio $\Gamma_{\mathrm{b}} / \Gamma_{\text {had }}$ has also been measured at the SLC linear 
collider. The most precise published measurement from SLC [11] is $\Gamma_{\mathrm{b} \overline{\mathrm{b}}} / \Gamma_{\text {had }}=0.251 \pm 0.049 \pm$ 0.030 , consistent with, but much less precise than, the LEP average.

\section{Measurements of $\Gamma_{\mathrm{c} \bar{c}} / \Gamma_{\text {had }}$}

The number of tagged $c \bar{c}$ events available at LEP is significantly smaller than the number of $\mathrm{b} \overline{\mathrm{b}}$ events. Therefore measurements of the partial width $\Gamma_{\mathbf{c} \overline{\mathbf{c}}} / \Gamma_{\text {had }}$ are considerably less precise than equivalent measurements in the b sector, with measurements still relying on the single tag technique.

Measurements of $\Gamma_{\mathbf{c} \bar{c}} / \Gamma_{\text {had }}$ have been performed for a number of different $\mathrm{c}$ tagging methods. The first measurements of $\Gamma_{\mathbf{c} \overline{\mathbf{c}}} / \Gamma_{\text {had }}$ used an inclusive lepton tag in a manner equivalent to the $\mathrm{b}$ tagging method [38], but to measure $\mathrm{c}$ quark properties. When fitting the $p$ and $p_{t}$ spectra of the lepton candidates, both $\mathrm{b}$ and $\mathrm{c}$ components are allowed to float, resulting in a simultaneous measurement of $\Gamma_{\mathrm{b}} / \Gamma_{\text {had }}$ and $\Gamma_{\mathbf{c} \bar{c}} / \Gamma_{\text {had }}$. Relatively large systematic uncertainties arise for $\Gamma_{\mathbf{c} \bar{c}} / \Gamma_{\text {had }}$. Since the rate of $\mathbf{c}$ quark production is derived from the shape of the $p$ and $p_{t}$ spectra, and since charm and background leptons have similar distributions, the result depends rather critically on the assumed spectral shapes. In addition these analyses are targeted more at measuring $\Gamma_{\mathrm{b}} / \Gamma_{\text {had }}$, so the c sensitivity is not optimal. Nevertheless competitive measurements have been made using this method.

Another early analysis at LEP was published in 1990 [39] and used the small momentum available to the transition pion in the decay $\mathrm{D}^{*+} \rightarrow \mathrm{D}^{0} \pi$ as an inclusive tag for charm. Looking at the transverse momentum spectrum of all tracks in a hemisphere, a peak is expected and observed for $c \bar{c}$ events at very low values of $p_{t}$. Very few processes exist which can produce an excess at these low transverse momentum values. However the background from fragmentation tracks is very large. This measurement therefore suffers from difficult systematic errors, and has not been repeated.

Attempts have been made to measure $\Gamma_{\mathbf{c} \bar{c}} / \Gamma_{\text {had }}$ using the jet-shape variables discussed above. The DELPHI collaboration [30] has published such an analysis, with, however, large systematic errors from the modelling of the network response by the Monte Carlo.

Several measurements employ D mesons as reconstructed particle tags, including the most precise single measurement to date. DELPHI [31] have presented results using $\mathrm{D}^{0}$ and $\mathrm{D}^{ \pm}$ rates. Both the $\mathrm{D}^{0} \rightarrow \mathrm{K}^{-} \pi$ and the $\mathrm{D}^{+} \rightarrow \mathrm{K}^{-} \pi^{+} \pi^{+}$decays are identified, and are separated into components from charm and from bottom using lifetime information, so allowing the product $\Gamma_{\mathbf{c} \overline{\mathbf{c}}} / \Gamma_{\text {had }} P_{c}$ to be determined. Here $P_{c}$ is the product branching ratio that a charm quark fragments into the $\mathrm{D}$ meson investigated and then decays in the channel in question. $P_{c}$ is taken from lower energy measurements, to derive $\Gamma_{\mathbf{c} \bar{c}} / \Gamma_{\text {had }}$. Since all D mesons eventually decay into one of these ground state mesons, this method does not depend severely on assumptions about the relative production fraction of a particular D meson in charm fragmentation. OPAL [27], on the other hand, rely on the assumption that the $\mathrm{D}^{*+}$ meson is produced in the same way at LEP energies as it is in lower energy $\mathrm{e}^{+} \mathrm{e}^{-}$collisions. This allows the determination of $\Gamma_{\mathbf{c} \overline{\mathbf{c}}} / \Gamma_{\text {had }}$ from the measured rate of $\mathrm{D}^{*+}$ production, which has been measured with a smaller error than the $\mathrm{D}^{0}$ or $\mathrm{D}^{+}$production rate. Again lower energy measurements are used to determine a value for the product branching ratio $B\left(\mathrm{c} \rightarrow \mathrm{D}^{*+} X\right) B\left(\mathrm{D}^{*+} \rightarrow \mathrm{K} \pi \pi\right)$.

The currently available results for $\Gamma_{\mathbf{c} \overline{\mathbf{c}}} / \Gamma_{\text {had }}$ are summarised in figure 14 . Combining all, 


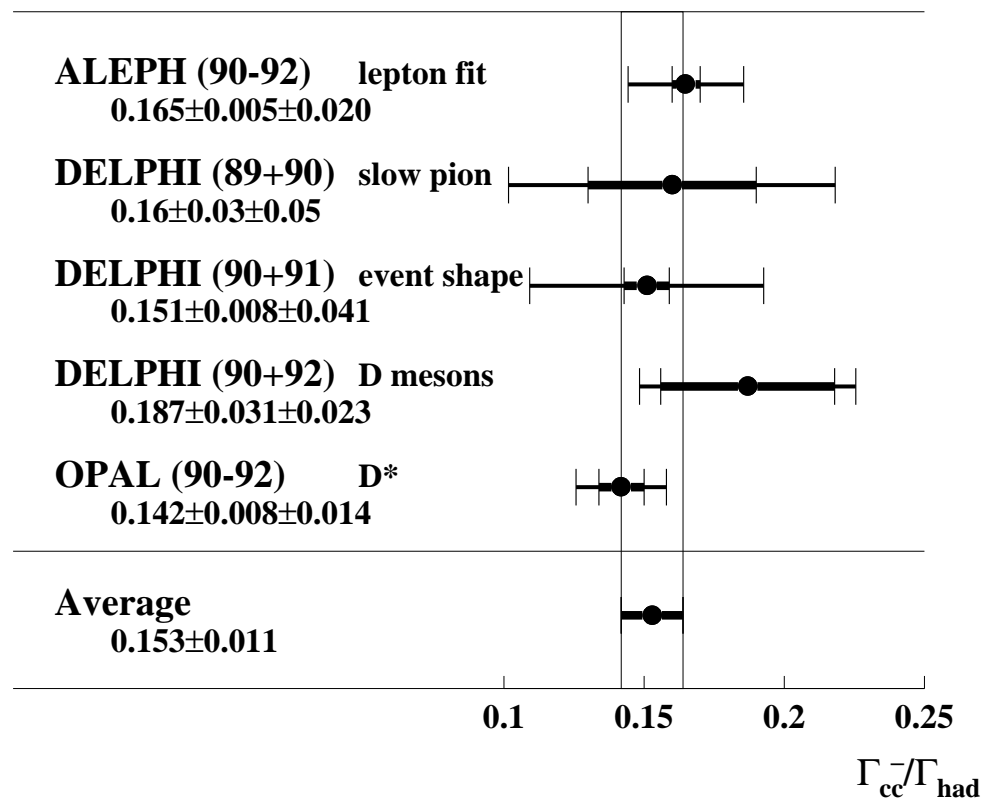

Figure 14: Compilation of current published measurements of $\Gamma_{\mathrm{c} \bar{c}} / \Gamma_{\text {had }}[27,30,31,38,39]$. The individual values are the ones quoted in the appropriate papers. For the average, correlations between the measurements have been taken into account.

following the procedure described in section 7, an average LEP value of

$$
\Gamma_{\mathbf{c} \overline{\mathbf{c}}} / \Gamma_{\text {had }}=0.155 \pm 0.011
$$

is found, for $\Gamma_{\mathrm{b}} / \Gamma_{\text {had }}=0.217$. The dependence of this result on $\Gamma_{\mathrm{b} \overline{\mathrm{b}}} / \Gamma_{\mathrm{had}}$, and its consistency with the standard model prediction, is discussed in section 7 .

The time evolution of the precision of published $\Gamma_{\mathbf{c} \overline{\mathbf{c}}} / \Gamma_{\text {had }}$ measurements is shown in figure 15 . A gradual improvement with time is evident.

The most precise $\Gamma_{\mathbf{c} \bar{c}} / \Gamma_{\text {had }}$ measurements are now systematics limited. The most accurate uses $\mathrm{D}^{*+}$ mesons as charm tags and is limited by the poor knowledge of the hadronisation fraction $B\left(\mathrm{c} \rightarrow \mathrm{D}^{*+} X\right)$, which is not very amenable to improvement. The prospects of improving the overall precision on $\Gamma_{\mathbf{c} \bar{c}} / \Gamma_{\text {had }}$ in future are discussed in section 9 .

\section{Measurements of Forward-Backward Asymmetries}

Measurement of the forward-backward asymmetry of b or c quarks requires three things: a tagging technique must be used to enhance the contribution of the appropriate heavy flavour; an estimator of the direction of the heavy quark or antiquark is needed; and a charge estimator has to be derived to separate the quark direction from that of the antiquark.

Lepton tags provide both heavy flavour tags, and, through their charge, information about whether the decaying heavy hadron contains a heavy quark or antiquark. Reconstructed particle tags also conveniently provide directly the quark/antiquark separation. Lifetime tags do not provide such charge information, but the hemisphere charge measurement described in section 3.5 can be used instead. 


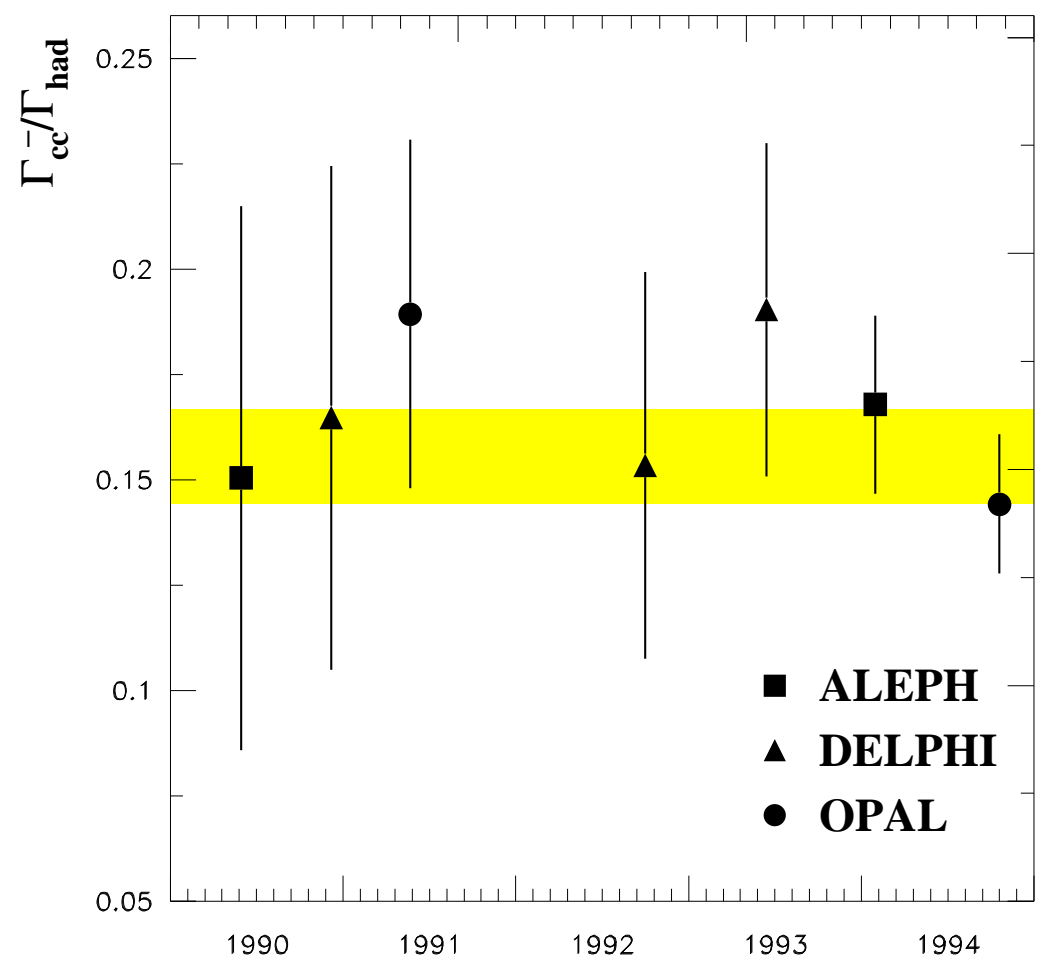

Date

Figure 15: The evolution of measurements of $\Gamma_{\mathrm{c} \bar{c}} / \Gamma_{\text {had }}$ with time. The date indicated is that of submission to the journal.

For lepton and $\mathrm{D}^{*+}$ tags, an experimentally measurable variable $y$ is usually constructed to estimate the outgoing heavy quark direction:

$$
y \equiv \pm Q \cos \theta_{\text {thrust }}
$$

where $Q$ is the charge of the tag, and $\cos \theta_{\text {thrust }}$ is the polar angle of the thrust axis signed to point into the same thrust hemisphere as the jet containing the tag. For lepton tags, the negative sign is adopted, for $\mathrm{D}^{*+}$ tags the positive sign. In the $\mathrm{D}^{*+}$ tag case, $y$ estimates correctly both $\mathrm{b}$ and $\mathrm{c}$ quark directions, since both quarks give rise to positively charged $\mathrm{D}^{*+}$ mesons. In the lepton case, the direction of semileptonically decaying $b$ quarks is correctly estimated by $y$ because such decays give rise to negatively charged leptons, but the sign is flipped for $c \bar{c}$ events because semileptonic c decays give positive leptons. The data are assumed to follow a functional form

$$
\frac{\mathrm{d} \sigma}{\mathrm{d} y} \propto 1+y^{2}+\frac{8}{3} A_{\mathrm{FB}}^{\mathrm{meas}} y
$$

with a similar structure to equation (6) in section 2. The overall asymmetry measured in a sample of tagged events, $A_{\mathrm{FB}}^{\text {meas }}$, has the form

$$
A_{\mathrm{FB}}^{\text {meas }}=g_{\mathrm{b}-\text { tagged }} A_{\mathrm{FB}}^{\mathrm{b}}+g_{\mathrm{c}-\text { tagged }} A_{\mathrm{FB}}^{\mathrm{c}}+g_{\mathrm{bkgd}} A_{\mathrm{FB}}^{\mathrm{bkgd}},
$$

where the $g$ are appropriate factors, including the fractions of each source in the sample being fit and corrections for effects such as B-B mixing. Note that in the case of lepton tagged c $\overline{\mathrm{c}}$ events, for example, $g_{c \text {-tagged }}$ is negative, because of the decay lepton is positively charged. 
The data are fit to the shape of equation (27), using either an unbinned log-likelihood fit or a binned chi-squared technique, or, in the case of the lepton analysis of L3, a more sophisticated unbinned technique. Instrumental background may first be subtracted from the sample, in which case $g_{b k g d}$ in equation (28) is set to zero. A different fit has also been used [14,40], where instead of fitting the full $y$ distribution to the form of equation 27 , a fit is made using the ratio

$$
\left(N^{\mathrm{F}}-N^{\mathrm{B}}\right) /\left(N^{\mathrm{F}}+N^{\mathrm{B}}\right)
$$

in bins of $|y|$, where $N^{\mathrm{F}}$ and $N^{\mathrm{B}}$ are the numbers of tags with $y>0$ and $y<0$ respectively, in each $|y|$ bin. The distribution of this variable follows

$$
\frac{8}{3} A_{\mathrm{FB}}^{\text {meas }} \frac{y}{1+y^{2}}
$$

for $y>0$. In both this fit and the unbinned log-likelihood method, the variation of the identification efficiency with $\cos \theta_{\text {thrust }}$ does not need to be known - only the relative efficiencies of the different sources are needed.

Systematic errors could potentially arise if the detection efficiencies for differently charged tags were different, by different amounts in the forward and backward regions of the detector. Such effects can be studied directly using the data, and no significant effect has been found by any experiment.

\subsection{Asymmetry Measurements with Leptons}

In a semileptonic $\mathrm{b} \rightarrow \ell^{-}$decay, the lepton charge accurately reflects the charge of the decaying $b$ quark. Experimentally, any sample of selected leptons also contains decays such as $\mathrm{b} \rightarrow \mathrm{c} \rightarrow \ell^{+}$, $\mathrm{b} \rightarrow \overline{\mathrm{c}} \rightarrow \ell^{-}, \mathrm{c} \rightarrow \ell^{+}$, and so on, which affect the measured forward-backward asymmetry. In addition, the forward-backward asymmetry of decaying $\mathrm{b}$ hadrons is diluted from that of the produced hadrons because of the effect of $\mathrm{B}-\overline{\mathrm{B}}$ mixing. The overall asymmetry, $A_{\mathrm{FB}}^{\text {meas }}$, in a sample of identified leptons can be written:

$$
A_{\mathrm{FB}}^{\mathrm{meas}}=\left(f_{\mathrm{b} \rightarrow \ell^{-}}+f_{\mathrm{b} \rightarrow \tau \rightarrow \ell^{-}}-f_{\mathrm{b} \rightarrow \mathrm{c} \rightarrow \ell^{+}}+f_{\mathrm{b} \rightarrow \overline{\mathrm{c}} \rightarrow \ell^{-}}\right)(1-2 \chi) A_{\mathrm{FB}}^{\mathrm{b}}-f_{\mathrm{c} \rightarrow \ell^{+}} A_{\mathrm{FB}}^{\mathrm{c}}+f_{\mathrm{bkgd}} A_{\mathrm{FB}}^{\mathrm{bkgd}}
$$

where $f_{i}$ is the fraction of leptons from source $i$ in the sample, $\chi$ is the average $\mathrm{B}-\overline{\mathrm{B}}$ mixing in the lepton sample, and $f_{\mathrm{bkgd}}$ and $A_{\mathrm{FB}}^{\mathrm{bkgd}}$ refer to instrumental backgrounds.

As discussed in section 3.2, lepton samples are used to tag heavy flavours either by making hard $p$ and $p_{t}$ cuts to isolate a nearly pure b sample, or by fitting for the contributions of the different lepton sources in a more inclusive sample. This latter approach has been pursued very productively in the case of asymmetry measurements as it allows $A_{\mathrm{FB}}^{\mathrm{b}}, A_{\mathrm{FB}}^{\mathrm{c}}$ and/or $\chi$ to be measured simultaneously. If in addition some of the various semileptonic decay branching ratios are allowed to vary in the fit, reduced systematic uncertainties on the asymmetries can be obtained.

Example results of the asymmetry measurements with leptons are shown in figures 16 and 17. All the LEP published results for the $b \bar{b}$ and $c \bar{c}$ forward-backward asymmetries measured with lepton fits are shown in table 5. Results for data taken at centre-of-mass energies corresponding to the sides of the peak of the $Z^{0}$ resonance are summarised in table 10 below.

The main systematic errors on the measurements arise from knowledge of the lepton backgrounds and the relative fractions $f_{i}$ of leptons from the different sources in the sample. Table 5 

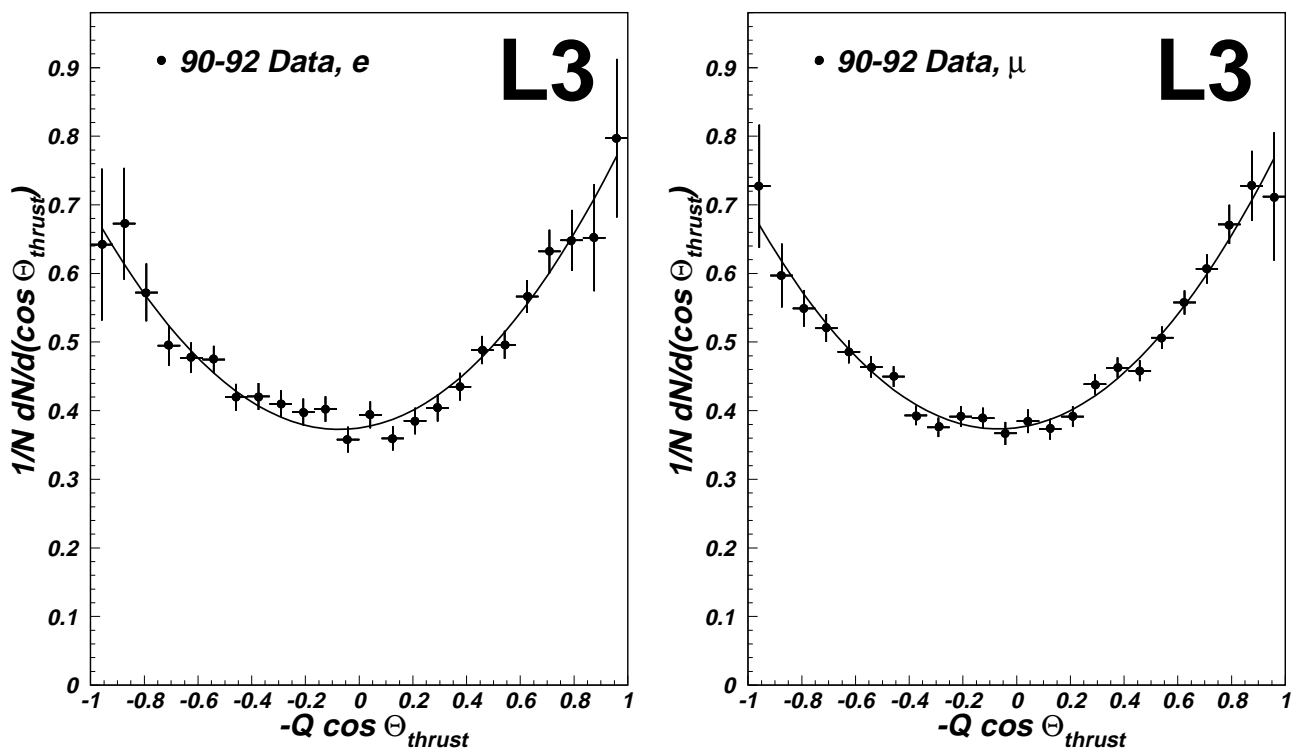

Figure 16: The measured distribution of $-Q \cos \theta_{\text {thrust }}$ for electrons (left) and muons (right) with $p_{t}>1 \mathrm{GeV}$, as observed by L3 [17]. The distributions are corrected for acceptance and instrumental backgrounds are subtracted. The curve shows the fitted value.
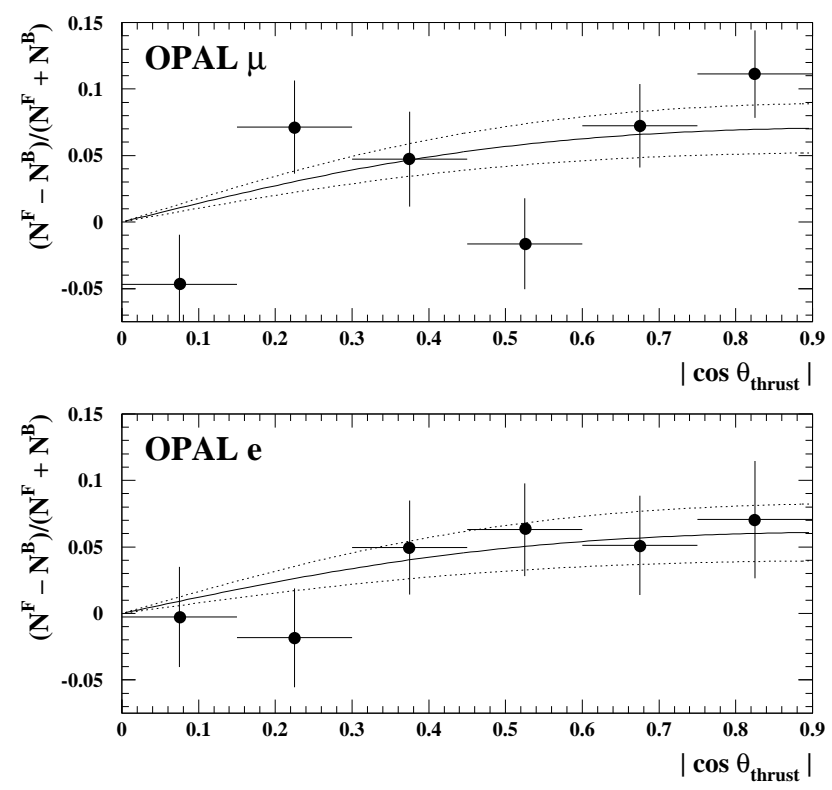

Figure 17: The measured distribution of $\left(N^{\mathrm{F}}-N^{\mathrm{B}}\right) /\left(N^{\mathrm{F}}+N^{\mathrm{B}}\right)$ obtained by OPAL [40] for muons (top) and electrons (bottom) with $p>3 \mathrm{GeV}$ and $p_{t}>1 \mathrm{GeV}$ (muons) or $p>2 \mathrm{GeV}$ and $p_{t}>0.8 \mathrm{GeV}$ (electrons). The solid curve shows the best fit value, the dashed curves the one standard deviation variation for the fitted asymmetry.

indicates clearly that the $A_{\mathrm{FB}}^{\mathrm{b}}$ measurements are strongly statistics limited, and there is scope for sizeable reductions in the dominant experimental systematic errors. These are often limited by the statistics of control data samples. Measurements of $A_{\mathrm{FB}}^{\mathrm{c}}$, on the other hand, have comparable statistical and systematic errors. Again in this case, the experimental systematic 


\begin{tabular}{|c|c|c|}
\hline & $A_{\mathrm{FB}}^{\mathrm{b}}$ & $A_{\mathrm{FB}}^{\mathrm{c}}$ \\
\hline ALEPH [19] & $0.087 \pm \mathbf{0 . 0 1 4} \pm \mathbf{0 . 0 0 3}$ & $0.099 \pm 0.020 \pm 0.018$ \\
\hline DELPHI [14] & $0.104 \pm 0.013 \pm 0.005$ & $0.083 \pm 0.022 \pm 0.016$ \\
\hline L3 $[17,41](*)$ & $0.087 \pm 0.011 \pm 0.004$ & $0.083 \pm 0.038 \pm 0.027$ \\
\hline OPAL [40] & $0.092 \pm 0.018 \pm 0.008$ & $0.014 \pm 0.030 \pm 0.020$ \\
\hline
\end{tabular}

Table 5: Current published results for $A_{\mathrm{FB}}^{\mathrm{b}}$ and $A_{\mathrm{FB}}^{\mathrm{c}}$ from lepton fits. Only results from centre-ofmass energies within $200 \mathrm{MeV}$ of the $\mathrm{Z}^{0}$ mass are shown ("on-peak" data). The first error shown in each case is statistical, the second systematic. The results are the fitted values of $A_{\mathrm{FB}}^{\mathrm{b}}$ and $A_{\mathrm{FB}}^{\mathrm{c}}$ for $\mathrm{Z}^{0}$ decays in $\mathrm{e}^{+} \mathrm{e}^{-}$collisions. Corrections needed to convert these values into pole asymmetries have not been applied. Note $\left({ }^{*}\right)$ that the L3 results for $A_{\mathrm{FB}}^{\mathrm{b}}$ and $A_{\mathrm{FB}}^{\mathrm{c}}$ were not derived from the same data sample.

errors are amenable to reduction as further data are analysed. Systematic uncertainties from semileptonic decay models and branching ratio assumptions are significant in the $A_{\mathrm{FB}}^{\mathrm{c}}$ measurement, however, and these will be more difficult to reduce. Some progress will be possible by fitting for the branching ratios in the lepton samples themselves, in those cases where this is not already done.

\subsection{Asymmetry Measurements with Reconstructed Particles}

A complementary method of measuring the forward-backward asymmetries uses reconstructed $\mathrm{D}^{*+}$ mesons to tag $\mathrm{b} \overline{\mathrm{b}}$ and $c \bar{c}$ events. Unlike the lepton tags discussed above, this method is more sensitive to the $c$ asymmetry than to that of the $b$. Such analyses have been presented by ALEPH, DELPHI and OPAL $[25,42,43]$.

Details of the reconstruction of charmed mesons were discussed in section 3.4. The very low tagging efficiencies obtainable with reconstructed particles mean that the asymmetry measurements made with them are heavily statistics limited. The overall precision of the measurement can be improved by including many different decays, even in channels with relatively high backgrounds.

At present only the $\mathrm{D}^{*+}$ meson has been used as a tag particle for published asymmetry measurements. Three different decay modes in the subsequent decay of the $\mathrm{D}^{0}$ are employed:

$$
\begin{aligned}
\mathrm{D}^{*+} \rightarrow & \mathrm{D}^{0} \pi^{+} \\
& \hookrightarrow \mathrm{K}^{-} \pi^{+} \\
& \hookrightarrow \mathrm{K}^{-} \pi^{+} \pi^{-} \pi^{+} \\
& \hookrightarrow \mathrm{K}^{-} \pi^{+} \pi^{0}
\end{aligned}
$$

The $\pi^{0}$ in decay mode (iii) need not be reconstructed. A large fraction of the $\mathrm{D}^{0}$ decays in this channel proceed through a $\rho^{+}$, resulting in an enhancement in the K- $\pi$ invariant mass spectrum around $1.6 \mathrm{GeV}$. The width of this peak is considerably larger than the fully reconstructed mass peaks in the other channels. The invariant mass spectrum for $\mathrm{D}^{0}$ candidates as found with the DELPHI detector is shown in figure 18. Clearly visible are the main peak from process (i) and the satellite peak from process (iii).

The asymmetry is fit to equation (27), with the overall measured asymmetry given by

$$
A_{\mathrm{FB}}^{\text {meas }}=\left(f_{\mathrm{b} \rightarrow \mathrm{D}^{*+}}-f_{\mathrm{b} \rightarrow \mathrm{D}^{*-}}\right)\left(1-2 \chi_{\mathrm{D}^{*+}}\right) A_{\mathrm{FB}}^{\mathrm{b}}+f_{\mathrm{c} \rightarrow \mathrm{D}^{*+}} A_{\mathrm{FB}}^{\mathrm{c}}+f_{\mathrm{bkgd}} A_{\mathrm{FB}}^{\mathrm{bkgd}},
$$




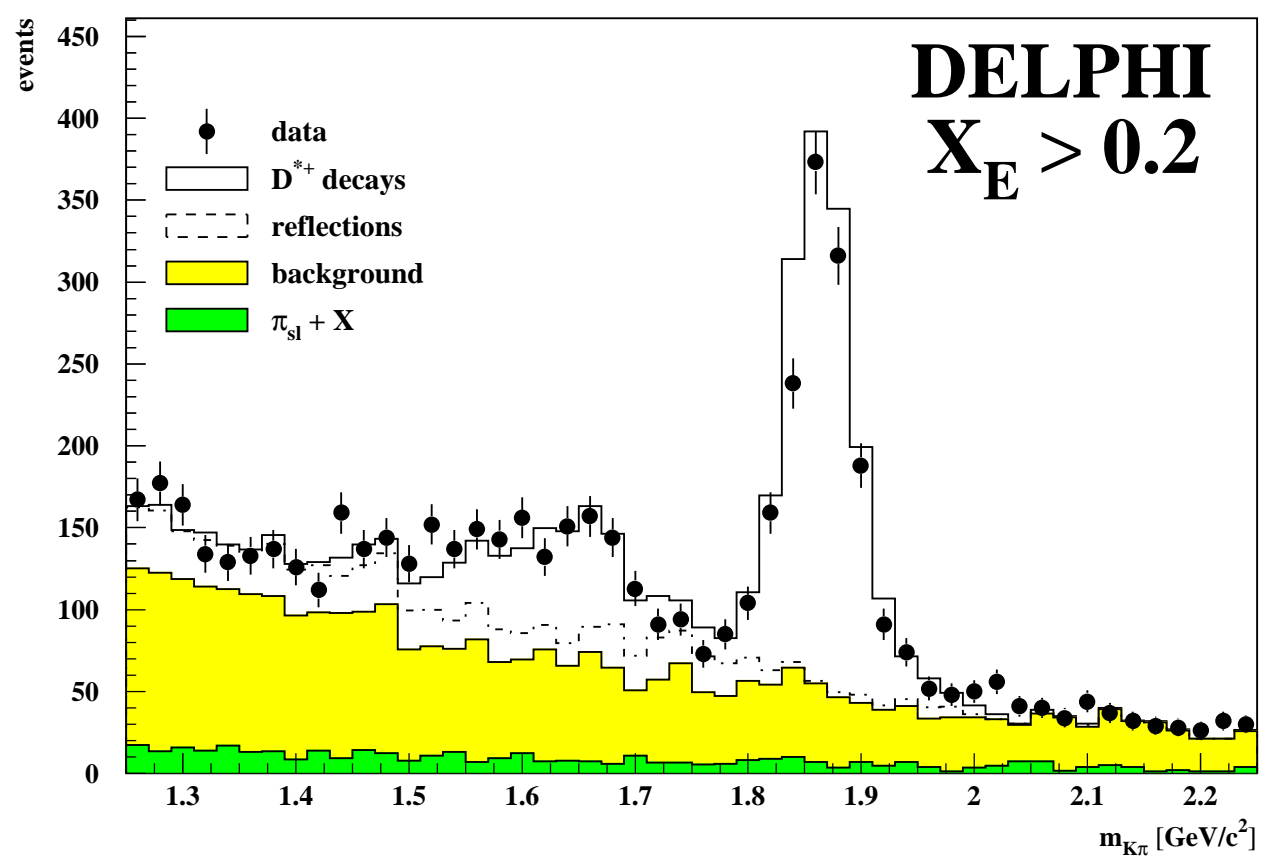

Figure 18: Invariant mass spectrum of $\mathrm{D}^{0}$ candidates reconstructed in the DELPHI detector. The second broader peak visible around $1.6 \mathrm{GeV}$ is due to the decay $\mathrm{D}^{0} \rightarrow \mathrm{K} \pi \pi^{0}$, with the $\pi^{0}$ not reconstructed.

with the definitions of the $f_{i}, \chi_{\mathrm{D}^{*+}}$ and $A_{\mathrm{FB}}^{\mathrm{bkgd}}$ equivalent to those of equation $(29)$. The $\mathrm{B}-\overline{\mathrm{B}}$ mixing parameter, $\chi_{\mathrm{D}^{*+}}$, is that appropriate to the sample of $\mathrm{D}^{*+}$ mesons, which include mostly neutral B mesons and a small fraction of charged B mesons. "Wrongly" charged $\mathrm{D}^{*-}$ mesons can be produced in b-decays via the suppressed production of a $\bar{c}$ quark in the process $b \rightarrow c \bar{c} s$. This component is estimated to be less than 1\% from Monte Carlo studies and theoretical considerations [44]. To ensure that $A_{\mathrm{FB}}^{\mathrm{bkg}}=0$, partially reconstructed $\mathrm{D}$ mesons, present in the tagged samples at a significant level, must be counted as part of the signal. They are handled in different ways by the different collaborations, and are most often corrected for using Monte Carlo models. OPAL use a special estimator constructed from the data.

A distribution of $Q \cos \theta$ of identified candidates, corrected for acceptance, is shown in figure 19. The sensitivity of these tags is largest for charm, and it can be improved by combination with the asymmetries obtained from the lepton measurements discussed above, taking the correlations between the measurements into account. As an example, table 6 shows the correlation matrix between the OPAL asymmetry measurement using leptons and that using $\mathrm{D}^{*+}$ mesons [43]. Note that the largest correlations are between the $\mathrm{b}$ and $\mathrm{c}$ asymmetries measured within each analysis, but that these correlations have opposite signs in the two cases. Averaging the results therefore gives both an improved accuracy and a reduced correlation between the measured $b$ and $c$ asymmetries. A full combination of all the asymmetry results is discussed in section 7 .

The results from the different asymmetry measurements with reconstructed particles are listed in table 7 . These measurements are dominated by statistical errors, and are likely to remain so for some time in future. 


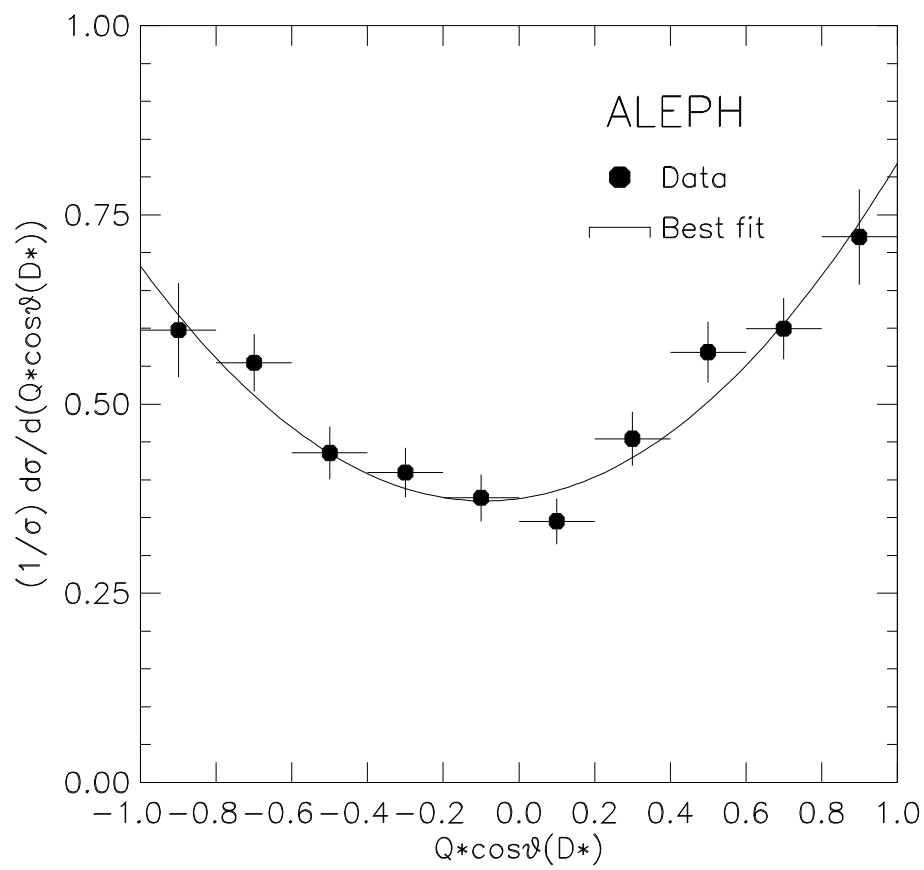

Figure 19: Distribution of $Q \cos \theta_{\text {thrust }}$ for $\mathrm{D}^{*+}$ candidates after background subtraction and correction for the acceptance as observed with the ALEPH detector. The solid curve shows the expected shape given the experimental results for the asymmetry.

\begin{tabular}{|c|cccc|}
\hline & $A_{\mathrm{FB}}^{\mathrm{c}}(l)$ & $A_{\mathrm{FB}}^{\mathrm{b}}(l)$ & $A_{\mathrm{FB}}^{\mathrm{c}}\left(\mathrm{D}^{*+}\right)$ & $A_{\mathrm{FB}}^{\mathrm{b}}\left(\mathrm{D}^{*+}\right)$ \\
\hline \hline$A_{\mathrm{FB}}^{\mathrm{c}}(l)$ & 1.00 & 0.26 & 0.01 & 0.06 \\
$A_{\mathrm{FB}}^{\mathrm{b}}(l)$ & & 1.00 & 0.01 & 0.05 \\
$A_{\mathrm{FB}}^{\mathrm{c}}\left(\mathrm{D}^{*+}\right)$ & & & 1.00 & -0.78 \\
$A_{\mathrm{FB}}^{\mathrm{b}}\left(\mathrm{D}^{*+}\right)$ & & & & 1.00 \\
\hline
\end{tabular}

Table 6: Correlation coefficients for the combination of the lepton and $\mathrm{D}^{*+}$ asymmetry measurement including all statistical and systematic errors, as published by the OPAL collaboration [43].

\begin{tabular}{|c|c|c|c|}
\hline Experiment & $A_{\mathrm{FB}}^{\mathrm{c}}{ }^{\text {const }}$ & $A_{\mathrm{FB}}^{\mathrm{c}}$ & $A_{\mathrm{FB}}^{\mathrm{b}}$ \\
\hline \hline ALEPH [25] & $\mathbf{0 . 0 6 8} \pm \mathbf{0 . 0 4 2} \pm \mathbf{0 . 0 0 9}$ & & \\
DELPHI [42] & $\mathbf{0 . 0 6 9} \pm \mathbf{0 . 0 2 7} \pm \mathbf{0 . 0 1 1}$ & $\mathbf{0 . 0 8 1} \pm \mathbf{0 . 0 2 9} \pm \mathbf{0 . 0 1 2}$ & $\mathbf{0 . 0 4 6} \pm \mathbf{0 . 0 5 9} \pm \mathbf{0 . 0 2 6}$ \\
OPAL [43] & $\mathbf{0 . 0 9 2 \pm 0 . 0 2 4 \pm 0 . 0 1 1}$ & $\mathbf{0 . 1 1 0 \pm 0 . 0 3 7 \pm 0 . 0 1 2}$ & $\mathbf{0 . 0 4 2 \pm 0 . 0 7 9 \pm 0 . 0 2 5}$ \\
\hline
\end{tabular}

Table 7: Charm and b forward-backward asymmetries as measured using $\mathrm{D}^{*+}$ mesons. The quantity $A_{\mathrm{FB}}^{\mathrm{c}}{ }^{\text {const }}$ is the $\mathrm{c}$ asymmetry obtained after constraining the $\mathrm{b}$ asymmetry to its value measured using high momentum leptons. The other two columns show the results from a simultaneous fit of $A_{\mathrm{FB}}^{\mathrm{c}}$ and $A_{\mathrm{FB}}^{\mathrm{b}}$. Corrections needed to convert these values into pole asymmetries have not been applied. The first error quoted is always the statistical error, the second one the systematic. 


\subsection{Asymmetry Measurements with Lifetime Tagged Events}

As discussed in section 3.5 above, the primary quark charge in a lifetime-tagged $\mathrm{b}$ hemisphere can be estimated using the jet-charge technique. ALEPH [33] and DELPHI [14] have published analyses using such an approach. Precise silicon vertex detectors only cover the central part of the acceptance for data analysed so far, to approximately $|\cos \theta|=0.75-0.8$. The lifetime tagging performance is thus significantly degraded outside this region, so the thrust axis is required to lie within this acceptance. ALEPH use a cut at $\left|\cos \theta_{\text {thrust }}\right|=0.8$, DELPHI one at $\left|\cos \theta_{\text {thrust }}\right|=0.7$. Both analyses measure the $b$ quark tagging efficiency of the lifetime tag from the data using the double-tagging approach described in section 4. Some details of the ALEPH and DELPHI approaches are quite different - ALEPH use the statistical separation power of the hemisphere charge measure, DELPHI use an event-by-event charge tag and exploit leptons to measure its reliability.

ALEPH measure the forward-backward asymmetry using the relationship (section 3.5 and equation (15)):

$$
<Q_{F B}>\equiv<Q_{F}-Q_{B}>\simeq \delta^{\mathrm{b}} A_{\mathrm{FB}}^{\mathrm{b}}
$$

where $\delta^{\mathrm{b}}$ is the value of $\delta$ for b quarks. No binning in $\cos \theta_{\text {thrust }}$ is used, and $\delta_{b}$ is determined from the data, with only small corrections from Monte Carlo. The residual lighter quark background in the sample also gives a contribution to the overall asymmetry: ALEPH choose to take the standard model predictions for the dependence of all five quark flavour asymmetries on $\sin ^{2} \theta_{\mathrm{W}}^{\text {eff }}$, and they then fit for the single parameter $\sin ^{2} \theta_{\mathrm{W}}^{\text {eff }}$. This is then converted back, using the standard model relationship, into the value of $A_{\mathrm{FB}}^{\mathrm{b}}$ at the $\mathrm{Z}^{0}$ peak.

DELPHI measure the forward-backward asymmetry using a chi-squared fit to the distribution of $\cos \theta_{\text {thrust }}$ of the tagged sample, taking the charge assignment according to which hemisphere has the lower measured charge. Corrections are applied for the lighter quark background, assuming that $A_{\mathrm{FB}}^{\mathrm{b}}=A_{\mathrm{FB}}^{\mathrm{s}}=A_{\mathrm{FB}}^{\mathrm{d}}$ and $A_{\mathrm{FB}}^{\mathrm{c}}=A_{\mathrm{FB}}^{\mathrm{u}}$ as predicted approximately by the standard model, and taking $A_{\mathrm{FB}}^{\mathrm{c}}=\lambda A_{\mathrm{FB}}^{\mathrm{b}}$, where the scale factor $\lambda$ is taken to have its standard model value.

The final results obtained in the two analyses are shown in table 8 . It can be seen from

\begin{tabular}{|l|c|}
\hline & $A_{\mathrm{FB}}^{\mathrm{b}}$ \\
\hline ALEPH [33] & $\mathbf{0 . 0 9 9 2 \pm \mathbf { 0 . 0 0 8 4 } \pm \mathbf { 0 . 0 0 4 6 }}$ \\
DELPHI [14] & $\mathbf{0 . 1 1 5} \pm \mathbf{0 . 0 1 7} \pm \mathbf{0 . 0 1 0}$ \\
\hline
\end{tabular}

Table 8: Current published results for $A_{\mathrm{FB}}^{\mathrm{b}}$ using lifetime tags and hemisphere charge measures. Corrections needed to convert these values into pole asymmetries have not been applied. The first error shown in each case is statistical, the second systematic.

the table that the measurements are statistically limited. The dominant sources of systematic error in both ALEPH and DELPHI analyses come from knowledge of how well the charge identification performs, and the purity of the $b$ tag. Both of these are determined largely from the data, and so they will shrink as the data volume increases, giving promise that this type of analysis will continue to be statistically limited. 


\subsection{Corrections and Theoretical Uncertainties}

The forward-backward asymmetry measurements described almost all assume that the differential cross-section can be written (equation (6)):

$$
\frac{\mathrm{d} \sigma}{\mathrm{d} \cos \theta} \propto 1+\cos ^{2} \theta+\frac{8}{3} A_{\mathrm{FB}}^{\mathrm{f}} \cos \theta
$$

and that the quark direction is well approximated by the thrust axis direction. Conversion of these measured asymmetries, $A_{\mathrm{FB}}^{\mathrm{b}}$ and $A_{\mathrm{FB}}^{\mathrm{c}}$, to the pole asymmetries, $A_{\mathrm{FB}}^{\mathrm{b}, 0}$ and $A_{\mathrm{FB}}^{\mathrm{c}, 0}$, therefore requires some small corrections to account for these assumptions. In addition, radiation of photons by the incoming electron and positron is not included in the definition of the pole asymmetries, but is present in the data. These corrections needed to convert the measured asymmetries to the pole asymmetries are listed in table 9 .

\begin{tabular}{|l|l|l|}
\hline Source & Shift in $A_{\mathrm{FB}}^{\mathrm{b}}$ & Shift in $A_{\mathrm{FB}}^{\mathrm{c}}$ \\
\hline QED corrections & $+\mathbf{0 . 0 0 4 1}$ & $+\mathbf{0 . 0 1 0 4}$ \\
QCD corrections & $+\mathbf{0 . 0 0 2 7} \pm \mathbf{0 . 0 0 1 0}$ & $+\mathbf{0 . 0 0 2 2} \pm \mathbf{0 . 0 0 0 5}$ \\
Photon exchange & $-\mathbf{0 . 0 0 0 3}$ & $-\mathbf{0 . 0 0 0 8}$ \\
Centre-of-mass energy & $-\mathbf{0 . 0 0 1 3}$ & $-\mathbf{0 . 0 0 3 4}$ \\
\hline Overall & $+\mathbf{0 . 0 0 5 2 \pm \mathbf { 0 . 0 0 1 0 }}$ & $+\mathbf{0 . 0 0 8 4} \pm \mathbf{0 . 0 0 0 5}$ \\
\hline
\end{tabular}

Table 9: Corrections needing to be applied to the measured $\mathrm{b}$ and $\mathrm{c}$ quark asymmetries [2,3,45]. The shifts shown are added to the measured asymmetries to obtain the corrected values. The uncertainty on the QCD correction is much larger than those on the other corrections.

QED corrections include primarily the effects of initial state radiation. This correction is particularly important because the forward-backward asymmetry varies quite strongly with centre-of-mass energy. The QCD correction for using the thrust axis to approximate the quark direction has been calculated for massless quarks, and a modification of the correction for the massive quark case has been estimated by Lampe et al. [45]. In addition, $\mathrm{e}^{+} \mathrm{e}^{-}$annihilation through the photon instead of the $\mathrm{Z}^{0}$ also affects the asymmetry slightly, and a further small correction needs to be applied to correct from the centre-of-mass energy at which LEP has operated to the nearby $\mathrm{Z}^{0}$ mass.

\subsection{Combined Results}

The results of the on-peak asymmetry measurements using the different techniques are shown in figures 20 and 21. The averages shown are derived taking into account the full correlations between measurements, discussed in the following section. The averages have been derived for a centre-of-mass energy, $\sqrt{s}$, of $91.26 \mathrm{GeV}$. For the b asymmetry, lepton and jet charge measurements contribute approximately equally. Measurements using reconstructed particles are not shown in figure 20 because they do not contribute significantly to the $b$ asymmetry measurement. For the $\mathrm{c}$ asymmetry, lepton and reconstructed particle measurements contribute similarly.

Measurements of the $b$ and $c$ asymmetry at energies at the sides of the peak of the $Z^{0}$ resonance are listed in table 10 . The $\mathrm{b}$ asymmetry has been measured using leptons by a 


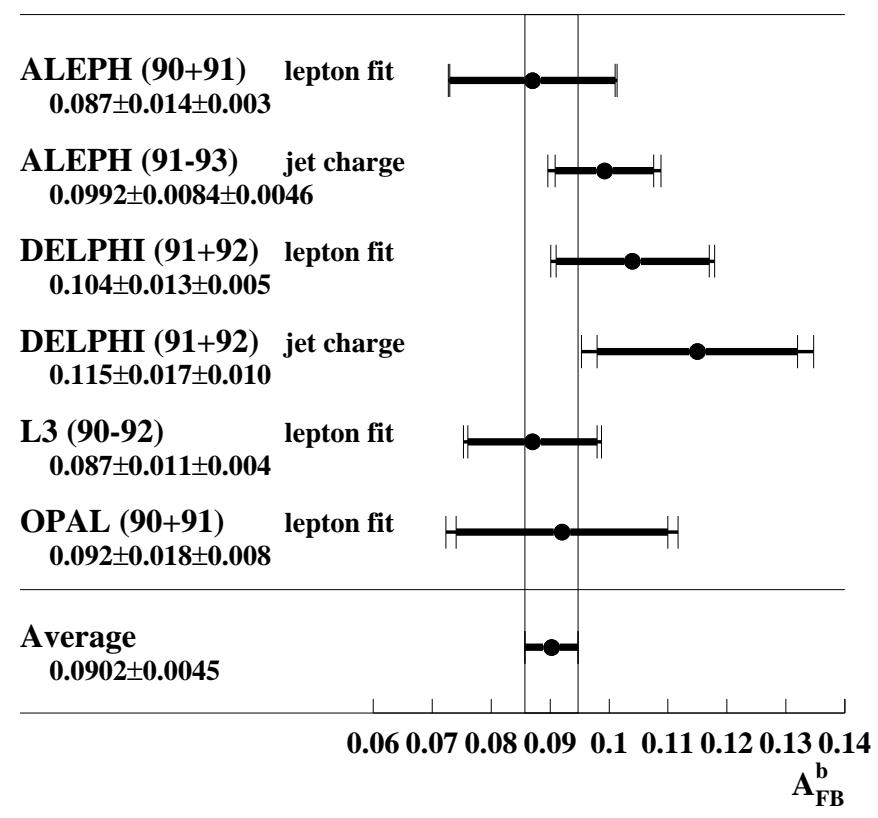

Figure 20: Comparison of current measurements of the $b$ forward-backward asymmetry from LEP [14, $17,19,33,40]$. The average shown is obtained from the fit to all heavy quark electroweak quantities, as described in the text. Measurements of the $b$ asymmetry with $\mathrm{D}^{*+}$ tags are not shown here as they do not contribute significantly to the average. They are included in the overall fit.

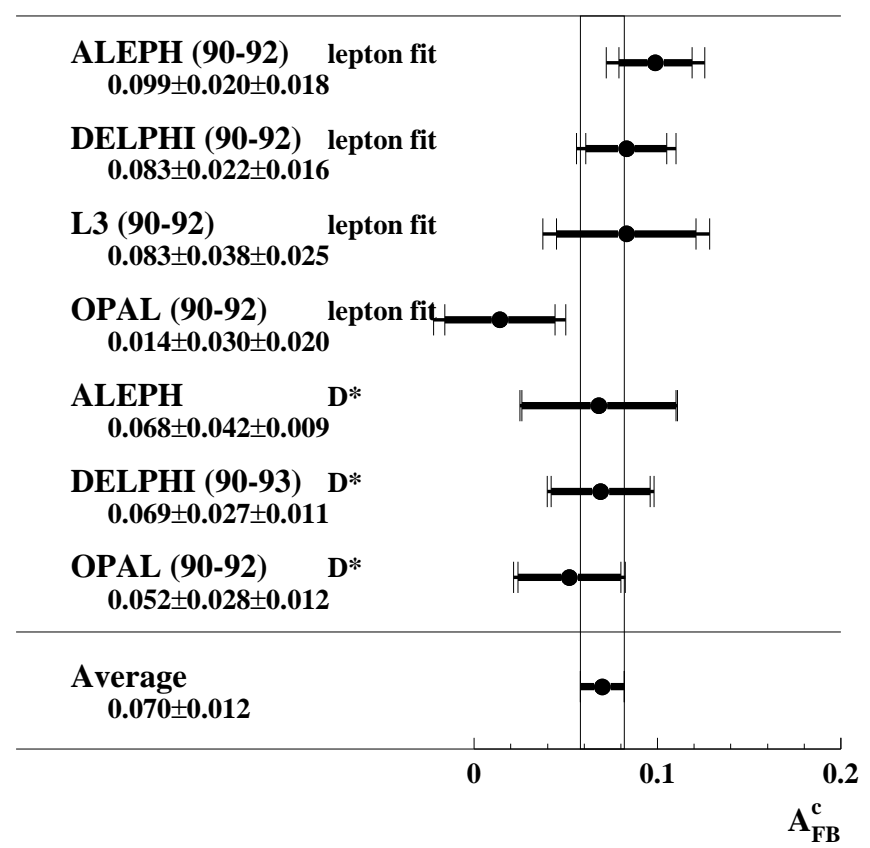

Figure 21: Compilation of published measurements of the charm asymmetry measured at LEP [14, $17,19,25,27,31,40]$. The individual values are quoted as they appear in the original publications. For the calculation of the average, correlations between measurements have been taken into account as discussed in the text. 


\begin{tabular}{|c|c|c|}
\hline & $\sqrt{s}(\mathrm{GeV})$ & $A_{\mathrm{FB}}^{\mathrm{b}}$ \\
\hline \hline ALEPH [19] & -3 & $\mathbf{0 . 0 3 8} \pm \mathbf{0 . 0 6 7}$ \\
ALEPH [19] & -2 & $-\mathbf{0 . 0 1 7} \pm \mathbf{0 . 0 7 6}$ \\
ALEPH [19] & -1 & $\mathbf{0 . 0 4 5} \pm \mathbf{0 . 0 6 0}$ \\
ALEPH [19] & +1 & $\mathbf{0 . 0 7 0} \pm \mathbf{0 . 0 5 5}$ \\
ALEPH [19] & +2 & $\mathbf{0 . 1 2 1} \pm \mathbf{0 . 0 7 0}$ \\
ALEPH [19] & +3 & $\mathbf{0 . 1 4 5} \pm \mathbf{0 . 0 8 2}$ \\
L3 [41] & 89.67 & $\mathbf{0 . 0 2 5} \pm \mathbf{0 . 0 5 1}$ \\
L3 [41] & 92.81 & $\mathbf{0 . 0 6 2} \pm \mathbf{0 . 0 4 3}$ \\
OPAL [40] & 89.66 & $\mathbf{0 . 0 7 1} \pm \mathbf{0 . 0 5 4}$ \\
OPAL [40] & 92.75 & $\mathbf{0 . 1 3 1} \pm \mathbf{0 . 0 4 9}$ \\
\hline \hline & $\sqrt{s}$ & $A_{\mathrm{FB}}^{\mathrm{c}}$ \\
\hline \hline OPAL [43] & 89.75 & $-\mathbf{0 . 1 4} \pm \mathbf{0 . 1 4}$ \\
OPAL [43] & 92.64 & $\mathbf{0 . 1 8} \pm \mathbf{0 . 1 2}$ \\
\hline
\end{tabular}

Table 10: Published results for $A_{\mathrm{FB}}^{\mathrm{b}}$ and $A_{\mathrm{FB}}^{\mathrm{c}}$ for centre-of-mass energies displaced from the $\mathrm{Z}^{0}$ peak. All the $A_{\mathrm{FB}}^{\mathrm{b}}$ measurements use lepton tagging, the $A_{\mathrm{FB}}^{\mathrm{c}}$ measurements instead use $\mathrm{D}^{*+}$ tags. The errors shown include both statistical and systematic contributions. Statistical errors dominate the results. Energies denoted " $\pm 1, \pm 2, \pm 3$ " indicate shifts from the on-peak centre-of-mass energy of $91.2 \mathrm{GeV}$.

number of experiments. Only OPAL has published a measurement of the c asymmetry at the off-peak energy points. The errors are much larger than on-peak because the large majority of the data collected has been taken at the peak.

\section{Comparison with Standard Model Predictions}

All analyses presented above, while usually attempting to measure a limited set of quantities, are sensitive at some level to several of the electroweak observables. For example, asymmetry measurements are sensitive to the partial width into pairs of heavy quarks. In addition many of the systematic errors are common between different experiments, or between different analyses performed by the same experiment. In the latter case statistical correlations have to be considered as well.

It is important to treat these correlations in order to obtain reliable and consistent averages of all the results. The LEP electroweak working group [3] has defined a procedure for averaging the different results, based on a chi-squared minimisation, taking these common and correlated errors properly into account. This averaging procedure has been adopted in deriving the averages quoted in this and preceding sections.

Further complications arise since many measurements share systematic errors from such sources as the $b$ semileptonic branching ratio, and $\mathrm{B}-\overline{\mathrm{B}}$ mixing. Since some of the lepton fit measurements themselves measure these sources, they are included in the averaging fit. Individual results quoted by experiments generally use slightly different ranges of parameters in evaluating systematic errors. However, the LEP experiments have recently agreed upon a standard set of systematic parameter variations [3], which simplifies combination of results from the different experiments. These produce only small shifts in the results quoted in the previous 
sections, and small changes in systematic errors. These standard parameter variations have been adopted for the averages quoted here as well.

Applying this averaging procedure to all the results described above yields, for a centre-ofmass energy of $91.26 \mathrm{GeV}$ :

$$
\begin{aligned}
\Gamma_{\mathrm{b} \overline{\mathrm{b}}} / \Gamma_{\text {had }} & =0.2207 \pm 0.0022 \\
\Gamma_{\mathrm{c} \overline{\mathbf{c}}} / \Gamma_{\mathrm{had}} & =0.153 \pm \mathbf{0 . 0 1 1} \\
A_{\mathrm{FB}}^{\mathrm{b}} & =0.0902 \pm 0.0045 \\
A_{\mathrm{FB}}^{\mathrm{c}} & =0.070 \pm 0.012
\end{aligned}
$$

and also gives values of $B(\mathrm{~b} \rightarrow \ell)=(11.0 \pm 0.4) \%, B\left(\mathrm{~b} \rightarrow \mathrm{c} \rightarrow \ell^{+}\right)=(8.2 \pm 0.5) \%$, and $\chi=0.113 \pm 0.009$. Exactly these seven parameters are fitted, and the errors include statistical and systematic effects. Note that only the on-peak asymmetry measurements are included. The correlation matrix of the combined measurements is shown in table 11 . The biggest correlation between the four electroweak parameters is that between $\Gamma_{\mathrm{b}} \bar{b} / \Gamma_{\text {had }}$ and $\Gamma_{\mathbf{c} \bar{c}} / \Gamma_{\text {had }}$, of $-39 \%$. This is a reflection of the fact that all the tagging methods discussed tag both $b$ and $c$ quarks with higher probability than lighter quarks.

\begin{tabular}{|l|c|c|c|c|c|c|c|}
\hline & $\Gamma_{\mathrm{b} \overline{\mathrm{b}}} / \Gamma_{\mathrm{had}}$ & $\Gamma_{\mathrm{c} \overline{\mathrm{c}}} / \Gamma_{\mathrm{had}}$ & $A_{\mathrm{FB}}^{\mathrm{b}}$ & $A_{\mathrm{FB}}^{\mathrm{c}}$ & $\mathrm{B}(\mathrm{b} \rightarrow \ell)$ & $\mathrm{B}\left(\mathrm{b} \rightarrow \mathrm{c} \rightarrow \ell^{+}\right)$ & $\chi$ \\
\hline$\Gamma_{\mathrm{b} \overline{\mathrm{b}}} / \Gamma_{\mathrm{had}}$ & 1 & -0.39 & -0.04 & 0.09 & -0.20 & -0.01 & -0.02 \\
$\Gamma_{\mathrm{c} \overline{\mathrm{c}}} / \Gamma_{\mathrm{had}}$ & & 1 & 0.14 & -0.12 & 0.21 & 0.01 & -0.01 \\
$A_{\mathrm{FB}}^{\mathrm{b}}$ & & & 1 & 0.07 & 0.07 & -0.05 & 0.19 \\
$A_{\mathrm{FB}}^{\mathrm{c}}$ & & & & 1 & 0.09 & -0.15 & 0.05 \\
$\mathrm{~B}(\mathrm{~b} \rightarrow \ell)$ & & & & & 1 & 0.05 & 0.35 \\
$\mathrm{~B}(\mathrm{~b} \rightarrow \mathrm{c} \rightarrow \ell)$ & & & & & & 1 & -0.39 \\
$\chi$ & & & & & & & 1 \\
\hline
\end{tabular}

Table 11: Correlation coefficients between averaged heavy quark electroweak results from the overall fit.

The results for the partial widths, $\Gamma_{\mathrm{b}} \overline{\mathrm{b}} / \Gamma_{\text {had }}$ and $\Gamma_{\mathbf{c} \bar{c}} / \Gamma_{\mathrm{had}}$, from this consistent fit differ slightly from the averages quoted in sections 4 and 5 , because in the earlier averages fixed (standard model) values were used for the other partial widths. The averages from the full fit are therefore of most interest in testing if the full set of LEP heavy quark electroweak measurements are consistent with the standard model. They provide an unbiased measurement of these quantities, without assuming a particular model.

The combined values of $\Gamma_{\mathrm{b}} / \Gamma_{\text {had }}$ and $\Gamma_{\mathbf{c} \overline{\mathrm{c}}} / \Gamma_{\text {had }}$ are shown in figure 22 , and compared with the standard model prediction of ZFITTER [2]. Agreement is at the level of approximately two standard deviations, with the data tending to favour a higher $\Gamma_{\mathrm{b}} / \Gamma_{\text {had }}$ value and lower $\Gamma_{\mathbf{c} \overline{\mathbf{c}}} / \Gamma_{\text {had }}$ value than predicted.

It is also interesting to consider the implications of the results within the framework of the standard model. In particular, as discussed in section 2 , a precise measurement of $\Gamma_{\mathrm{b}} / \Gamma_{\text {had }}$ is sensitive to the top quark mass, but $\Gamma_{\mathbf{c} \bar{c}} / \Gamma_{\text {had }}$ is relatively insensitive. The experimental measurements of $\Gamma_{\mathrm{b}} / \Gamma_{\text {had }}$ are also much more precise than those of $\Gamma_{\mathbf{c} \bar{c}} / \Gamma_{\text {had }}$. In the standard model framework, it is therefore sensible to fix $\Gamma_{\mathbf{c} \overline{\mathbf{c}}} / \Gamma_{\text {had }}$ to its standard model value before deriving the $\Gamma_{\mathrm{bb}} / \Gamma_{\text {had }}$ average, as was done in section 4 . That average result is compared in 


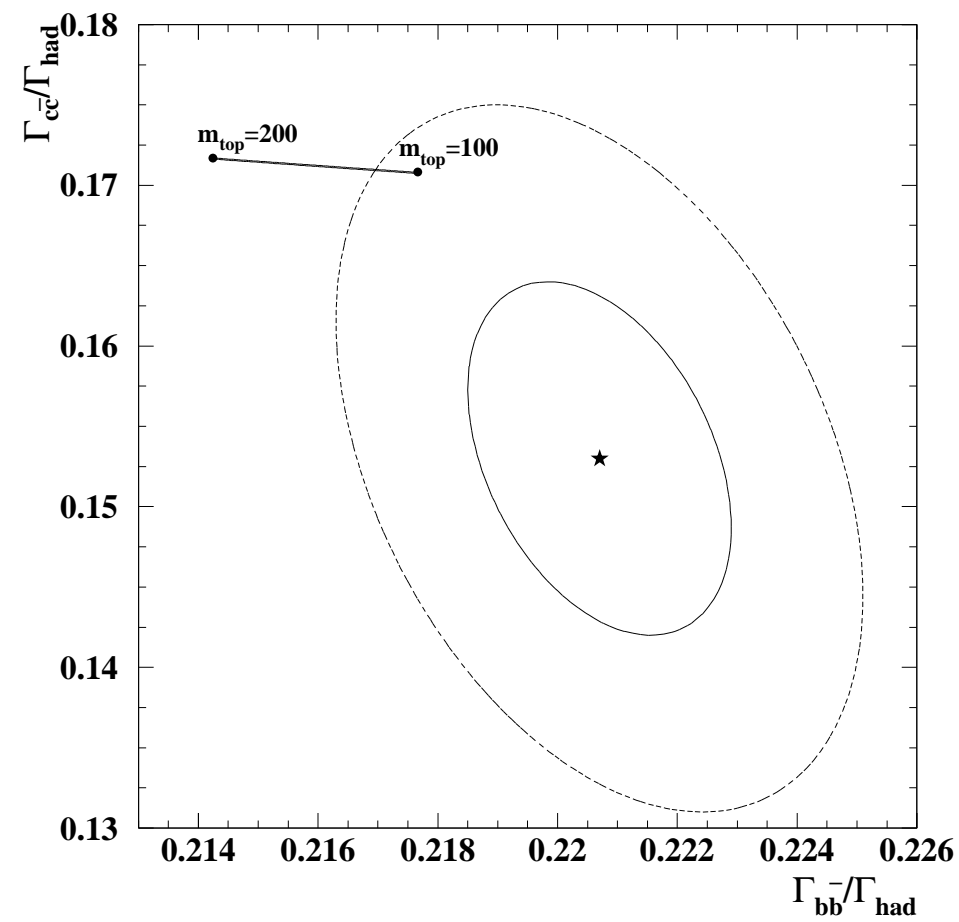

Figure 22: Comparison of the averaged measured $\Gamma_{\mathrm{b}} / \Gamma_{\text {had }}$ and $\Gamma_{\mathrm{c} \bar{c}} / \Gamma_{\text {had }}$ values with the standard model prediction [2]. The central value is shown as a small star, the contours correspond to one and two standard deviations, and so have $39 \%$ and $86 \%$ probability content, respectively. The line to the top left shows the standard model prediction, varying the top mass between 100 and $200 \mathrm{GeV}$. The thickness of the line shows the very small effect of varying the Higgs mass between 60 and $1000 \mathrm{GeV}$.

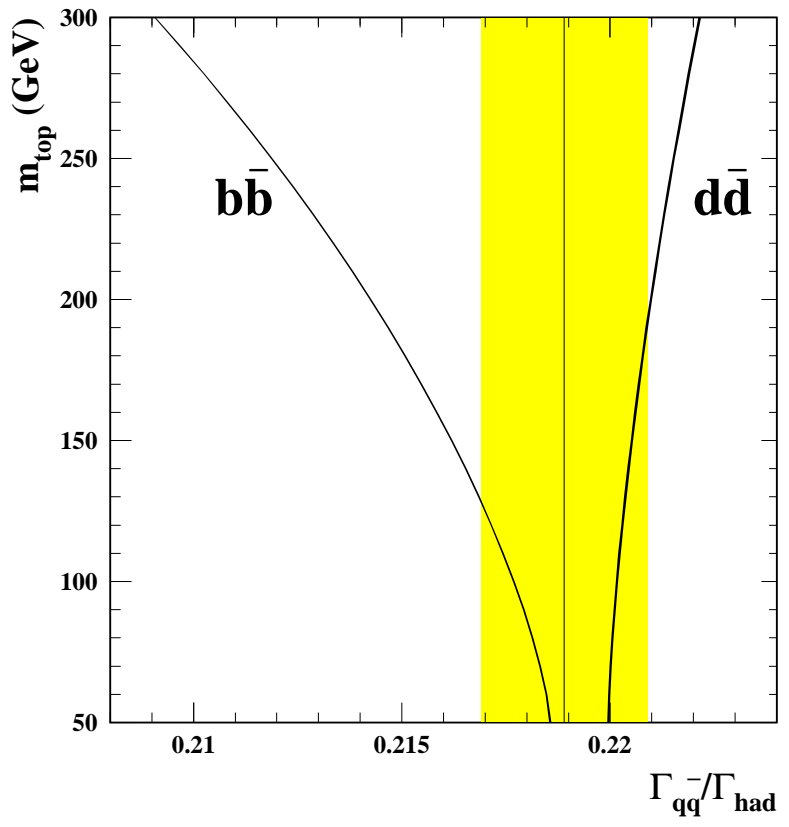

Figure 23: Comparison of average $\Gamma_{\mathrm{b}} / \Gamma_{\mathrm{had}}\left(\Gamma_{\mathrm{c} \bar{c}} / \Gamma_{\text {had }}=0.171\right)$ with the standard model prediction as a function of top mass [2]. 
figure 23 with the standard model prediction as a function of the top mass. In the standard model framework, the result favours a low top mass value, but is consistent at the one standard deviation level with top masses up to $129 \mathrm{GeV}$. The result can be interpreted as a constraint on the top mass, that it is less that $183 \mathrm{GeV}$ at the $95 \%$ confidence level. As was noted in section 2, this particular top mass constraint has very little dependence on the unknown mass of the Higgs boson, in contrast to most other top mass limits derived via radiative corrections to electroweak measurements at the $\mathrm{Z}^{0}$.

The average on-peak asymmetries can be converted into pole asymmetries using the corrections in table 9:

$$
\begin{aligned}
& A_{\mathrm{FB}}^{\mathrm{b}, 0}=0.0954 \pm 0.0046 \\
& A_{\mathrm{FB}}^{\mathrm{c}, 0}=0.078 \pm 0.012
\end{aligned}
$$

These values can be interpreted as measurements of the electroweak mixing angle $\sin ^{2} \theta_{\mathrm{W}}^{\text {eff }}$ :

$$
\sin ^{2} \theta_{\mathrm{W}}^{\mathrm{eff}}=0.2328 \pm 0.0008
$$

from $A_{\mathrm{FB}}^{\mathrm{b}}$, and

$$
\sin ^{2} \theta_{\mathrm{W}}^{\mathrm{eff}}=0.2305 \pm 0.0028
$$

from $A_{\mathrm{FB}}^{\mathrm{c}}$. The $\mathrm{b}$ forward-backward asymmetry gives a substantially more precise measurement of $\sin ^{2} \theta_{\mathrm{W}}^{\mathrm{eff}}$ than $A_{\mathrm{FB}}^{\mathrm{c}}$, as anticipated in section 2 . The error on the $\sin ^{2} \theta_{\mathrm{W}}^{\mathrm{eff}}$ value derived from $A_{\mathrm{FB}}^{\mathrm{b}}$ in fact compares very favourably with that possible using other observables at LEP.

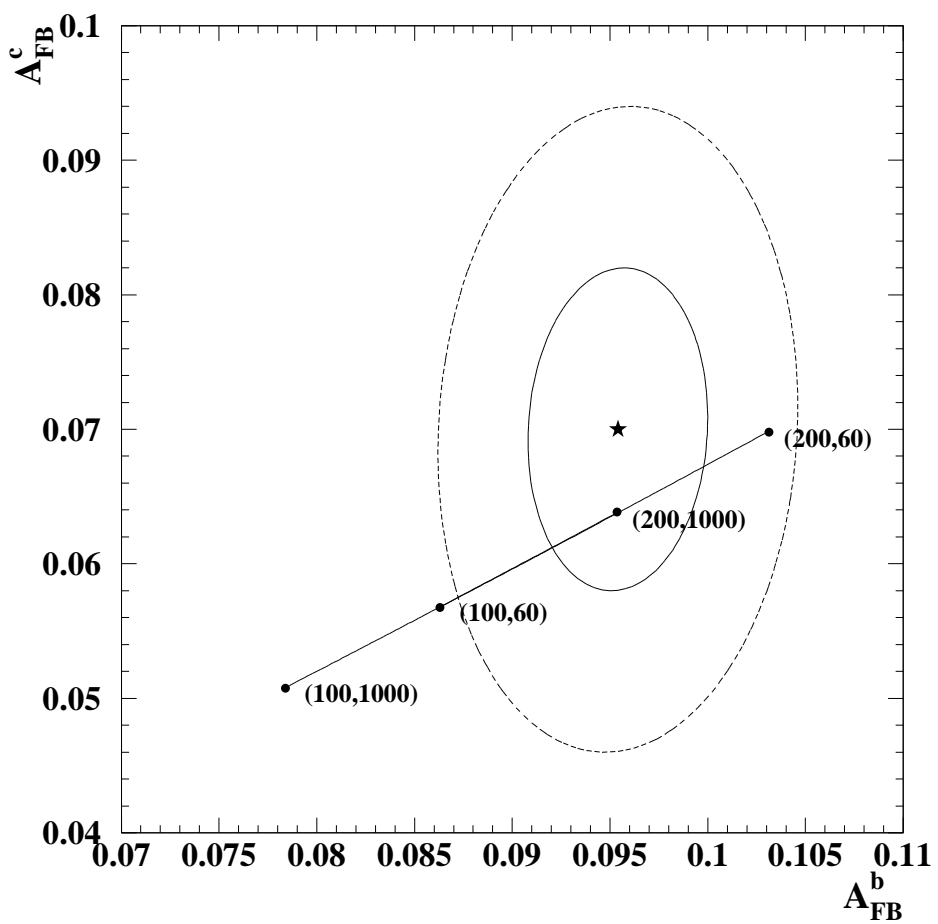

Figure 24: Comparison of the averaged measured $A_{\mathrm{FB}}^{\mathrm{b}}$ and $A_{\mathrm{FB}}^{\mathrm{c}}$ values with the standard model prediction [2]. The central value is shown as a small star, the contours correspond to one and two standard deviations, and so have $39 \%$ and $86 \%$ probability content, respectively. The line shown corresponds to the standard model prediction, for the values (in $\mathrm{GeV})$ of $\left(m_{\mathrm{top}}, m_{\mathrm{Higgs}}\right)$ shown. 

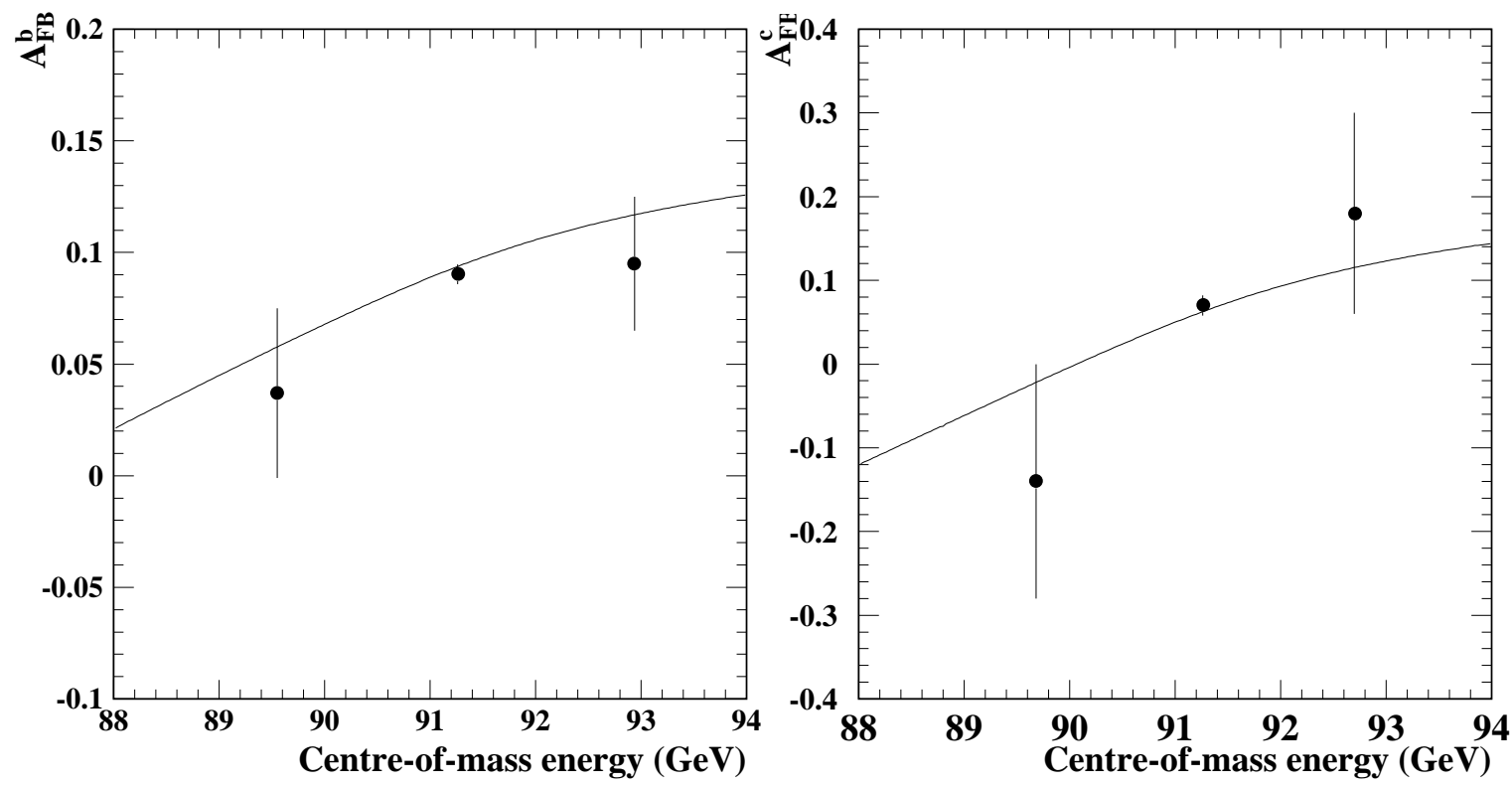

Figure 25: Averaged measurements of the $\mathrm{b}$ and $\mathrm{c}$ asymmetries at energies on and around the pole of the $\mathrm{Z}^{0}$ resonance. The curve shown is the prediction of the standard model for a Higgs mass of $300 \mathrm{GeV}$ and a top mass of $175 \mathrm{GeV}$.

The measured asymmetry values are compared in figure 24 with the standard model prediction of ZFITTER [2]. Agreement is good.

The averaged values measured for the $b$ and $c$ asymmetries above and below the $Z^{0}$ peak are shown in figure 25 together with the average on-peak asymmetry. The averages were performed using the same chi-squared minimisation technique as used for the main combined average, assuming that the partial widths, semileptonic branching ratios, and $\chi$ mixing parameter can be taken from the on-peak measurements. The curves show the standard model predictions, from ZFITTER [2].

Recently the SLD collaboration, working with polarised electron beams at the SLC linear collider, have reported measurements of the $b$ and $c$ quark forward-backward left-right asymmetries [46], which probe directly the coupling constants $\mathcal{A}_{\mathrm{b}}$ and $\mathcal{A}_{\mathrm{c}}$. They find:

$$
\begin{aligned}
& \mathcal{A}_{\mathrm{b}}=0.89 \pm 0.09 \pm 0.06 \\
& \mathcal{A}_{\mathrm{c}}=0.37 \pm 0.23 \pm 0.21,
\end{aligned}
$$

where the first error shown is statistical, the second systematic. The results are in agreement with the predictions of the standard model of $\mathcal{A}_{\mathrm{b}} \approx 0.94, \mathcal{A}_{\mathbf{c}} \approx 0.67$ (see section 2 ), and with the LEP measurements presented here.

\section{$8 \quad$ Light Quark Properties}

An interesting extension of the measurements discussed in this review is the determination of the partial widths and the asymmetries in the light quark sector. Such measurements also provide tests of the universality of the couplings in the standard model. 
Undifferentiated average "up-like" and "down-like" partial widths have been determined using events with a final state photon [47] together with the precisely measured total $\mathrm{Z}^{0}$ hadronic decay width, $\Gamma_{\text {had }}$, and are measured at the 10\%-20\% level. Similarily the asymmetries of all hadrons have been measured by all four LEP experiments, without distinguishing between primary event flavours, using momentum weighted event charge techniques. They are used to derive values for $\sin ^{2} \theta_{\mathrm{W}}^{\mathrm{eff}}[48]$.

Recently techniques for tagging light flavour events using high momentum stable particles have been developed $[49,50]$. A first measurement of the asymmetry of strange quarks at LEP has been presented by the DELPHI collaboration [50]. Fast charged kaons, and $\Lambda^{0}$ baryons decaying into $\mathrm{p} \pi^{-}$, are used as tags for strange quarks. Unlike the analyses using heavy quarks, a significant fraction of these particles are produced in the fragmentation process or from decays of $b$ and $c$ quarks. A measurement of the apparent asymmetry of the kaon is therefore not trivially related to the corresponding strange quark asymmetry. Rather involved hadronisation corrections have to be applied, which are currently based on Monte Carlo studies. Using in addition only the assumption that the unmeasured $\mathrm{u}$ and $\mathrm{d}$ quark asymmetries are respectively equal to the $c$ and $b$ quark asymmetries leads to

$$
A_{\mathrm{FB}}^{\mathrm{s}}=0.131 \pm 0.025 \pm 0.054
$$

where the first error is statistical, the second systematic. The systematic error is dominated by modelling errors in the Monte Carlo, in particular of the charm fragmentation and the probability of producing $s \bar{s}$ pairs in the hadronisation process.

The result is consistent with the b asymmetry measurements presented above, illustrating the flavour independence of the couplings in the standard model, at the current level of precision.

\section{$9 \quad$ Future Prospects}

\begin{tabular}{|l|c|}
\hline Year & Million hadronic $\mathrm{Z}^{0}$ decays \\
\hline $1989(\operatorname{sca})$ & 0.03 \\
$1990($ scan $)$ & 0.15 \\
$1991($ scan $)$ & 0.3 \\
1992 & 0.7 \\
$1993($ scan $)$ & 0.7 \\
1994 & 1.6 \\
\hline
\end{tabular}

Table 12: Data accumulated per LEP experiment. The counts from the different experiments are equal to within 10-15\%. A centre-of-mass energy scan around the $\mathrm{Z}^{0}$ peak was performed in the years marked "scan". In 1992 and 1994 all data were collected on the top of the $\mathrm{Z}^{0}$ peak.

The approximate numbers of hadronic $\mathrm{Z}^{0}$ decays collected by the LEP experiments are given in table 12. High-resolution microvertex detectors, very important for high precision electroweak measurements in the heavy flavour sector, were installed and commissioned by ALEPH, DELPHI and OPAL in 1990 and 1991, and by L3 in 1993-1994. As a result, 1.5 to 3 million hadronic $\mathrm{Z}^{0}$ decays are available per experiment, with largely uniformly good data quality. Many of the results reviewed in this paper do not yet include the data collected in 1993, and none yet include 
data collected in 1994. Further data will be taken at the $\mathrm{Z}^{0}$ peak in 1995 , before the beam energy is subsequently raised to the $\mathrm{W}$ pair production threshold at LEP-II. Thus a combined total yield of $15-20$ million hadronic $\mathrm{Z}^{0}$ decays will be available by the time LEP-I data-taking is completed.

Extrapolating from the current measurements to the likely final precision of the full LEP-I data set is difficult, because so far only a relatively small fraction of the data have been analysed, and there is plenty of scope for new analysis techniques. If it is assumed that the present analysis methods will remain largely unchanged, however, reasonable estimates can be made.

The measurement of $\Gamma_{\mathrm{b}} / \Gamma_{\text {had }}$ is dominated by double lifetime tagging methods, and can be expected to remain so in future. In the pessimistic case that the ALEPH, OPAL and DELPHI analyses remain unchanged except for the addition of more data, and that L3 is able to make a similar measurement for data taken with the new microvertex detector, the ultimate statistical error on $\Gamma_{\mathrm{b}} / \Gamma_{\text {had }}$ would be around $\pm \mathbf{0 . 0 0 0 7}$. Of the systematic errors listed in table 4 , the track resolution uncertainty can be expected to fall as understanding of tracking performance and problems improve, and the hemisphere correlation error may similarly improve somewhat. The uncertainties from the charm background are mostly not amenable to measurement at LEP, but can be reduced by raising the lifetime tag cut, at the cost of increasing the statistical error. Since only some of the systematic uncertainties are correlated between experiments, an ultimate combined systematic error of approximately \pm 0.0013 can be expected. Inclusion of lepton information from all four experiments should reduce the overall error by $10-15 \%$, following current trends, to give a final total precision on $\Gamma_{\mathrm{b}} / \Gamma_{\text {had }}$ at the level of \pm 0.0013 , a relative precision of approximately $\mathbf{0 . 6 \%}$, equivalent, in the standard model framework, to a top mass change of about $50 \mathrm{GeV}$. It must be stressed that new, more powerful, techniques may allow an improvement on this precision.

Measurements of $\Gamma_{\mathbf{c} \bar{c}} / \Gamma_{\text {had }}$ are already systematics limited. Lepton measurements suffer from large errors due to the modelling of the lepton spectra. Improved understanding of these might help to reduce the errors somewhat. Reconstructed particles, in particular $\mathrm{D}^{*+}$ mesons, are currently the source of the most precise measurement. Such tags are, however, limited by external errors, most notably the knowledge of the fraction of $\mathrm{c}$ quarks which hadronise into a $\mathrm{D}$ meson. This has only been measured at lower energies, with errors typically around $10 \%$. No improvements are to be expected in the future. Also important, though not yet really limiting the precision, is the ability of the detectors to separate D mesons from primary c quarks from those from primary b quarks. This area has more scope for future improvement. Significantly better $\Gamma_{\mathbf{c}} / \Gamma_{\text {had }}$ measurements can therefore only be expected if new measurement techniques are developed. Double tag approaches for charm might provide an answer, as they have done for $\Gamma_{\mathrm{b}} \overline{\mathrm{b}} / \Gamma_{\text {had }}$. However, unlike the situation in the $\mathrm{b}$ sector, the available sample of double tagged charm events is very small. With the full data sets collected by the experiments up until the end of 1994, approximately 3.5 million hadronic $Z^{0}$ decays, and considering all decays into charmed mesons for which results have been presented, samples of around 100 double-tagged events per experiment are expected. This is unlikely even to double before LEP-I data-taking is completed. Such a yield is too small for a precise measurement of $\Gamma_{\mathbf{c} \bar{c}} / \Gamma_{\text {had }}$. Only the development of new, more powerful, charm tagging techniques will allow significant improvements.

The second category of measurements discussed in this review, the forward-backward asymmetries, are for the most part still statistically limited. Very similar arguments as given for the case of $\Gamma_{\mathrm{b}} / \Gamma_{\text {had }}$ are applicable for analyses based on lepton tags. The development of more sophisticated fitting procedures taking into account and simultaneously fitting for a more com- 
plete set of electroweak parameters should allow a further and significant decrease in the final error, both for the $b$ and for the c asymmetry.

Measurements of the charm asymmetry using charm tagged samples will profit significantly from the large increase in statistics that will be available by the time LEP-I stops. The precise final error is rather difficult to predict, but it seems feasible to reach errors similar to the ones obtained using the leptonic sample.

There is scope to complement the measurements discussed in this review by making electroweak measurements in the light quark sector. Very few analyses have been shown so far. Progress in the development of techniques to separate these flavours seems possible, however, and measurements of asymmetries for all three light flavours should be feasible.

Further progress in the determination of the electroweak couplings beyond LEP-I will be possible by combining LEP results with present and future results from SLD. The presence of polarised beams at SLD provides interesting and complementary possibilities to the LEP measurements discussed here.

\section{Conclusion}

Techniques for measuring electroweak heavy quark parameters have matured remarkably in the last few years. A wide range of different tagging techniques have been developed. The partial $\mathrm{Z}^{0}$ decay width to $\mathrm{b} \overline{\mathrm{b}}$ pairs has been particularly precisely measured using double lifetime tags. Combining the published results from the LEP experiments, a value of

$$
\Gamma_{\mathrm{b} \overline{\mathrm{b}}} / \Gamma_{\mathrm{had}}=0.2207 \pm 0.0022
$$

is obtained, where the error includes statistical and systematic effects. The averaging procedure employed takes into account LEP measurements of all the heavy quark electroweak quantities, rather than using the standard model predictions. If the rather precise $\Gamma_{\mathrm{b}} / \Gamma_{\text {had }}$ measurements are interpreted in the framework of the standard model, which predicts $\Gamma_{\mathbf{c} \overline{\mathbf{c}}} / \Gamma_{\text {had }}=0.171$ relatively precisely, an average of

$$
\Gamma_{\mathrm{b}} / \Gamma_{\text {had }}=0.2189 \pm 0.0020
$$

is obtained.

Measurement of the $c \bar{c}$ partial width has also been vigorously pursued. A combined LEP result of

$$
\Gamma_{\mathbf{c} \overline{\mathbf{c}}} / \Gamma_{\text {had }}=0.153 \pm 0.011
$$

is obtained by averaging the published measurements using the combined averaging procedure.

Measurements of the forward-backward production asymmetries of $b$ and $c$ quarks in $\mathrm{Z}^{0}$ decays give information about the effective electroweak mixing angle, $\sin ^{2} \theta_{\mathrm{W}}^{\text {eff }}$. A consistent treatment of the measurements and their errors has been used to derive average values, for $\sqrt{s}=91.26 \mathrm{GeV}$, of:

$$
\begin{aligned}
& A_{\mathrm{FB}}^{\mathrm{b}}=0.0902 \pm 0.0045 \\
& A_{\mathrm{FB}}^{\mathrm{c}}=0.070 \pm 0.012
\end{aligned}
$$


from the published measurements. In the context of the standard model, the b quark asymmetry in particular gives a precise measurement of the effective electroweak mixing angle, $\sin ^{2} \theta_{\mathrm{W}}^{\mathrm{eff}}$ :

$$
\sin ^{2} \theta_{\mathrm{W}}^{\mathrm{eff}}=0.2328 \pm 0.0008
$$

The future prospects for improvements of these measurements are excellent. The asymmetry measurements, in particular, are still statistics limited, and are likely to remain so for the duration of LEP-I data-taking. The measurements of $\Gamma_{\mathrm{b}} \overline{\mathrm{b}} / \Gamma_{\text {had }}$ will also improve with increasing statistics, although effort is required to keep the systematic errors under control. Measurements of $\Gamma_{\mathbf{c}} / \Gamma_{\text {had }}$ are largely systematics limited at present. Further improvement requires the development of new techniques.

\section{Acknowledgements}

The measurements presented here are the work of our colleagues in the four LEP collaborations, too numerous to name. We thank them for producing such elegant and well-documented analyses. The staff of the SL Division at CERN are also to be thanked for their continuing stewardship of the LEP accelerator, the excellent and continually improving performance of which makes these precise measurements possible. The combination of the different results would have been a much more arduous task but for the work of the LEP electroweak working group, and its heavy flavour sub-group, in standardising systematic errors.

Valuable assistance was provided by C.M. Hawkes, P. Mättig, K. Mönig, M. Morii, D.P. Stickland, L. Taylor, R. Tenchini and P.S. Wells in the production of this review. In particular we wish to acknowledge P.S. Wells for the provision of the LEP electroweak heavy flavour averaging program.

We wish to acknowledge the support of our funding bodies: the Bundesministerium für Forschung und Technologie, Germany; the Royal Society; and the Particle Physics and Astronomy Research Council, United Kingdom; as well as support staff at CERN. 


\section{References}

[1] See, for example, D. Schaile, Fortsch.Phys. 42 (1994) 429.

[2] Calculated using the ZFITTER program described in:

D. Bardin et al., CERN-TH 6443/92 (May 1992); Phys. Lett. B255 (1991) 290; Nucl. Phys. B351 (1991) 1; Z. Phys. C44 (1989) 493.

[3] The LEP Collaborations, ALEPH, DELPHI, L3 and OPAL, and the LEP Electroweak Working Group, CERN-PPE/94-187, and references therein.

[4] T. Sjöstrand, PYTHIA 5.6 and JETSET 7.3: Physics and Manual, CERN-TH.6488/92;

T. Sjöstrand and M. Bengtsson, Computer Physics Commun. 43 (1987) 367;

T. Sjöstrand, Computer Physics Commun. 39 (1986) 347.

[5] ALEPH Collab., D. Decamp et al., Nucl. Instrum. Methods A294 (1990) 121;

DELPHI Collab., P. Aarnio et al., Nucl. Instrum. Methods A303 (1991) 233;

L3 Collab., B. Adeva et al., Nucl. Instrum. Methods A289 (1990) 35;

OPAL Collab., K. Ahmet et al., Nucl. Instrum. Methods A305 (1991) 275.

[6] JADE Collab., W. Bartel et al., Z. Phys. C33 (1986) 23;

JADE Collab., S. Bethke et al., Phys. Lett. B213 (1988) 235.

[7] OPAL Collab., R. Akers et al., Z. Phys. C61 (1994) 209.

[8] MARK III collab., D. Coffman et al., Phys. Lett. B263 (1991) 135.

[9] Particle Data Group, L. Montanet et al., Phys. Rev. D50 (1994) 1173.

[10] ALEPH Collab., D. Buskulic et al., Phys. Lett. B313 (1993) 535.

[11] Mark II Collab., R.G. Jacobsen et al., Phys. Rev. Lett. 67 (1991) 3347.

[12] OPAL Collab., P.D. Acton et al., Z. Phys. C60 (1993) 579.

[13] DELPHI Collab., P. Abreu et al., CERN-PPE/94-131.

[14] DELPHI Collab., P. Abreu et al., CERN-PPE/94-161.

[15] OPAL Collab., R. Akers et al., CERN-PPE/94-106.

[16] ALEPH Collab., D. Buskulic et al., CERN-PPE/94-165, submitted to Phys. Lett.;

ALEPH Collab., D. Buskulic et al., Phys. Lett. B298 (1993) 479;

L3 Collab., M. Acciarri et al., Phys. Lett. B332 (1994) 201.

[17] L3 Collab., M. Acciarri et al., CERN-PPE/94-089.

[18] OPAL Collab., R. Akers et al., Z. Phys. C60 (1993) 199.

[19] ALEPH Collab., D. Buskulic et al., Z. Phys. C62 (1994) 179;

ALEPH Collab., D. Buskulic et al., Nucl. Instrum. Methods A346 (1994) 461.

[20] N. Isgur, D. Scora, B. Grinstein and M. Wise, Phys. Rev. D39 (1989) 799. 
[21] G. Altarelli et al., Nucl. Phys. B208 (1982) 365.

[22] CLEO Collab., D. Bortoletto et al., Phys. Rev. D45 (1992) 21.

[23] DELCO Collab., W. Bacino et al., Phys. Rev. Lett. 43 (1979) 1073.

[24] MARK III Collab., R.M. Baltrusaitis et al., Phys. Rev. Lett. 54 (1985) 1976.

[25] ALEPH Collab., D. Buskulic et al., Z. Phys. C62 (1994) 1.

[26] ALEPH Collab., D. Buskulic et al., Phys. Lett. B313 (1993) 549.

[27] OPAL Collab., R. Akers et al., CERN-PPE/94-217.

[28] L3 Collab., O. Adriani et al., Phys. Lett. B307 (1993) 237.

[29] For an introduction to neural networks see: D.E. Rumelhart, G.E. Hinton, R.J. William, in Nature 323 (1986) 533;

most analyses use the program JETNET to implement the algorithms: L. Lonnblad et al., Comp. Phys. Commun. 81 (1994) 185.

[30] DELPHI Collab., P. Abreu et al., Phys. Lett. B295 (1992) 383.

[31] DELPHI Collab., P. Abreu et al., Z. Phys. C59 (1993) 533.

[32] OPAL Collab., R. Akers et al., CERN-PPE/94-206.

[33] ALEPH Collab., D. Buskulic et al., Phys. Lett. B335 (1994) 99.

[34] M.H. Seymour, LU TP 94-7, Lund Univ., May 1994.

[35] L. Lyons, D. Gibaut, P. Clifford, Nucl. Instrum. Methods A270 (1988) 110.

[36] L3 Collab., O. Adriani et al., Phys. Rept. 236 (1993) 1.

[37] DELPHI Collab., P. Abreu et al., CERN-PPE/95-08.

[38] ALEPH Collab., D. Decamp et al., Phys. Lett. B244 (1990) 551.

[39] DELPHI Collab., P. Abreu et al., Phys. Lett. B252 (1990) 140.

[40] OPAL Collab., P.D. Acton et al., Z. Phys. C60 (1993) 19.

[41] L3 Collab., O. Adriani et al., Phys. Lett. B292 (1992) 454.

[42] DELPHI Collab., P. Abreu et al., CERN-PPE/94-194.

[43] OPAL Collab., P.D. Acton et al., Z. Phys. C60 (1993) 601.

[44] I.Bigi and R.Rückl, private communication.

[45] B. Lampe, MPI-Ph/93-74;

A. Djouadi, B. Lampe, P.M. Zerwas, MPI-Ph/94-81, UdeM-GPP-TH-94-09, DESY 94-201.

[46] SLD Collab., K. Abe et al., SLAC-PUB-6607;

SLD Collab., K. Abe et al., SLAC-PUB-6644. 
[47] DELPHI Collab., P. Abreu et al., Z. Phys. C53 (1992) 555;

L3 Collab., O. Adriani et al., Phys. Lett. B301 (1993) 136;

OPAL Collab., P.D. Acton et al., Z. Phys. C58 (1993) 405;

P. Mättig, CERN-PPE/93-87.

[48] L3 Collab., B. Adeva et al., Phys. Lett. B238 (1990) 122;

ALEPH Collab., D. Decamp et al., Phys. Lett. B259 (1991) 377;

DELPHI Collab., P. Abreu et al., Phys. Lett. B277 (1992) 371;

OPAL Collab., P.D. Acton et al., Phys. Lett. B291 (1992) 436.

[49] OPAL Collab., R. Akers et al., Z. Phys. C60 (1993) 397.

[50] DELPHI Collab., P. Abreu et al., CERN PPE/94-219. 